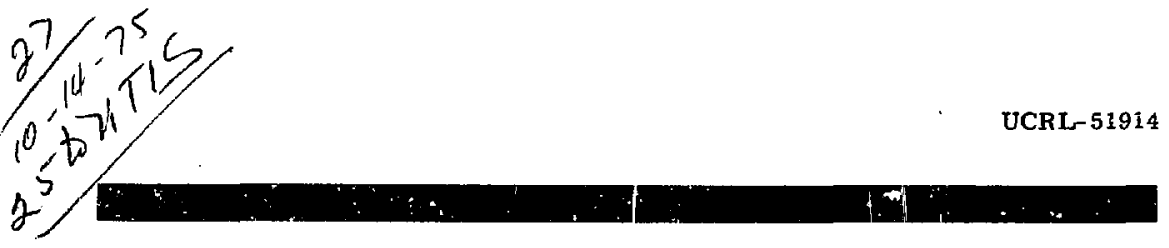

\title{
LASER LIGHT SCATTERING AND ABSORPTION IN DENSE, SPHERICALY SYMMETRIC PLASMAS
}

John Howe Erkkila

(Ph. D. Thesis)

December, 1975

Prepared for U.S. Energy Research \& Development

Administration under contract No. W-i'405-Eng-48
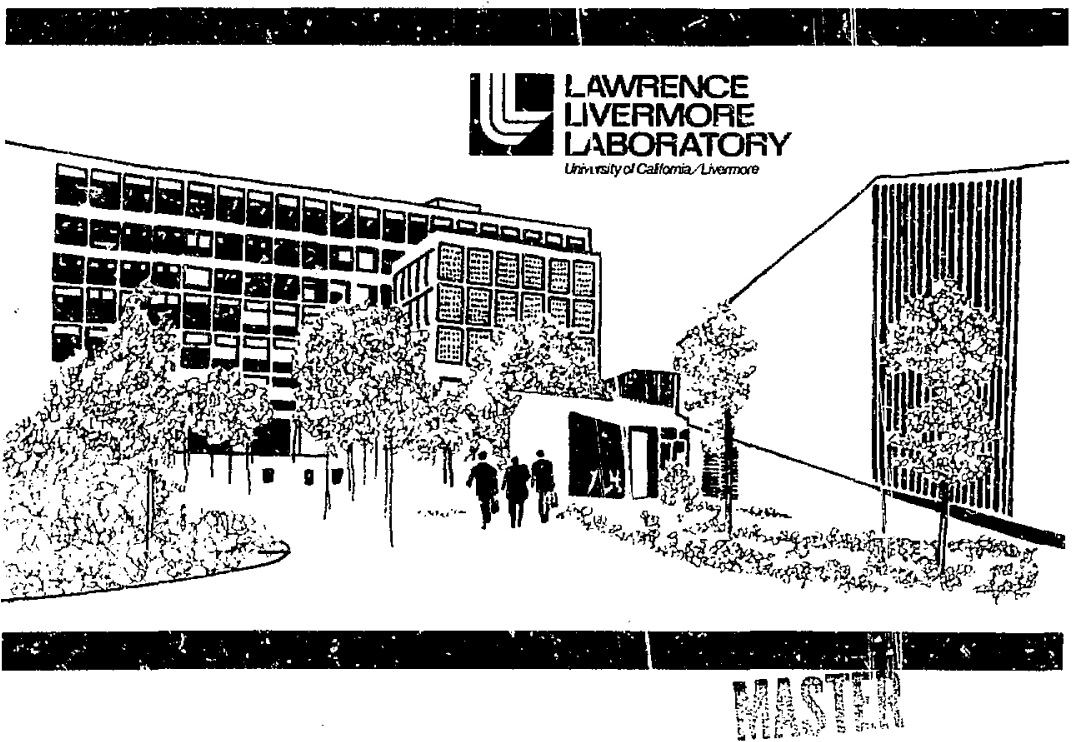
NCT1CE

"This report was prepared as an account of work sponsored by the United States Government. Neither the United States nor the United Staies Energy Rescarch a Devejophenl Adtinistration, nor any of their ampioyets, nor any of their contractors, subconiractois, or theit employecs, makes any wartasty, express or implied. or asumes aity tegal lixbility or responsibility fer the aceuracy. etpleteness or usefulness of any Information, appasatus, proc -as or process disciosed, of represenes that its ise would not infringe privately"ciwned rights."

Printed in the United States of America Available from

National Technical Information Service

U.S. Department of Commerce 5285 Port Royal Road Springfield, Virginia 22151

Price: Printed Cop; $\$$ _*; Micrafiche $\$ 2.25$

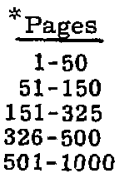

NTIS

Selling Price

$\$ 4.00$

$\$ 5.45$

$\$ 7.60$

$\$ 10.60$

$501-1000$

$\$ 13.60$ 


\section{近 \\ LAWRENCE INERMORE LABOAATORY \\ Lhiversity of Caltomia/Rivemore,Calitomia/\$4550}

\section{UCRL- 51914 \\ LASER LIGHT SCATTERING AND ABSORPTION IN DENSE, SPHERICALLY SYMMETRIC PLASMAS}

John Howe Erkkila

(Ph. D, Thesis)

December, 1975

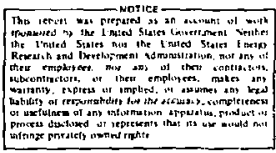


John Howe Erkkila

December 1975

Applied Sctence

\author{
Laser Light Scattering and Absorption in \\ Dense, Spherically Symmetric Plasmas
}

\begin{abstract}
A computational study of the interaction of a focused laser beam with a dense, spherically symmetric plasma is undertaken. The plasma is treated using linearized fluid equations for the electrons with the ions assumed inmobile; the electromagnetic field is determined from strady state solutions to Maxwell's equations in spherical coordinates. Energy dissipation in the plasma occurs as a result of inverse bremsstrahlung collisions and resonance absorption. The scattered field distribution and the spatial variation of the fields and energy deposition within the plasma are found for various laser-plasma configurations and the applicabilfty of the theory as a function of plasma temperature and laser power is determined with a self-consistent analysis.
\end{abstract}




\section{Table of Contents}

Page

Abstract ......................... i i

Table of Contents................. iv

Chapter I: Introduction ............... I

Chapter II: Focused Electrriagnetic Beams in Spherical Geometry 4

A: Introduction ................ 4

B: Debye Potential Functions. . . . . . . . . 5

C: Incident Potential Functions in Focal Volume . . . . . 7

D: Power in Incident Beam ................ 14

E: Results................... 16

F: sumnary. .................. 18

Chapter III: Electromagnetic Waves in a Dense, Spherically Symmetric Plasma ........... 27

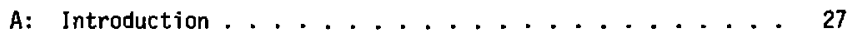

B: Equations.................... 28

c: Solutions in Spherical Coordinates ........... 31

D: Numerical Solutions. ............... 40

E: Resutts. ................... 43

F: Sumary. ................... 47

Chapter IV: Laser Light Scattering and Absorption . . . . . 57

A: Introduction ................ 57

B: Total Absorption and Scattered Light Distribution. . . . 58

C: Fields Inside the Plasma ............. 65

D: Self-Consistent Analysis of Approximations ....... 69

Chapter V: Summary and Conclusion ........... 111 
Page

Acknowledgments .................... 115

Appendix 1: Diffraction of Light by Circular and Annular

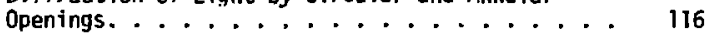

Appendix 11: Vector Fields in the Focal Volume of a Converging

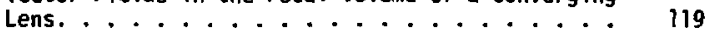

Appendix III: Spherical Bessel Functicns. . . . . . . . 124

Appendix IV: firy Function Solutions ........... 127

Appendix V: Absorption with Linear Gradients. ........ 130

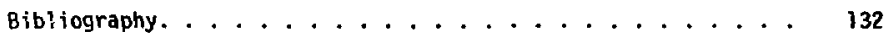




\section{INTRODUCTION!}

Tne heating of a dense plasm:a by a laser is now a well-known proposal for the generation of power through controlled themonuclear reactions. The efficiency and uniformity of the absorption of laser light by the plasma is clearly a very impoitant asfect of this proposal (NUCKOLLS, HOOD, THIESSEN, AND ZIMMERMAN (1972)). Energy transfer from a laser to an inhomogeneous plasma occurs as a result of Joule heating in the underdense plasma atmosphere surroundinn the central core, by means of inverse bremsstrahlung collisions. As the temperature of the plasma rises, the frequency of the collisions decreases, however, and the las s light, which is reflected at the interior surface of the plasma where the laser frequency is equal to the nlasma frequency (called the critical surface), deposits iittle energy by means of classical collisions in a hot plasma. Substantial ieating can still take place. even in the absence of classical collisions, by means of yarious processes which transfer energy from the electromagnetic viaves to plasma waves, which in turn, heat the plasma. One of these processes, called resonance absorption, occurs in inhomogeneous plasmas when an electromagnetic wave, polarized such that the electric field vector has a component in the direction of the inhonogeniety, reaches the critica! surface, where strong coupling to plasma waves occurs. A sigrificant fraction of the electromagnetic energy can be converted to plasma waves in the interaction, but in spherical plasmas the subsequent heating can be highly nonuni form. 
Resonance absorption, by virtue of its polarization dependence, is correctly treated only by solving the full set of Mandell's equations. Previous studies have considered these solutions in Cartesian geometry, where plane electromagnetic waves are incident onto infinite, inhomogeneous plasma slabs. However, the proposed laser fasion systems and many fusion experiments are basically spherical in design, w'th small, dense, spherical plasmas being irradiated by focused laser beams.

To study classical and resonance absorotion in spherical geometry, we derive the boundary conditions appropriate to a focused laser hean, and for these boundary values, determine the steady state solutions to Maxwell's equations in spherical coordinates using linearized fluid equations to cescribe tise plasma. Although the energy depositicn is inherently a nonlinear process, both resonance and classical collisional absorption can be treated with linear equations using these approxi.. mations. The solutions we use require that the olasma remain spherically symmetric and as a consequence, large asymmetric modifications of the density gradient which result from asymnetric heating cannot be considered. Futhermore, as we employ a fluid model for the electrons, kinetic theory effects in the heating of the plasma are not treated, and all ion motion is ignored.

However, the effects of diffraction and light polarization are included and we can determirie accurately both the resonance and classical collisional absorption, and the scattered and refracted light for quite arbitrary laser beams and arbitrary radial density profiles for a wide range of plasma sizes and temperatures. 
The remainder of this report is divided into three major sections, followed by a summary and several appendices:

Chapter II is concerned with free space solutions to Haxwell's equations in the focal volume of an arbitrary lens system, by means of which the boundary conditions needed to solye the plasma interaction problem are found and are related to the incident beam and focusing lens parameters.

In Chapter 111 we discuss the sclutions to Maxwe 17 's equations in a dense, spherically symetric plasma, deriving the equations and the numerical schemes by which we calculate the electromaqnetic fields inside and outside the plasma.

Chapter IV combines the results of the previous two chapters, and discusses the scattering and absorption of laser beams.

We surmarize the conciusions drawn from this study in Chapter $V$.

The appendices are used to illustrate in more detai? mathematical or numerical results gertaining to the techniques employed in the anilysis which otherwise do not contribute to the major thene of the report. 


\section{I1. FOCUSED ELECTROMAGNETIC BEAMS IR SFHERICAL GEOMETRY}

\section{A. Introduction}

The situation we are analyzing is this: a linearly polarized laser beam propagates through an ideal, converging Tens, which converts the plane phase fronts of the beam into spherically converging fronts whose associated geometrical rays intersect, at the focus of the lens. We are to determine the interaction of the focused laser light with an object in the focal volume. To accomplish this, we must find the incident field on the surface of the object, and solve the boundary value problem appropriate to that field. The problem addressed in this chapter is the determination of the incident field in the focal volume in the absence of a plasma; this result is then used as the incident wave for the scattering problems discussed in subsequent chapters.

We do this in the following steps. First, because the equations describing the interaction with the plasma are solved in Chapter III in terms of potentia? functions, we must specify the boundary conditions in terms of these functions, so we determine the relationships between the incident potentials and the incident fields, which are also unknown at this time. Next we relate the field distribution in the incident laser beam to the field components in the focal volume of the lens. Finally we specify the field distribution in the focal volume in a form from which the potential functions needed for the plasma scattering calculations can be extracted. 
Our technique for solving the wave equation requires that the incident wave be defined with respect to an origin at the center of spherical symmetry, that is, the center of the plasma and not the focus of the lens. Therefore it will be necessary to transform: the incident wave from the natural coordinate system for the laser light (the phase fronts are spherical with respect to the geometrical focus of the lens) to arbitrary positions. For simplicity, we will consider only transforms which retain axial symmetry, that is, we will consider only the translation of the target away from or toward the laser along the axis of the laser.

\section{B. Debye Potentia? Functions}

A vector theory of diffraction capable of describing the electromagnetic fields in the focal volume of a lens was first given by Luneberg, see LUNEBERG (1966), p. 311 , and was rederived in a form suitable for the numerical evaluation of the focal volume fields by WOLF (1959), and RICHARDS and WOLF (1959) (henceforth referred to as RW). The RW results express the Cartesian components of the electromagnetic field in the fucal volume in terms of a surface integral over the intersection of a focused beam and a sphere centered on the geometrical focus of the lens. Anticipating the results of Chapter III, we look for a method of expanding the focal volume fields in terms of the solutions of the vector wave equation in free space and in spherical coordinates (a product of the spherica $i$ Bessel functions and 
the spherical harmonics). The most straightforward means of making this expansion does not use the RW results, but employs a derivation similar to that of RW.

Our solutions of the electromagnetic diffraction problem are based on the Mie theory of vector diffraction and employ the Debye potentia] functions. Details and references are given in BORN and WOLF (1975) pp. 633-647 (henceforth referred to as BW). We will apply the boundary conditions appropriate to the laser and lens parameters using the method developed by RW to the vector solutions of Mie theory.

From Mie theory it follows that the electromagnetic fields ir all space can be determined from two potential functions, called $x$ and $\psi$ (BW use notation ${ }^{e_{\text {II }} \text { and }} m_{\Pi}$ where $e_{\pi}=i k x$ and $m_{\Pi}=i k \psi$ ), which are solutions to the scalar wave equations

$$
\begin{aligned}
& \nabla^{2} x+k^{2} x=0 \\
& \nabla^{2} \psi+k^{2} \psi=0
\end{aligned}
$$

The electromagnetic fields are given by

$$
\begin{aligned}
& E_{r}=\frac{1}{i k}\left(\frac{\partial^{2}}{\partial r^{2}}+k^{2}\right) r_{\chi} \\
& E_{\epsilon}=\frac{1}{i k r} \frac{\partial}{\partial \theta} \frac{\partial}{\partial r} r_{\chi}+\frac{1}{\sin \theta} \frac{\partial}{\partial \phi} \psi \\
& E_{\phi}=\frac{1}{i k r} \frac{1}{\sin \theta} \frac{\partial}{\partial \phi} \frac{\partial}{\partial r} r \chi-\frac{\partial}{\partial \theta} \psi \\
& B_{r}=\frac{l}{i k}\left(\frac{\partial^{2}}{\partial r^{2}}+k^{2}\right) r \chi
\end{aligned}
$$




$$
\begin{aligned}
& B_{\theta}=-\frac{1}{\sin \theta} \frac{\partial}{\partial \phi} x+\frac{1}{i k r} \frac{\partial}{\partial \theta} \frac{\partial}{\partial r} r \psi \\
& B_{\phi}=\frac{\partial X}{\partial \theta}+\frac{1}{i k r} \frac{1}{\sin \theta} \frac{\partial}{\partial \phi} \frac{\partial}{\partial r} r \psi
\end{aligned}
$$

As solutions of the scalar Helmholtz equation which are regular at the origin, $x$ and $\psi$ can be expanded in the series:

$$
\begin{aligned}
x(r, \theta, \phi)= & \sum_{\ell=0}^{\infty} j_{\ell}(k r)\left[a_{\ell} p_{\ell}(\cos \theta)\right. \\
& \left.+\sum_{m=1}^{\ell}\left(a_{\ell}^{m} \cos m \phi+b_{\ell}^{m} \sin m\right) P_{\ell}^{m}(\cos \theta)\right] \quad 2.3 . a \\
\psi(r, \theta, \phi)= & \sum_{\ell=0}^{\infty} j_{\ell}(k r)\left[c_{\ell} P_{\ell}(\cos \theta)\right. \\
& \left.+\sum_{m=1}^{\ell}\left(c_{\ell}^{m} \cos m \phi+d_{\ell}^{m} \sin m \phi\right) P_{\ell}^{m}(\cos \theta)\right]
\end{aligned}
$$

where $j_{\ell}(k r)$ is the spherical Besse? function and $P_{\ell}^{m}(\cos \theta)$ is the Legendre polynomial.

The coeficients in the expansions of the Debye potential functions are determined by the boundary conditions, which are in turn, a function of the laser and lens parameters.

II. C. Incident Potential Functions in Focal Volume

Consider the laser-lens-focal volume schematic in Figure II. 1 
(we use largely the notation of RW and follow the line of their derivation closely).

$e_{-0}$ and $\underline{b}_{0}$ are the electric and magnetic field vectors incident onto the lens.

$\underline{e}_{1}$ and $\underline{b}_{7}$ are the electric and magnetic field vectors in the focal volume.

$g_{6}$ and $g_{1}$ are vectors perpendicular to the rays $A B$ and $C O$ which are in the plane of the rays and the axis of symmetry NO.

$e_{x}, e_{y}, e_{z}$ is a Cartesian coordinate system situated on the lens focus.

$\underline{e}_{\gamma}, \underline{e}_{\theta}, \underline{e}_{\phi}$ is a spherical coordinate system at the same focus. For an electric field polarized in the $x$ direction,

$$
\begin{aligned}
& \underline{e}_{0}=a(r) \underline{e}_{x} \\
& \underline{b}_{0}=a(r) \underline{e}_{y}
\end{aligned}
$$

where $a(r)$ is an arbitrary profile chosen for the incident beam. At this time we are restricting ourselves to axisymmetric incident laser beams, by allowing for no azimuthal dependence of the 1 ight.

We want to decermine

$$
\begin{aligned}
& \underline{e}_{1}=a(\theta)\left[\gamma \underline{e}_{r}+\alpha \underline{e}_{\theta}+\beta \underline{e}_{\phi}\right] \\
& \underline{b}_{1}=\underline{s} \times \underline{e}_{1}
\end{aligned}
$$

Because $\underline{e}_{1}$ is perpendicular to $\underline{s}$, a radial vector, $\gamma=0$. Two additional factors affect $\underline{e}_{1}$ and $\underline{b}_{1}$. First, according to geometrical 
optics, the angle: between $\underline{g}_{0}$ and $\underline{e}_{0}$ and $\underline{g}_{7}$ and $\underline{e}_{7}$, and the angles between $g_{0}$ and $\underline{b}_{0}$ and $g_{1}$ and $\underline{e}_{1}$ must not be changed by the lens (RW, p. 362).

Therefore

$$
g_{1} \cdot \underline{e}_{1}=a(r) g_{0} \cdot \underline{e}_{x}
$$

and

$$
\left(\underline{g}_{1} \times \underline{s}\right) \cdot \underline{e}_{1}=a(r) g_{0} \cdot \underline{e}_{y}
$$

But

$$
g_{0}=\cos \phi \underline{e}_{x}+\sin \phi \underline{e}_{y}
$$

so

$$
g_{1} \cdot \underline{e}_{1}=a\langle r\rangle \cos \phi
$$

$$
\left(\underline{g}_{1} \times \underline{s}\right) \cdot \underline{e}_{1}=a(r) \sin \phi
$$

and

$$
\begin{aligned}
& g_{1}=-\underline{e}_{\theta} \\
& g_{1} \times \underline{s}=-e_{\phi}
\end{aligned}
$$

so

$$
\begin{aligned}
& \underline{e}_{1}=-a(r)\left[\cos \phi \underline{e}_{\theta}+\sin \phi e_{\phi}\right] \\
& \underline{b}_{1}=-a(r)\left[\sin \phi \underline{e}_{\theta}-\cos \phi e_{\phi}\right]
\end{aligned}
$$

Secondly, the type of projection affects the amplitude of $\underline{e}_{1}$ and $\underline{b}_{1}$ because the incident rays are not necessarily uniformly spread over the sphere in the focal volume.

We shall consider here the aplanati: projection $r=\sin \theta$, for which the amplitude of a field component changes by the factor $\sqrt{\cos \theta}$ when passing through the lens. 
Then, for an aplanatic lens

$$
\begin{aligned}
& \alpha=-\sqrt{\cos \theta} \cos \phi \\
& \beta=-\sqrt{\cos \theta} \sin \phi
\end{aligned}
$$

and, making the change of argument, $a(r)=a(r(\theta))=a(\theta)$, we have

$$
\begin{aligned}
& \underline{e}_{1}=-a(\theta) \sqrt{\cos \theta}\left[\cos \phi \underline{e}_{\theta}+\sin \phi e_{\phi}\right] \\
& \underline{b}_{1}=-a(\theta) \sqrt{\cos \theta}\left[\sin \phi \underline{e}_{\theta}-\cos \phi \underline{e}_{\phi}\right]
\end{aligned}
$$

Thus far we have determined the spherical components of the field vectors on the surface of a sphere at a radius $R$ in the focal volume. In order to solve the focusing problem we must determine the Debye potential functions there.

From equation 2.2 we see that the Debye potentials are uniquely deterrilined by the radial components of $\underline{e}_{7}$ and $\underline{b}_{1}$, and from equation 2.8 these appear to be identically zero! We have used geometrical optics to this paint, however, and have ignored the transverse variations in the beam. In fact, the radial field components are not zero, and can readily be obtained from Maxwell's equations:

$$
\begin{aligned}
& \underline{r} \cdot \underline{\nabla} \times \underline{E}=i k r B_{r} \\
& \underline{r} \cdot \underline{\nabla} \times \underline{B}=-i k r E_{r}
\end{aligned}
$$

Then, using

$$
S(\theta)=\ldots ; \theta) \sqrt{\cos \theta}
$$




$$
\begin{aligned}
& \underline{r} \cdot \underline{\nabla} \times \underline{E}=-\frac{S(\theta)}{\sin \theta} \sin \phi\left(1-\cos \theta+\frac{\sin \theta}{S(\theta)} \frac{\partial S}{\partial \theta}\right) \\
& \underline{r} \cdot \underline{\nabla} \times \underline{B} \quad \frac{S(\theta)}{\sin \theta} \cos \phi\left(1-\cos \theta+\frac{\sin \theta}{S(\theta)} \frac{\partial S}{\partial \theta}\right)
\end{aligned}
$$

of course the radial components are smaller than the transverse components by the factor $k R$.

Writing the expressions in the form:

$$
\begin{aligned}
& \underline{r} \cdot \underline{\nabla} \times \underline{\underline{E}}^{i n c}=-\sin \phi W(\theta) \\
& \underline{r} \cdot \underline{\nabla} \times \underline{\underline{B}}^{i n c}=\cos \phi W(\theta) \\
& 2.12 . \mathrm{b} \\
& W(\theta)=\frac{a(\theta) \sqrt{\cos \theta}}{\sin \theta}\left[1-\cos \theta+\sin \theta \frac{\partial}{\partial \theta} \ln (\sqrt{\cos \theta} a(\theta))\right] 2.12 . c
\end{aligned}
$$

where we use the superscript inc to identify the fields as the incident ones in the scattering problem.

Because these fields are purely incoming with respect to the lens focus, the potentials can be expanded as a series of incoming spherical Hankel functions, $h_{\ell}^{(2)}(k r)$. Thus

$$
\begin{aligned}
x^{i n c}(r, \theta, \phi) & =\sum_{\ell=0}^{\infty} h_{\ell}^{(2)}(k r)\left[a_{\ell} p_{\ell}(\cos \theta)\right. \\
& \left.+\sum_{m=1}^{\ell}\left(a_{\ell}^{2 m} \cos m \phi+\hat{b}_{\ell}^{m} \sin m \phi\right) P_{\ell}^{m}(\cos \theta)\right]
\end{aligned}
$$




$$
\begin{aligned}
\psi^{\text {inc }}(r, \theta, \phi) & =\sum_{\ell=0}^{\infty} n_{\ell}^{(2)}(k r)\left[c_{\ell}^{2} p_{\ell}(\cos \theta)\right. \\
& \left.+\sum_{m=1}^{\ell}\left(\hat{c}_{\ell}^{m} \cos m \phi+\partial_{\ell}^{m} \sin m \phi\right) p_{\ell}^{m}(\cos \theta)\right]
\end{aligned}
$$

and at $r=R, x$ and $\psi$ can be related to $E_{r}$ and $B_{r}$ using equations 2.2.a and 2.2.d.

Because $x$ and $\psi$ must remain finite at $r=0$, the coeficients with the tilde's can be simply related to those without, this being

$$
2^{2} a_{\ell}^{m}=a_{\ell}^{m}
$$

and similariy for coefficients b, $c$, and d. From equations 2.2, 2.9, 2.11 and 2.13,

$$
\begin{aligned}
E_{r}^{i n c}=\frac{1}{i k r} & \sum_{\ell=1}^{\infty} \ell(\ell+1) h_{\ell}^{(2)}(k r)\left[\tilde{a}_{\ell} P_{\ell}(\cos \theta)\right. \\
& \left.+\sum_{m=1}^{\ell}\left(a_{\ell}^{2 m} \cos m \phi+\tilde{\theta}_{\ell}^{m} \sin m \phi\right) P_{\ell}^{m}(\cos 0)\right] \quad \text { 2.15.a }
\end{aligned}
$$

and

$$
\begin{aligned}
E_{r}^{i n c}= & -\frac{1}{i k r} \cos \phi W(\theta) \\
B_{r}^{i n c}= & \frac{1}{i k r} \sum_{\ell=1}^{\infty} l(l+1) h_{l}^{(2)}(k r) \tilde{c}_{\ell} p_{\ell}(\cos \theta) \\
& \left.+\sum_{n=1}^{\ell}\left(c_{l}^{m} \cos m+\gamma_{l}^{m} \sin m \phi\right) P_{\ell}^{m}(\cos \theta)\right]
\end{aligned}
$$


and

$$
B_{r}^{\text {inc }}=-\frac{1}{i k r} \sin \phi W(\theta) .
$$

Then, expanding $\cos \phi W(\theta)$ and $\sin \phi W(\theta)$ in a series of spherical harmonics at $r=R$, and equating coefficients, we find

$$
\begin{aligned}
& \hat{b}_{\ell}^{m}=z_{\ell}^{m}=0 \text { for all } \ell, m \\
& a_{\ell}^{m m}=u_{\ell}^{m}=0 \text { for } m \neq 1 \\
& \tilde{a}_{\ell}^{l}=\tilde{d}_{\ell}^{1}=-\frac{2 \ell+1}{2 \ell^{2}(l+1)^{2}} \frac{\int_{0}^{\pi} W(\theta) P_{\ell}^{1}(\cos \theta) \sin \theta d \theta}{h_{\ell}^{(2)}(k R)}
\end{aligned}
$$

Thus the problem is solved since $W(\theta)$ is known (equation 2.12.c), and $x^{\text {inc }}$ and $\psi^{\text {inc }}$ are given in equation 2.13. In the implementation of this technique, this integral (2.16.c) is solved numerically for all l's used in the series expansion of the Debye potentidl functions. Axial symmetry of the beam leads to the vanishing of all coefficients for which $m \neq 1$, but it is mainly for convenience that we consider such laser sources. The projection for arbitrary beams involves the numerical evaluation of two dimensional integrals and $\ell \max / 2$ times as many evaluations.

Modifications to include phase aberrations on the laser beam are straightforward, $a(\theta)$, as introduced in equation 2.4 , is treated as a complex number, and the prescribed deviation of the phase from some reference is carried through the calculations of $S(\theta), W(\theta)$, and the coefficients $a_{\ell}^{l}$ and $d_{R}^{l}$ (which are already, in general, complex).

The expansion of the Debye potential ahout a shifted origin can also be incorporated as an adjustment to aij), as in Figure II.2. 
11. D. Power in Incident Beam

As a final step in the theoretical development of a vector diffraction theory using Debye potentials, we will derive in expression for the Poynting's vector and power in the incident bears, in terms of the expansion coefficients of $\chi$ and $\psi$.

Poynting's vector for harmonic electromagnetic firslds is given by the expression:

$$
\left.S_{r}=\frac{C}{8 \pi} R_{e}(r) \times \underline{B}^{*}\right)
$$

Using equations 2.2, the radial component of $S i$;

$$
\begin{aligned}
& S_{r}=\frac{c}{8 \pi} R_{e}\left[\left(\frac{1}{i k r} \frac{\partial}{\partial r} \frac{\partial}{\partial \theta} r \chi \frac{\partial}{\partial \theta} X^{\star}+\frac{1}{i k r} \frac{1}{\sin ^{2} \theta} \frac{\partial}{\partial r} \frac{\partial}{\partial \phi} r_{\chi} \frac{\partial}{\partial \phi} \chi^{\star}\right.\right. \\
& -\frac{l}{i k r} \frac{l}{\sin ^{2} \theta} \frac{\partial}{\partial \bar{\phi}} \psi \frac{\partial}{\partial r} \frac{\partial}{\partial \phi} r^{*}-\frac{l}{i k r} \frac{\partial \psi}{\partial \theta} \frac{\partial}{\partial r} \frac{\partial}{\partial \theta} r_{\psi^{*}} \\
& +\frac{1}{\sin \theta}\left(\frac{\partial \psi}{\partial \phi} \frac{\partial}{\partial \theta} x^{*}-\frac{\partial}{\partial \theta} \psi \frac{\partial}{\partial \phi} x^{*}\right) ; \frac{1}{k^{2} r^{2}} \frac{1}{\sin \theta}-\left(\frac{\partial}{\partial r} \frac{\partial}{\partial \theta} r x \frac{\partial}{\partial r} \frac{\partial}{\partial \phi} r \psi^{*}\right. \\
& \left.\left.-\frac{\partial}{\partial r} \frac{\partial}{\partial \phi} r_{X} \frac{\partial}{\partial r} \frac{\partial}{\partial \Phi} r \psi^{\star}\right)\right] \quad 2.18
\end{aligned}
$$

Then, substituting equations 2.13 and integrating over the sphere at $r=R$

$$
\begin{array}{r}
\int_{R} \underline{S} \cdot d A=\frac{c}{B} R^{2} R_{e}\left[\frac { 1 } { i k r } \sum _ { \ell } a _ { \ell } a _ { l } ^ { * } \left\langle\frac{\partial}{\partial r} r h_{l}^{(2)_{h}(2)^{*}}\right.\right. \\
\left.-h_{\ell}^{(2)} \frac{\partial}{\partial r} r h_{\ell}^{(2)^{*}}\right)
\end{array}
$$




$$
\begin{aligned}
& x\left(\int_{0}^{\pi} d \theta\left\{\sin \theta\left(\frac{d P_{\ell}^{1}}{d \theta}\right)^{2}+\frac{!}{\sin \theta}\left(P_{l}^{1}\right)^{2}\right\}\right) \\
& \left\{\left(\sum_{\ell, n} a_{\ell}^{*} a_{n} h_{l}^{(2)^{*}} h_{n}^{(2)}+\frac{1}{k^{2} r^{2}}\left\{a_{\ell} \frac{\partial}{\partial r} r h_{\ell}^{(2)} a_{n}^{*} \frac{\partial}{\partial r} r h_{n}^{(2) *}\right\}\right)\right. \\
& \left.x\left(\int_{0}^{\pi} d \theta\left\{P_{n}^{1} \frac{d P^{1}}{d \theta^{2}}+P_{2}^{1} \frac{d P^{1}}{d \theta^{1}}\right\}\right)\right\} \text {. }
\end{aligned}
$$

The second term, which results from couplin! between terms in the $x$ and $t_{1}$ expansion, vanishes because of the integration over $\theta$, and the angular portion of the first term can be integrated, giving:

$$
\begin{aligned}
& \int_{R} \underline{S} \cdot d A=\frac{c R^{2}}{4} R_{e} / \sum_{l} \frac{\ell^{2}(l+1)^{2}}{2 \ell+1} \frac{a_{\ell^{a}}{ }^{*}}{i k R}\left(\frac{\partial}{\partial r}\left(r \dot{n}_{l}^{(2)}\right) n_{l}^{(2) *}\right. \\
& -h_{\ell}^{\left.\left.(2) \frac{\partial}{\partial r}\left(r h_{\ell}^{(2)^{*}}\right)\right)\right] .}
\end{aligned}
$$

For $k R \gg>1$, the asyintotic form of the spherical Hankel function can be used, giving:

$$
\begin{aligned}
\int_{R} \underline{S} \cdot \underline{d A} & =\frac{c R^{2}}{k R} \sum_{\ell} \frac{\ell^{2}(\ell+1)^{2}}{2 \ell+1} a_{\ell} a_{\ell}{ }^{*} \\
& =\frac{c R^{2}}{A(k h)^{3}} \sum_{\rho} \frac{2 \ell+1}{\ell_{\ell}^{2}(\ell+1)^{2}} I_{\ell} I_{\ell}
\end{aligned}
$$

where

$$
I_{\ell}=\int_{0}^{\pi} W(\theta) P_{\ell}^{I}(\cos \theta) \sin 0 d \theta
$$

from equation 2.16.c. 
Identifying the integrated Poynting vector with the power in the beam, we can identify, by means of equation 2.21 , the $\Omega$ dependence of tric power, i.e.,

$$
\begin{aligned}
& P=\sum_{\ell} P_{\ell} \\
& P_{\ell}=\frac{C R^{2}}{4(k R)^{3}} \frac{2 \ell+1}{\ell^{2}(\ell+1)^{2}} I_{\ell} I_{\ell}^{*} .
\end{aligned}
$$

The significance of this dependence is made clear in the following chapters where the $\ell$ dependence of the resonance absorption and scattering is determined.

II. E. Resuits

We will consider $P_{\ell}$ (Equation 2.22\} as a function of the following parameters:

(1) $f \#$ of the lens, where, fram Figure II.1.

$$
f \#=\frac{1}{2 \tan e_{m}}
$$

$\theta_{m}$ is the value of $\theta$ corresponding to $r=r_{0}$, when the incident electric field amplitude is given by $a(r)=\exp \left\{-\left(r / r_{0}\right)^{n}\right\}$

(2) $p$, target translation relative to the geometrical focus of lens, $\rho$ is positive in a direction toward the laser.

(3) $w$, number of waves of spherical aberration resulting from nonlinear effects in the laser. As a result of the nonlinear index of refraction of the laser medium, the more intense center of the laser 
beam has a slightly increased refractive index, as compared to the low intensity edge of the beam, and as a consequence, the on-axis phase is delayed relative to the edge (GLAZE, SPECK and HUNT (1975)). $W$ is the number of wavelengths delay between the center and the edge of the jeam. The phase variation across the beam is $\phi=w I(v) / I(0)$, where $I(r)$ is the beam intensity profile. $W$ is small early in the laser pulse when the nonlinear effects are weak, but at the peak of an intense laser pulse, w may be as large as two or three waves.

For the remainder of this report we will use $N=5$ to define the electric field in the laser beam. $I(\theta)$ is plotted in Figure 11.3 for $f \# 0.5$, where $I=a(\theta) a^{\star}(\theta)$.

In Figures $I I .4$ and $I I .5$ we plot $P_{\ell}$ versus \& for variations of $\rho$. Notice that both positive and negative translations have identical multipole moments, and that they are fairly flat over a width of $\ell$ 's which becomes large as the translation is made large. Structure exists on the spectral plots for $\rho=0$, which is a result of the nigher hamionics of $\theta_{m}$. This structure quickly washes out when beam aberrations are included or when translation, away from 0 are made.

In Figure II.6 we plot $P_{\ell}$ versus $\ell$ for variations of $f f$. Here we notice that the higher f\#'s, which have the incident beam squeezed into a narrower angular range, for $p=0$, have a broader multipole spectrum. On the other hand, for the higher $f$ 's a greater translation is required to cause a shift in the multipole spectrum. This occurs because the waist of a high $f \#$ beam is long and slender, and a large translation is necessary to observe changes in its characteristics. 
In Figures II.7 and II.8 we plot $P_{\ell}$ versus $\ell$ for variations of $w$, $\mathrm{f \#}$ and $p$. Here we see that the effect of spherical phase aberrations is to remove the symmetry of the translation about $\rho=0$ and to generally broaden the multipole spectrum.

\section{F. Surmary}

We have developed an alternative method for calculating the electromagnetic field in the focal volume of a converging lens using the Debye potential functions, and we have derived the expressions which relate the coefficients in the spherical harmonic expansion of the Debye functions to the parameters of the laser and the focusing lens. Computer implementation of this procedure provides a very fast method for calculating the vector fields in the focal volume of a converging lens, although the main objective in its development is to specify the boundary conditions needed in the plasma interaction problem, which is to be discussed in the following two chapters. For simplicity we have assumed that the laser beam is axisynnetric. Implicitly we have made the Kirchhoff approximation in our boundary conditions at the output of the lens, and we have ignored diffraction, scattering, and absorption in the lens.

Numerical solution of some exact diffraction problems are given in Appendix 1, as a means of verifying the computer solutions. In Appendix 2 we compare vector diffraction calculations with those of RICHARDS and WOLF (1959). 


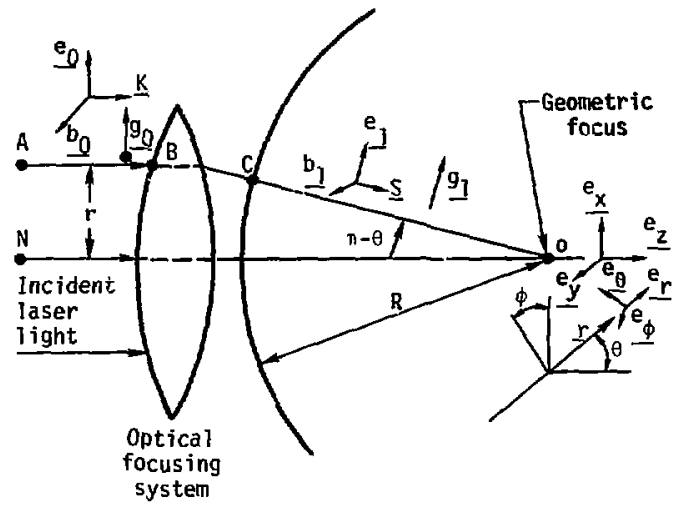

Figure II.T Coordinate systems and geometrical definitions used in determining the effect of an optica? focusing system on the laser beam. 


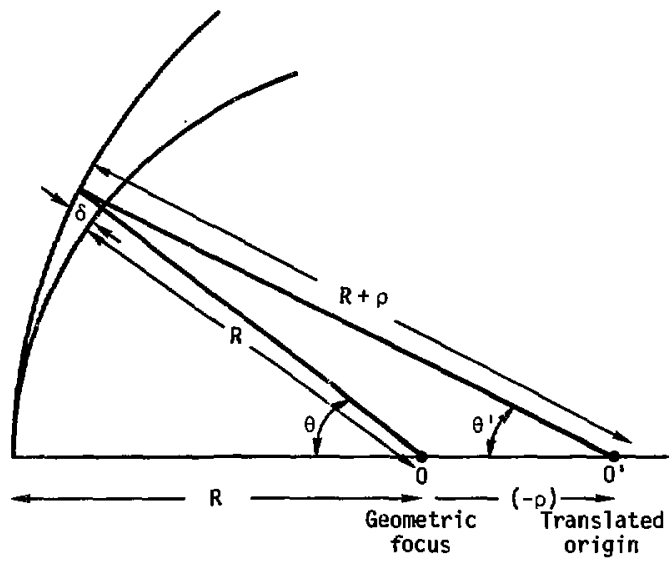

$\rho$ is positive to the left, toward the laser.

Phase difference of wave with respect to origins 0 and $0^{\circ}$ is $\Delta \phi=k(\delta-\rho) \approx-k \rho \cos \theta$

Fignire II.2 Effect of translation on the phase of a spherically converging wave. 


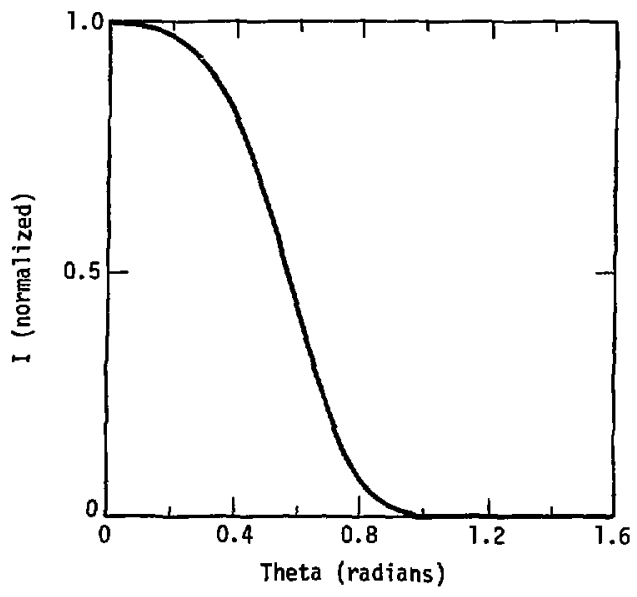

Figure II.3 Incident beam angular intensity profile. $f \#=0.5$. 


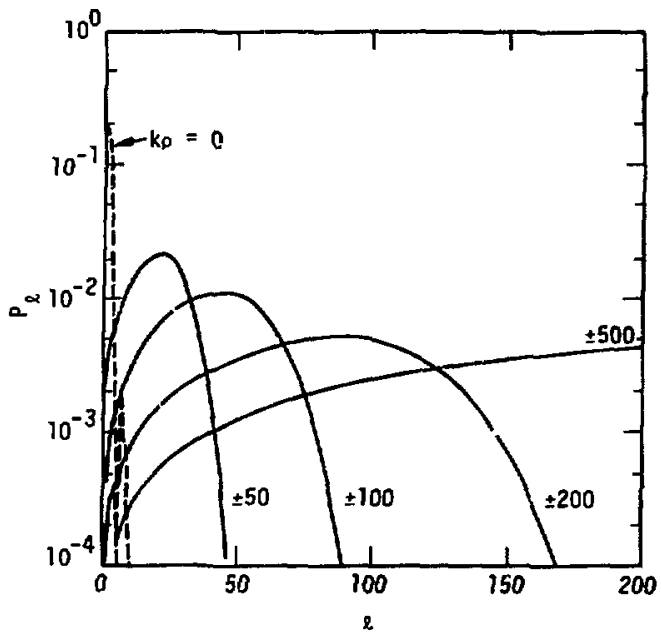

Figure II.4 Multipole spectra of a focused laser bean. $f *=0.5, \mathrm{k \rho}$ from $n$ to \pm 500 . 


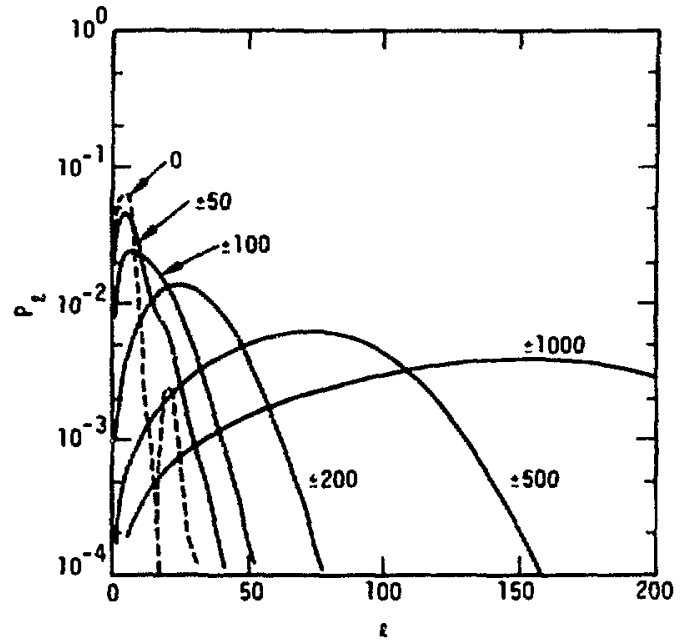

Figure 11.5 Multipole spectra of a focused laser beam. $f_{f}=2.0, k n$ from 0 to $=1000$ 


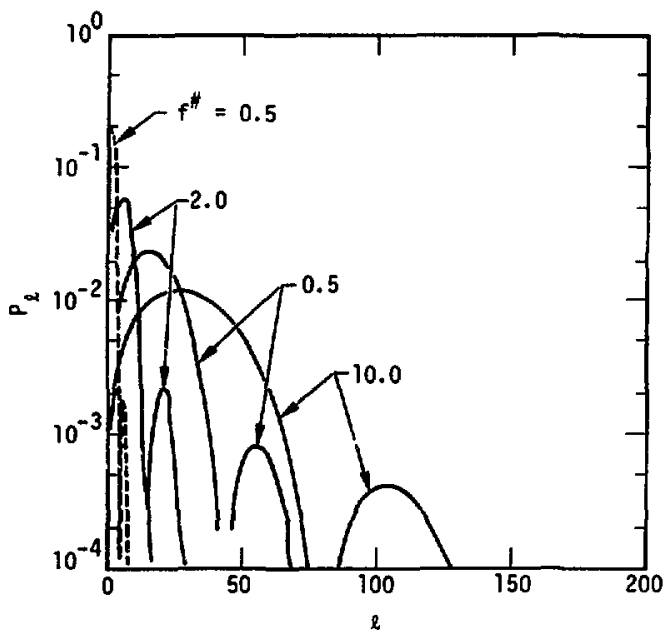

Figure 11.6 Multipole spectra of a focused laser beam. $k \rho=0, f^{*}=0.5$ to 10.0 


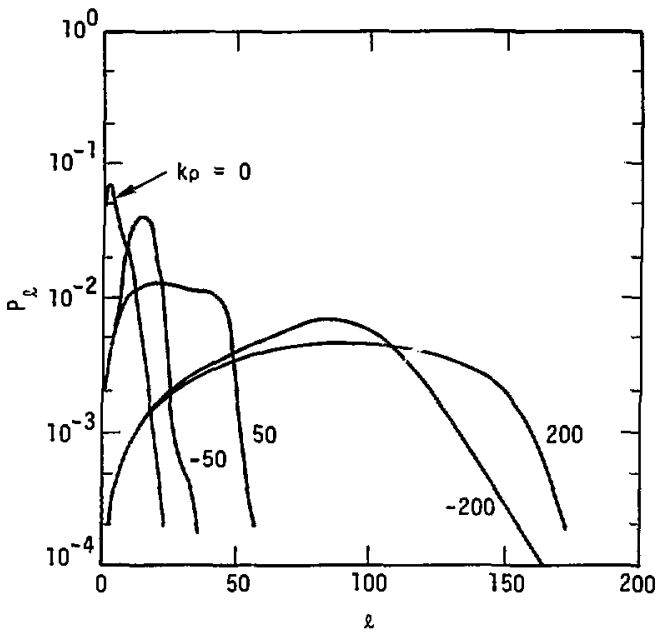

Figure [I.] Multipole spectra of a focused laser beam. $w=1.0, f^{4}=0.5, \mathrm{kp}=-200$ to 200 


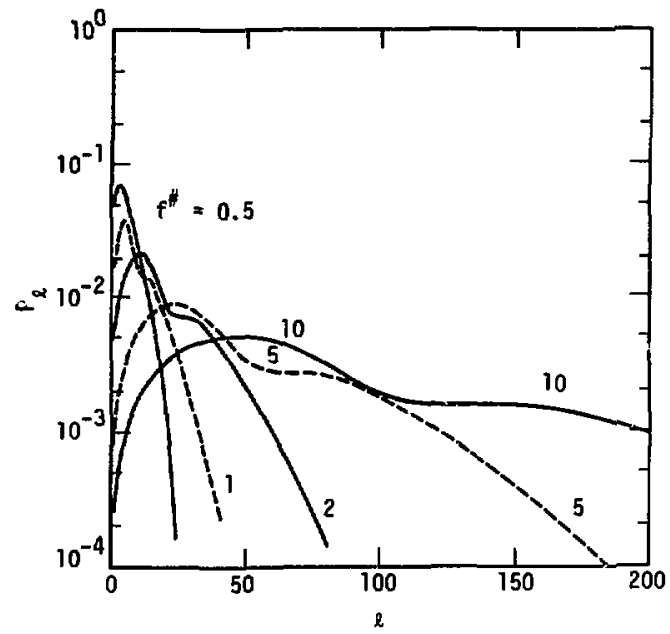

Flgure II.8 Multipole spectra of a focused laser beam. $w=1.0, k \rho=0, t=0.5$ to 10.0 


\section{II. ELECTRONAGNETIC HAVES IN A OENSE, SPHERICALLY SYHAETRIC PLASMA}

\section{A. Introduction}

The propagation of electromagnetic waves in a plasma has been studied for many years in regards to wave propagation in the ionosphere, and the books by BUDDEM (1961) and GINZBURt (1964) are but two among many devoted exclusively to this subject. Here we study electromagnetic waves in an inhomogeneous spherical plasma with a density variation in the radial direction. We will analyze the propagation of laser beams foclised onto this plasma, considerirg energy losses from the electromagnetic wave due to both Joule heating and resonance absorption, which nccurs when the electromagnetic waves directly couple to electron plasma waves at the critical surface of the plasma (where the lioht frequency and the plasma frequency are equal).

This problem was first investigated by DENISOV (1957) and more recently, with various new considerations, by PILIYA (1966), FRIEDBERG, MI TCHELL, MDRSE, and RUDSINSKI (1972), FORSELUNO, KINDEL, LEE, LINORAN, and MORSE (1975) and DEGROOT and TULL (1975). GINZBURF (1964) also covers the subject in considerable depth.

In these references resonance absorption is treated in Cartesian geometry, and the electromagnetic waves and the plasma are planar and infinite in extent. In an attempt to treat this problem in a geometry more closely representative of laser fusion experiments and proposed 
laser fusion systems, we find solutions for spherical electromagnetic waves in a spherically symmetric plasma. Realistic models of laser beams have been constructed from spherical waves in Chapter II, and here we use similar multipole expansions to describe the fields within the plasma, taking the free space solutions as boundary conditions. With this expansion inside the plasma the spherical hamonics continue to describe the transverse variation of the fields, so the three dimensional solutions are found by solving only a one dimensional equation. This is possible, of course, only when the plastra is spherically symmetric.

\section{B. Equations}

The equations we solve are Maxwell's equations in steady state combined with linearized fluid equations for the electrons in the plasma. The full set of equations is:

$$
\begin{aligned}
& \frac{\partial n}{\partial t}+\nabla \cdot \underline{n} \underline{\underline{v}}=0 \\
& m n\left(\frac{\partial \underline{v}}{\partial t}+v \underline{\underline{v}}+\underline{v} \cdot \underline{\nabla v}\right)+\underline{\nabla p}=\mathrm{en} \underline{\underline{E}} \\
& \underline{\nabla} \times \underline{E}=-\frac{1}{c} \frac{\partial B}{\partial t} \\
& \underline{\nabla} \times \underline{B}=\frac{4 \pi}{c} \underline{j}+\frac{1}{c} \frac{\partial E}{\partial \underline{t}} \\
& \underline{\nabla} \cdot \underline{E}=4 \pi p_{c} \\
& \underline{\nabla} \cdot \underline{B}=0
\end{aligned}
$$


$\underline{j}=n e \underline{v}$

$p=\gamma \theta n$

where $n$ is the electron density

$\underline{v}$ is the electron velocity

$m$ is the electron mass

$\gamma$ is the ratio of specific heats

0 is the electron temperature

p is the electron pressure

j. is the current density

$\rho_{c}$ is the charge density

$v \quad$ is a phenomenological damping term, here modeled to represent the inverse bremsstrahlung collision frequency, and we have assumed the adiabatic pressure law for the electrons. Linearizing by means of the substitution

$$
\begin{aligned}
& n=n_{0}+n_{1} e^{-i \omega t} \\
& \underline{v}=\underline{v}_{1} e^{-i \omega t} \\
& \underline{E}=\underline{E}_{0}+\underline{E}_{1} e^{-j \omega t} \\
& \underline{B}=\underline{B}_{0}+\underline{B}_{1} e^{-i \omega t}
\end{aligned}
$$

we derive, for the first order quantities, the coupled equations

$$
\begin{aligned}
& \underline{\nabla} \times \underline{E}=i k \underline{B} \\
& \underline{\nabla} \times \underline{B}=-i k \varepsilon \underline{E}+\frac{a n}{n_{0}} \underline{\nabla} n_{0}-a \underline{\nabla n}
\end{aligned}
$$




$$
\begin{aligned}
& \underline{\nabla} \cdot \underline{B}=0 \\
& \underline{\nabla} \cdot \underline{E}=4 \pi \mathrm{en}
\end{aligned}
$$

where $\quad x=\omega / c$

$$
a=i \frac{4 \pi e}{c} \frac{Y \theta}{m \omega}(1+i v / \omega)
$$

$$
r=1-\frac{\omega_{p}^{2}}{\omega^{2}(1+i v / \omega)}
$$

$$
\omega_{p}{ }^{2}=\frac{4 \pi n_{0} e^{2}}{m}
$$

and we have dropped the subscript 1 .

For a cold plasma, $a=0$, and the equation for $n$ decouples from the equation for the electromagnetic fields. It is inconsistent, however, to use a finite $v$ when $T$ is taken to be zero, as the inverse brejilsstrahiung collision frequency is related to the temperature by the well known expression (DAWSON, KAW, and GREEN (1967)):

$$
v / \omega=24.7 n_{0}(r) /\left(n_{\text {eritical }} T^{3 / 2}\right)
$$

where $T$ is the electron temperature in $2 \mathrm{~V}$ and $\mathrm{n}_{\text {critical }}$ is the electron density at the critical surface. We will require that

$$
|\subset \nabla n| /|k \varepsilon E|
$$

be small in order to attribute validity to our solutions which neglect the second and third terms on the right hand side of equation 3.3.b. FORSELUND et al (1975) have demonstrated that the total absorption by the plasma is negligibly affected by including these terms, and GINZBURG 
(1964) shows how the plasma waves which result when these terms are important act as an effective collision frequency insofar as they limit the amplitude of the electrostatic wave at the critical surface. Later we sha 71 use arguments based on this idea in defining the range of validity of our solutions as a function of temperature.

The final set of equations to be solved is:

$$
\begin{aligned}
& \underline{\nabla} \times \underline{E}=i k \underline{B} \\
& \underline{\nabla} \underline{B}=-i k E \underline{E} \\
& \underline{\nabla} \cdot \underline{B}=0 \\
& \varepsilon=1-\omega_{p}^{2} /\left[\omega^{2}(1+i v / \omega)\right] \\
& \omega_{p}^{2}=\frac{4 \pi n_{0} e^{2}}{m}
\end{aligned}
$$

where

III. C. Solutions in Spherical Coordinates

In sphericat coordinates, these equations have been solved by WYATT (1962) for plane wave scattering from diffuse sphere and ARNUSH (1964), for scattering from plasmas in which the dielectric constant vanishes at some point in the plasma. Although our approach closely parallels that of Wyatt, the applications are closer to those of Arnush and, as neither work treats explicitly our form if the dielectric constant, $\varepsilon$, there is sufficient justification to re-derive the basic equations to be solved.

Consider first solutions for which $E_{r}=0$. Then 


$$
(\underline{\nabla} \times \underline{B})_{r}=0
$$

and we can define a potential $V$ such that

$$
\begin{aligned}
& B_{\theta}=\frac{1}{r} \frac{\partial V}{\partial \theta} \\
& B_{\phi}=\frac{1}{r \sin \theta} \frac{\partial V}{\partial \phi} .
\end{aligned}
$$

Then $\quad-i k B_{\theta}=\frac{1}{r} \frac{\partial}{\partial r} r E_{\phi}$

$$
i k B_{\phi}=\frac{1}{r} \frac{\partial}{\partial r} r E_{\theta} \text {. }
$$

Choosing

$$
V=\frac{1}{i k} \frac{\partial}{\partial r} r \psi
$$

we have

$$
\begin{aligned}
& E_{\phi}=-\frac{\partial \psi}{\partial \theta} \\
& E_{\theta}=-\frac{1}{\sin \theta} \frac{\partial \psi}{\partial \phi} \\
& B_{\theta}=\frac{-1}{i k r} \frac{\partial}{\partial r} \frac{\partial}{\partial \theta} r \psi \\
& B_{\phi}=\frac{-1}{i k r} \frac{1}{\sin \theta} \frac{\partial}{\partial r} \frac{\partial}{\partial \phi} r \psi .
\end{aligned}
$$

From equation 3.5.b

$$
{ }^{i k B_{r}}=\frac{1}{r \sin \theta}\left(\frac{\partial}{\partial \theta} \sin \theta E_{\phi}-\frac{\partial E_{\theta}}{\partial \phi}\right)
$$

or

$$
-i k B_{r}=\frac{1}{r \sin \theta}\left(\frac{\partial}{\partial \theta} \sin \theta \frac{\partial \psi}{\partial \theta}+\frac{1}{\sin \theta} \frac{\partial^{2} \psi}{\partial \phi}\right)
$$


But

$$
\begin{aligned}
& -i k E E_{\phi}=\frac{1}{r} \frac{\partial}{\partial r} r B_{\theta}-\frac{1}{r} \frac{\partial B_{r}}{\partial \theta} \\
& -i k E E_{\theta}=\frac{1}{r \sin \theta} \frac{\partial B_{r}}{\partial \phi}-\frac{1}{r} \frac{\partial}{\partial r} r E_{\phi}
\end{aligned}
$$

SD

$$
\begin{aligned}
& -i k \in E_{\phi}=-\frac{1}{r} \frac{\partial}{\partial r} \frac{1}{i k} \frac{\partial}{\partial r} r E_{\dot{\phi}}-\frac{1}{r} \frac{\partial B_{r}}{\partial \theta} \\
& -i k E E_{\theta}=\frac{1}{r \sin \theta} \frac{\partial B_{r}}{\partial \phi}-\frac{1}{i k r} \frac{\partial}{\partial r} \frac{\partial}{\partial r} r E \theta
\end{aligned}
$$

and

$$
\begin{aligned}
& k^{2} r \in E_{\phi}+\frac{\partial^{2}}{\partial r^{2}} r E_{\phi}=-i k \frac{\partial B_{r}}{\partial \theta} \\
& k^{2} r \varepsilon E_{\theta}+\frac{\partial^{2}}{\partial r^{2}} r E_{\theta}=\frac{i k}{\sin \theta} \frac{\partial B_{r}}{\partial \phi} .
\end{aligned}
$$

Finally, substituting for $E_{\phi}, E_{\theta}$, and $B_{r}$ gives:

$$
\begin{array}{r}
-\frac{\partial}{\partial \theta}\left[k^{2} r \varepsilon \psi+\frac{\partial^{2}}{\partial r^{2}} r \psi\right]=\frac{\partial}{\partial \theta}\left[\frac { 1 } { r \operatorname { s i n } \theta } \left(\frac{\partial}{\partial \theta} \sin \theta \frac{\partial \psi}{\partial \theta}\right.\right. \\
\left.\left.+\frac{1}{\sin \theta} \frac{\partial^{2} \psi}{\partial \phi}\right]\right] \\
\frac{1}{\sin \theta} \frac{\partial}{\partial \phi}\left[k^{2} r \varepsilon \psi+\frac{\partial^{2}}{\partial r^{2}} r \psi\right] \\
=-\frac{1}{\sin \bar{\theta}} \frac{\partial}{\partial \phi}\left[\frac{1}{r \sin \theta}\left(\frac{\partial}{\partial \theta} \sin \theta \frac{\partial \psi}{\partial \theta}+\frac{1}{\sin \theta} \frac{\partial^{2} \psi}{\partial \phi^{2}}\right)\right]
\end{array}
$$


where we have used the fact that $\varepsilon$ is spherically symetric.

Therefore $\psi$ is a solution of:

$$
\frac{\partial^{2} r \psi}{\partial r^{2}}+k^{2} r E \psi+\frac{1}{r \sin \theta}\left(\frac{\partial}{\partial \theta} \sin \theta \frac{\partial \psi}{\partial \theta}+\frac{1}{\sin \theta} \frac{\partial^{2} \psi}{\partial \phi}\right)=0 \quad 3.17 . a
$$

which can be written as

$$
\nabla^{2} \psi+k^{2} \varepsilon \psi=0
$$

Given $\psi$, all field components belonging to the mode $E_{r}=0$ (transverse electric) can be determined.

Next we solve for fields belonging to the transverse magnetic mode $\left(B_{r}=0\right)$ : here $E$ can be written in terms of a potential $U$ such that

$$
\begin{aligned}
& E_{\theta}=\frac{1}{r} \frac{\partial U}{\partial \theta} \\
& E_{\phi}=\frac{1}{r \sin \theta} \frac{\partial U}{\partial \phi}
\end{aligned}
$$

and

$$
\begin{aligned}
i k E E_{\theta} & =\frac{1}{r} \frac{\partial}{\partial r} r B_{\phi} \\
-i k \in E_{\phi} & =\frac{1}{r} \frac{\partial}{\partial r} r B_{\theta} .
\end{aligned}
$$

Choosing

$$
U=\frac{1}{i k \varepsilon} \frac{\partial}{\partial r} r x
$$




$$
\begin{aligned}
& B_{\phi}=\frac{\partial \chi}{\partial \theta} \\
& B_{\theta}=\frac{1}{\sin \theta} \frac{\partial \chi}{\partial \phi} \\
& E_{\theta}=\frac{1}{i k \varepsilon r} \frac{\partial}{\partial r} \frac{\partial}{\partial \theta} r x \\
& E_{\phi}=\frac{1}{i k \varepsilon r} \frac{1}{\sin \theta} \frac{\partial}{\partial r} \frac{\partial}{\partial \phi} r X .
\end{aligned}
$$

From equation 3.5.b

$$
\begin{aligned}
-i k_{E} E_{r} & =\frac{1}{r \sin \theta}\left(\frac{\partial}{\partial \theta} \sin \theta B_{\phi}-\frac{\partial B_{\theta}}{\partial \phi}\right) \\
& =\frac{1}{r \sin \theta}\left(\frac{\partial}{\partial \theta} \sin \theta \frac{\partial x}{\partial \theta}+\frac{1}{\sin \theta} \frac{\partial^{2} x}{\partial \phi}\right) .
\end{aligned}
$$

But

$$
\begin{aligned}
i k B_{\phi} & =\frac{1}{r} \frac{\partial}{\partial r} r E_{\theta}-\frac{1}{r} \frac{\partial E_{r}}{\partial \phi} \\
i k B_{\theta} & =\frac{1}{r \sin \theta} \frac{\partial E_{r}}{\partial \phi}-\frac{1}{r} \frac{\partial}{\partial r} r E_{\phi}
\end{aligned}
$$

so

$$
\begin{aligned}
& i k B_{\phi}=\frac{1}{r} \frac{\partial}{\partial r} \frac{1}{i k \varepsilon} \frac{\partial}{\partial r} r B_{\phi}-\frac{1}{r} \frac{\partial E_{r}}{\partial \theta} \\
& i k B_{\theta}=\frac{1}{r \sin \theta} \frac{\partial E_{r}}{\partial \phi}+\frac{1}{r} \frac{\partial}{\partial r} \frac{i}{i k \varepsilon} \frac{\partial}{\partial r} r B_{\theta}
\end{aligned}
$$

and

$$
-k^{2} B_{\phi}-\frac{1}{r} \frac{\partial}{\partial r} \frac{1}{\varepsilon} \frac{\partial}{\partial r} r B_{\phi}=-\frac{j k}{r} \frac{\partial E_{r}}{\partial \theta}
$$




$$
-k^{2} B_{\theta}-\frac{1}{r} \frac{\partial}{\partial r} \frac{1}{\varepsilon} \frac{\partial}{\partial r} r B_{\theta}=\frac{i k}{r \sin \theta} \frac{\partial E_{r}}{\partial \phi} .
$$

Therefore

$$
\begin{aligned}
-\frac{\partial}{\partial \theta}\left(k^{2} x+\frac{1}{r} \frac{\partial}{\partial r}\right. & \left.\frac{1}{\varepsilon} \frac{\partial}{\partial r} r x\right) \\
& =\frac{\partial}{\partial \theta}\left[\frac{1}{\varepsilon r^{2} \sin \theta}\left(\frac{\partial}{\partial \theta} \sin \theta \frac{\partial x}{\partial \theta}+\frac{1}{\sin \theta} \frac{\partial^{2} x}{\partial \phi}\right)\right] 3.26 . a \\
\frac{1}{\sin \theta} \frac{\partial}{\partial \phi}\left(k^{2} x+\right. & \left.\frac{1}{r} \frac{\partial}{\partial r} \frac{1}{\varepsilon} \frac{\partial}{\partial r} r x\right) \\
& \left.=-\frac{1}{\sin \theta} \frac{\partial}{\partial \phi} \frac{1}{\varepsilon r^{2} \sin \theta} \frac{\partial}{\partial \theta} \sin \theta \frac{\partial x}{\partial \theta}+\frac{1}{\sin \theta} \frac{\partial^{2} x}{\partial \phi}\right) .
\end{aligned}
$$

Again we use the fact that $\varepsilon$ is dependent of $\theta$ and $\phi$, so that

$$
\begin{aligned}
\frac{\partial^{2}}{\partial r^{2}} r X-\frac{1}{\varepsilon} \cdot \frac{\partial \varepsilon}{\partial r} \frac{\partial}{\partial r} r x+k^{2} \varepsilon r x & \\
& +\frac{1}{r^{2} \sin \theta}\left(\frac{\partial}{\partial \theta} \sin \theta \frac{\partial r \chi}{\partial \theta}+\frac{1}{\sin \theta} \frac{\partial^{2} r x}{\partial \phi^{2}}\right)=0
\end{aligned}
$$

which we can also write in the form

$$
\nabla^{2} X-\frac{l}{\varepsilon r} \frac{\partial E}{\partial r} \frac{\partial}{\partial r} r X+k^{2} E X=0
$$

These results are summarized in Table 3.1.

The equations for the potentials are solved as follows: the angular derivatives in equations 3.17 and 3.27 have as eigenfunctions the spherical harmonics. As a result, we can separate variables by means of the substitutions: 


$$
\begin{aligned}
x(r, \theta, \phi)= & \sum_{\ell=0}^{\infty} x_{\ell}(r)\left[a_{\ell} p_{\ell}(\cos \theta)\right. \\
& \left.+\sum_{m=1}^{\ell}\left(a_{\ell}^{m} \cos m \phi+b_{\ell}^{m} \sin m \phi\right) p_{\ell}^{m}(\cos \theta)\right] \quad 3.29 . a \\
\psi(r, \theta, \phi)= & \sum_{l=0}^{\infty} y_{\ell}(r)\left[c_{\ell} p_{\ell}(\cos \theta)\right. \\
& \left.+\sum_{m=1}^{\ell}\left(c_{\ell}^{m} \cos m \phi+d_{\ell}^{m} \sin m \phi\right) P_{\ell}^{m}(\cos \theta)\right]
\end{aligned}
$$

which requires that $x_{\ell}$ and $y$. satisfy

$$
\begin{aligned}
& \frac{d^{2}}{d r^{2}} r x_{\ell}-\frac{1}{r} \frac{d \varepsilon}{d r} \frac{d}{d r} r x_{\ell}+k^{2} c r x_{\ell}-\frac{l(\ell+1)}{r^{2}} r x_{\ell}=0 \\
& \frac{d^{2}}{d r^{2}} r y_{\ell}+\left(k^{2} \varepsilon-\frac{\ell(\ell+1)}{r^{2}}\right) r y_{\ell}=0 .
\end{aligned}
$$

In Chapter II we found that an axisymetric laser beam had only $m=1$ components in its Debye potential expansion, so that for a linearly polarized incident beam, $x$ and $\psi$ can be expanded in terms of the $\cos \phi$ and $\sin \phi$ functions only. Using this expansion, we find the results surmarized in Table 3.2 .

There is a close relationship between the Debye potential function differential equations in spherical coordinates and the corresponding field equations in Cartesian coordinates. Equation 3.30.a for the TM potential function $x_{2}$ is identical to equation (1) of DENISOV (1957). 
which describes the magnetic field normal to the plane of incidence for a plane wave obliquely incident at an angle $\theta_{0}$ from normal onto an inhomogenious slab plasma, if we replace $r x_{\ell}$ by Btransverse and $\ell(\ell+1) / r^{2}$ by $k^{2} \sin ^{2} \theta_{0}$.

Similarly, equation 3.30.b corresponds to the electric field equation for a plane wave polarized with its electric field normal to the plane of incidence, as given by GINZBURG (1964), equation 16.8 , if $r_{\ell_{2}}$ is replaced by Etransverse.

We interpret the spherical Debye potential equations as follows: first, the electric fields resulting from the TE potential $(\psi)$ have no components in the radial direction, and therefore no plasma waves can be generated by this wave in a spherically symmetric plasma. The solution to the radial part of the TE potential equation has a cutoff at the radius given by

$$
k^{2} \varepsilon(r)=\ell(\ell+1) / r^{2}
$$

For $\varepsilon$ increasing with $r$ in the plasma, higher multipole fields are cutoff and reflected at successively larger radii, and the fields generated by these potentials, for equivalent source strength, are successively weaker in the vicinity of the critical surface. The incident and reflected waves then interfere to form standing waves, and when the thickness of the atmosphere is small relative to the plasma radius (so that $\ell(\ell+1) / r^{2}$ changes little across the atmosphere) these waves are identical to the standing waves found in Cartesian coordinates (which for $\varepsilon$ varying linearly with $r$ gives the well known Airy function solution (BUDDEN (1961) p. 283). 
On the other hand, the TM potential functions generate the radial electric field which resonantly couples to plasma waves at the critical surface, leading to energy loss from the electromagnetic wave. This wave also has a cutoff outside the critica? surface, but evanescont waves tunnel through to the critical surface where their radial electric fields generate the electron plasma waves.

Although the higher multipole fields are again reflected at successively greater and greater radii, the radial electric field carried by the $\ell$-th multipole is proportional to $\ell(\ell+1)$. For small $\ell$ this factor more than compensates for the increased attenuation resulting from the cutoff, and the radial field which exists at the critical surface initially increases with $\ell$. Only for $\ell$ of order $\mathrm{kr}_{\mathrm{c}}$ does the cutoff significantly affect the wave at the critical surface $\left(r_{c}=\right.$ radius of critical surface). Using the correspondence with Cartesian coordinates, we take the formula of DENISOV(1958)-FRIEDBURG (1972) to predict the multipole for which resonance absorption is maximized. When the density gradient is 1 inear and the target radius is large compared to the atmospheric scale length, we have

$$
\ell_{\max }\left(l_{\max }+1\right)=0.5\left(\frac{r_{C}}{\Delta R}\right)^{2}(k \Delta R)^{4 / 3}
$$

where $\Delta R$ is the atmospheric thickness. For example, with $r_{c}=100 \mu m$ and $\Delta R=50 \mu m$, the maximum resonance absorption occurs at $\ell=65$; changing $\Delta R$ to $5 \mu m$ gives maximum resonance absorption at $\ell=140$.

He will delay the discussion of specific examples, which will follow a brief description of the numerical scheme employed to solve equations 3.30 . 
III. D. Numerical Solutions

To determine the electromagnetic fields in all space, we must solve equations 3.30.a and 3.30.b subject to the boundary conditions of Section II. The fields, then, follow from the formulae of Tables 3.1 and 3.2. Consider first equation 3.30.b which, for

$$
\begin{aligned}
& w=r y_{\ell}, \\
& u=d w / d r,
\end{aligned}
$$

is written as

$$
\frac{d u}{d r}+\left(k^{2} E-\frac{\ell(\ell+1)}{r^{2}}\right) w=0
$$

At the boundary of the plasma, $r=R$, the wave consists of an incident (specified) wave and a scattered wave, which is purely outgoing. Because the free space solutions to equation 3.30.b are spherical Bessel functions, the outing wave must be a spherical Hankel function of the first kind, $h_{l}^{(1)}$, and from the Bessel function recursion relations, it follows that, at $r=R$

$$
\begin{aligned}
\frac{d w}{d r}+\left(\frac{\ell}{R}-\right. & \left.k \frac{h_{\ell-1}^{(1)}(k R)}{h_{\ell}^{(1)}(k R)}\right) w \\
& =\frac{d w}{d r}^{i n c}+\left(\frac{\ell}{R}-k \frac{h_{\ell-1}^{(1)}(k R)}{h_{\ell}^{(1)}(k R)}\right) w^{i n c},
\end{aligned}
$$

where the right hand side is a known quantity. 
The second boundary condition, based on the finiteness of the solution at the origin, is

$$
w(r=0)=0 .
$$

Therefore, defining

$$
\begin{aligned}
& v=u+b w \\
& b=\frac{\ell}{R}-k \frac{h_{\ell-1}^{(1)}(K R)}{n_{l}^{(1)}(K R)}
\end{aligned}
$$

and

$$
c=k^{2} \varepsilon-\ell(\ell+1) / r^{2}
$$

we can write

$$
\begin{aligned}
& \frac{d u}{d r}=\frac{c}{b} u-\frac{c}{b} v \\
& \frac{d v}{d r}=\left(b+\frac{c}{b}\right) u-\frac{c}{b} v
\end{aligned}
$$

subject to boundary conditions

$$
\begin{aligned}
& v(0)=u(0) \\
& v(R)=\frac{d w}{d r}^{\text {inc }}+b w^{\text {lnc }} .
\end{aligned}
$$

We solve equations 3.38.a and 3.38.b numerically starting at $r=0$ with $u=v=I$ (arbitrary), and integrate from $r=0$ to $r=R$, at which point the amplitude and phase of $v$ is known by the second boundary 
condition. We then sweep back through the array of $u$ and $v$ values, rescaling according to the known boundary conditions.

The quantity needed for the series expansion of the fields is

$$
y_{\ell}=\frac{v-u}{\text { br }} .
$$

To solve equation 3.29.a, define:

$$
\begin{aligned}
& w=r x_{\ell} \\
& u=\frac{1}{E} \frac{d w}{d r}
\end{aligned}
$$

and

$$
v=\varepsilon u+b w,
$$

so then

$$
\begin{aligned}
& \frac{d u}{d r}=\frac{c}{b} u-\frac{c}{c b} v \\
& \frac{d v}{d r}=\left(b+\frac{c}{b}+\frac{1}{r} \frac{\partial f}{\partial r}\right) \varepsilon u-\frac{c}{b} v
\end{aligned}
$$

subject to

$$
\begin{aligned}
& v(0)=\varepsilon(0) u(0) \\
& v(R)=\frac{d w}{d r}^{\text {inc }}+b w^{\text {inc }}
\end{aligned}
$$

and the solution proceeds as above.

Use of the variables chosen insures the continuity of the transverse components of $E$ and $B$ in all space. However, as v/w approaches 
zero, the shell in which the resonantly driven fields are large becomes very thin, and it is necessary to refine the radial grid coordinate. Several solutions are considered in the appendix as a means of verifying the technique:

(1) For E approaching unity in all space, the solutions to both equations 3.30.a and 3.30.b are the spherical Bessel functions, (JACKSON (1962),p.539), matching the amplitude and the phase of the incident wave at $r=R$. In Appendix 3 we compare the numerical solutions of equation 3.30 with the spherical Besse? functions.

(2) For a linear gradient, it has been shown by BUDOEN (7961) p.283, as well as may others, that the solutions to equation 3.30.b are Airy functions under the conditions described earlier. He compare our solutions with the Airy function solutions in Appendix 4.

(3) FRIEDBURG et al (1972) numerically solved the Cartesian equivalent of equation 3.30.a. Again we duplicate the proviously known solution as a means of verifying the techniques used here. (Appendix 5)

III. E. Results

He have found that the potential functions for the electromagnetic fields in a plasma can be described as the series sum of the product of an unknown radial function and a spherical harmonic function, the radial function being the solution to equation 3.30. Eventually, in order to 
reconstruct the fields scattered by the plasma, we will combine the incident wave multipole spectra, which are given in Chapter II, with the fields given by these potential functions. First, however, we will consider in more detail the properties of the radial functions $x_{l}$ and $y_{\ell}$, as a function of the plasma parameters $r_{c}, \Delta R$ and $v / \omega$.

Throughout this report we will maintain as many parameters as possible at or near the same numerical values. Our "standard" plasma will have $r_{c}=100 \mu \mathrm{m}, \Delta R=50 \mu \mathrm{m}$ and $T=850 \mathrm{eV}(\nu / \omega=.001)$. For the $x_{\ell}$ and $y_{\ell}$ plots we mainly use $\Delta R=5 \mu m$ however, since fewer standing waves exist in the plasma and the plots are cunsenuently easier to read. We take the laser wavelength to be $1 \mu \mathrm{m}$ and the density gradient, linear. In Figure III. 1 we plot $\left|x_{\ell}\right|^{2}$ and $\left|y_{\ell}\right|^{2}$ for $\ell=7,14 C, 300$, and 600 and $r_{c}=100 \mu \mathrm{m}, \Delta R=5 \mu \mathrm{m}$, and $v / \omega=.001(T=850 \mathrm{eV})$. Even though stroniz resonance absorption is occurring at $\varphi=140$, little change is seen in the plots of $\left|x_{\ell}\right|^{2}$ and $\left|y_{\ell}\right|^{2}$. It is the term $1 / E$ in the equatiuns for $E$ in Tables 3.1 and 3.2 which amplifies the effect of resonance absorption. The effect of the plasma cutoff is easily observed in the differences between $\ell=140,300$ and 600 plots.

By changing $\Delta R$, the number of standing waves in the plasma changes, and as $\Delta R$ becomes larger, the height and breadth of the wave nearest the crit.cal surface increases, significantly increasing the energy dissipation by classical collisions there. This swelling of the standing waves near the critical surface is a very important factor in the overall efficiency of absorption of a plasma. 
In Figure III.2 we plot, on a different scale, the standing waves for $r_{c}=100, \Delta R=50$, and $v / \omega=0.001$ and $l=1$. (Note that the radial scale is lengthened $\times 10$ from the previous plots). The amplitude and width of the first standing wave away from the critical surface have each more than doubled from the previous plot. The uneven appearance of the envelope plot of this wave is a result of the coarse grid spacinq used in this calculation, and with better resolution, would become smooth.

To observe the effect of temperature on the standing waves, we plot for $\ell=1$ and $r_{c}=100 \mu \mathrm{m}, \Delta R=5 \mu \mathrm{m}$ the waves for $v / \omega=.0001, .01$, .05 and 0.1 ( $T=4000,380,60$ and $40 \mathrm{ev})$ in Figure III. 3 .

At high temperatures the collision frequency is small and little absorption occurs (since there is negligible resonance absorption for $\ell=1)$. However for successively lower plasma temperatures, the effect of the increasing inverse bremsstrahlung collision frequency can be seen in the damping of these standing waves.

In Section II. B we derived an expression for the power in an incident light beam in terms of the coefficients of its Debye potential functions. From the values of $x_{\ell}$ and $y_{\ell}$ on the boundary of the plasma we can define similar expansion coefficients for the outgoing (scattered) waves and, since the rate of energy dissipation in the plasma must be equal to the difference between the incoming and outgoing power, we can use these coefficients to give us the $\ell$ dependence of the power absorption. He could not, for instance, speak similz:ily of the $\ell$ dependence of the field strength, because of interference effects 
between the different multipoles. For the power, however, we showed that the effect of the integration over the transverse coordinates enables us to identify the power associated with each multipole in terms of the expansion coefficients of $X$ and $\psi$. When we do this for the $x_{\ell}$ and $y_{\ell}$ terms separately, we see that the $\psi$ mode loses energy only to collisions, while for some $\ell$ 's the $x$ mode can loose up to $50 \%$ of its power (for linear gradients) through coupling to plasma waves (in our cold plasma madel the plasma waves are themselves collisionly damped).

As mentioned earlier, the optimum $\&$ for coupling is determined by a tradeoff between the strength of the radial electric field driving the plasma waves, which is increasing with $\ell$, and the increasing cutoff radius, which as $\ell$ increases causes higher attenuation of the field between the cutoff and the critical surface.

In Figures 111.4 and III.7 we plot the rate of energy dissipation as a function of $\&$ for the $x$ and $\psi$ modes, for several values of $v / \omega$ and for $\Delta R=5 \mu m$ and $\Delta R=50 \mu m$.

From this set of figures, several effects should be noted: first, the resonance absorption can clearly be identified in the $x$ plots and the value of $\ell$ at which it is greatest is seen to vary with $\ell$ exactly as given in equation 3.32. Secondly, the dependence of classical absorption on $\Delta R$ (because of swelling) also is clearly noted here, as the large $\Delta R$ plasma absorbs energy classically far more efficiently than the plasma with a smaller AR. Finally, at high \&'s we see the effect of the plasma cutoff which prevents the wave from reaching the critical surface, and causes the falloff in both resonance and classical absorption. 
III. F. Sumnary

We have found solutions to Maxwell's equations for an arbitrary spherical wave incident onto a spherically symmetric (radially inhomogenious), overdense plasma. We calculate both resonant absorption and classical absorption via inverse bremsstrahlung. The plasma is described by tinearized fluid equations for the electrons and ions are assumed fixed. The equations we solve are actually the cold plasma equations, but temperature enters parametrically through the size of the inverse bremmstrahlung collision frequency. The fields are defined in terms of the Debye potential functions, whose angular variation is given by the spherical harmonics and whose radial dependence is determined by numerical solution of ordinary differential equations. He discuss the radial dependence of the Debye functions which are analagous to the TE and TM solutions in Cartesian geometry, and present results showing the dependence on various plasma parameters. 
TABLE 3.1

Solutions to Maxwel]'s Equations in Spherically Symmetric Media

T.M. Contribution

$E_{r}=-\frac{1}{i k E r} \frac{1}{\sin \theta}\left(\frac{\partial}{\partial \theta} \sin \theta \frac{\partial x}{\partial \theta}+\frac{1}{\sin \theta} \frac{\partial^{2} x}{\partial \phi}\right)+$

$E_{\theta}=\frac{1}{i k E r} \frac{\partial}{\partial r} \frac{\partial}{\partial \theta} r x$

$E_{\phi}=\frac{1}{i k E r} \frac{1}{\sin \theta} \frac{\partial}{\partial r} \frac{\partial}{\partial \phi} r_{X}$

$B_{r}=$

0

$B_{\theta}=-\frac{1}{\sin \theta} \frac{\partial x}{\partial \phi}$

$B_{\phi}=\frac{\partial x}{\partial \theta}$
T.E. Contribution

0

$\frac{1}{\sin \theta} \frac{\partial \psi}{\partial \phi}$

$-\frac{\partial \psi}{\partial \theta}$

$+\quad-\frac{1}{i k r} \frac{1}{\sin \theta}\left(\frac{\partial}{\partial \theta} \sin \theta \frac{\partial \psi}{\partial \theta}+\frac{1}{\sin \theta} \frac{\partial^{2} \psi}{\partial \phi^{2}}\right)$

$\frac{1}{i k r} \frac{\partial}{\partial r} \frac{\partial}{\partial \theta} r \psi$

$\frac{1}{\mathrm{ikr}} \frac{1}{\sin \theta} \frac{\partial}{\partial r} \frac{\partial}{\partial \phi} r \psi$

where

$$
\begin{aligned}
& \frac{\partial^{2} n \psi}{\partial r^{2}}+\frac{1}{r^{2} \sin \theta}\left(\frac{\partial}{\partial \theta} \sin \theta \frac{\partial \psi}{\partial \theta}+\frac{1}{\sin \theta} \frac{\partial^{2} \psi}{\partial \phi^{2}}\right)+k^{2} \varepsilon r \psi=0 \\
& \frac{\partial^{2} r \chi}{\partial r^{2}}+\frac{1}{r^{2} \sin \theta}\left(\frac{\partial}{\partial \theta} \sin \theta \frac{\partial \chi}{\partial \theta}+\frac{1}{\sin \theta} \frac{\partial^{2} \chi}{\partial \phi^{2}}\right)-\frac{1}{\varepsilon} \frac{\partial \varepsilon}{\partial r} \frac{\partial}{\partial r} r \chi+k^{2} \varepsilon r \chi=0
\end{aligned}
$$


TABLE 3.2

Solutions to Maxwell's Equations in Spherically Symmetric Media for Linearly Polarized Axisymetric Light

T.M. Contribution

$$
\begin{aligned}
& E_{r}=\frac{\ell(\ell+1)}{i k \varepsilon r} x_{\ell} p^{1} \cos \phi \\
& E_{\theta}=\frac{1}{i k E r} \frac{\partial}{\partial r} r x_{\ell}\left[\eta(\ell+1) p_{\ell}^{0}-p_{\ell}^{2}\right] \frac{\cos \phi}{2}+ \\
& E_{\phi}=-\frac{1}{i k \in r} \frac{1}{\sin \theta} \frac{\partial}{\partial r} r x_{\ell} P_{\ell}^{1} \sin \phi \\
& B_{r}=\quad 0 \\
& B_{\theta}=\frac{1}{\sin \theta} x_{\ell} p_{\ell}^{l} \sin \phi \\
& B_{i}=x_{\ell}\left[\ell(\ell+1) P_{l}^{0}-P_{\ell}^{2}\right] \frac{\cos \phi}{2}
\end{aligned}
$$

where $P_{\ell}^{m}$ is the Legendre polynomial

and

$$
\begin{aligned}
& \frac{d^{2}}{d r^{2}} r y_{\ell}+\left[k^{2} \varepsilon-\frac{\ell(\ell+1)}{r^{2}}\right] r y_{\ell}=0 \\
& \frac{d^{2}}{d r^{2}} r x_{\ell}-\frac{1}{\varepsilon} \frac{d \varepsilon}{d r} \frac{d}{d r} r x_{\ell}+\left[k^{2} \varepsilon-\frac{\ell(\ell+1)}{r^{2}}\right] r y_{\ell}=0
\end{aligned}
$$

T.E. Contribution

E 

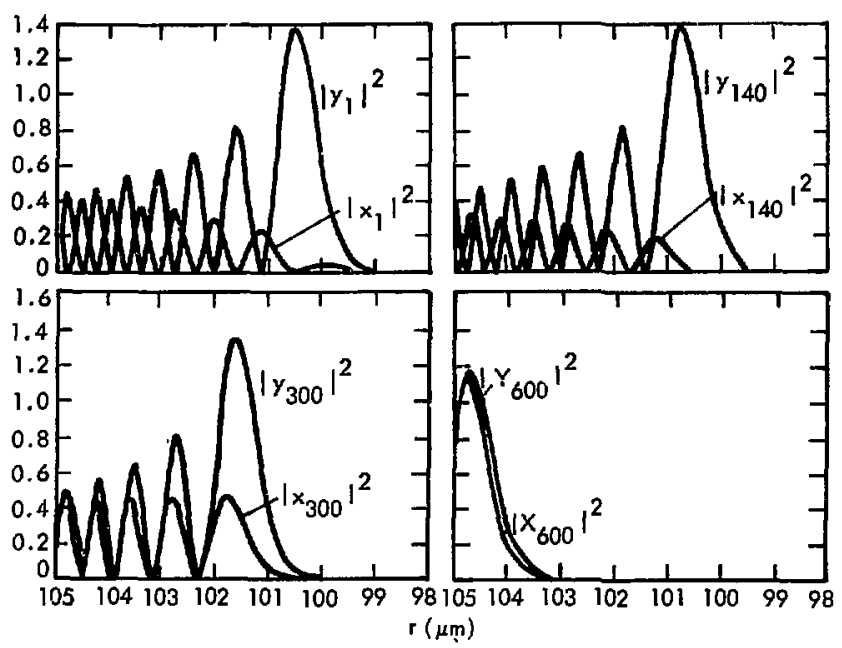

$$
\begin{aligned}
\left|x_{\ell}\right|^{2} \text { and }\left|y_{\ell}\right|^{2} \text { versus } \mathrm{s} & \\
r_{\mathrm{C}}= & 100 \mu \mathrm{m} \\
\Delta \mathrm{R}= & \\
v / \omega= & 0.00 ? \\
T= & 850 \mathrm{eV} \\
1= & 1,140 \\
& 300,600
\end{aligned}
$$

Figure III.? Variation of $\left|x_{\ell}\right|^{2}$ and $\left|y_{\ell}\right|^{2}$ with $\ell, \Delta R=5$ um. 


$$
r_{c}=100 \mu \mathrm{m}, \Delta R=50 \mu \mathrm{m}, T=850 \mathrm{eV}
$$
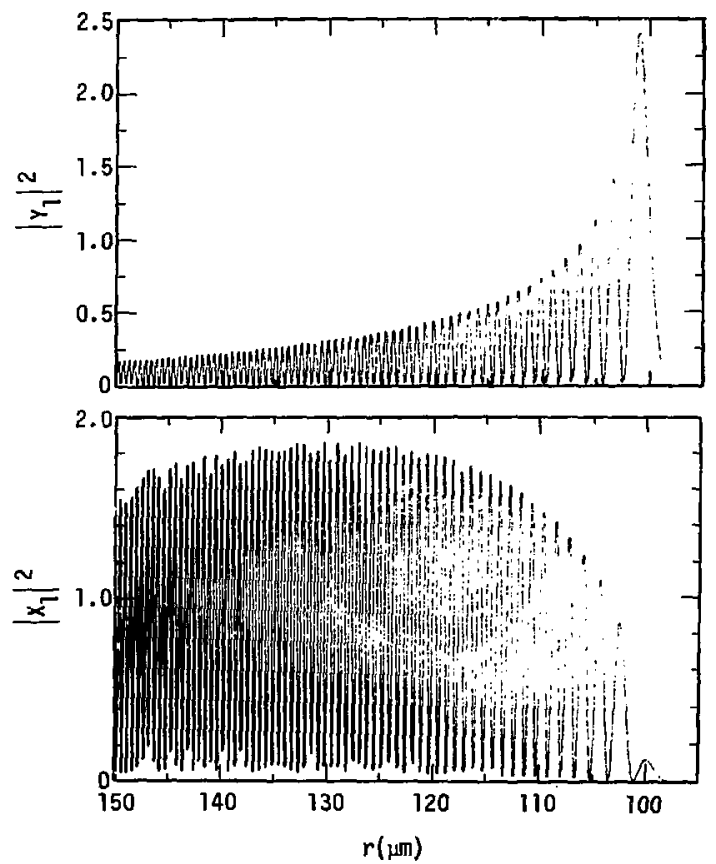

Figure III.2 $\underset{50 \text { variation of }}{\text { Va }}\left|x_{1}\right|^{2}$ and $\left|y_{1}\right|^{2}$ with $r, \Delta R=$ 


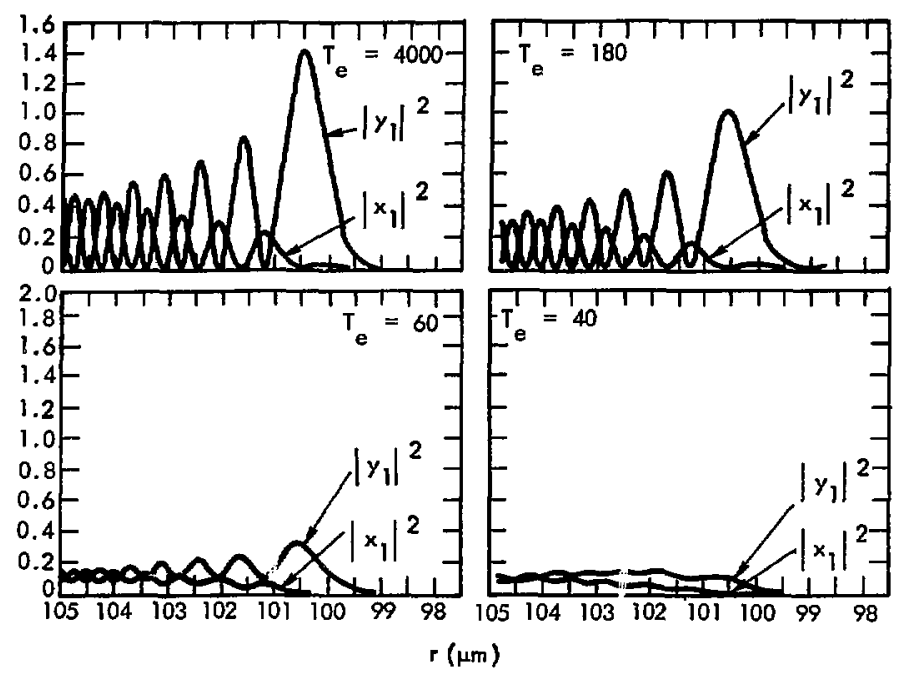

$$
\begin{aligned}
\left|x_{\ell}\right|^{2} & \text { and }\left|y_{\ell}\right|^{2} \text { versus } r \\
r_{C} & =100 \mu \mathrm{m} \\
\Delta R= & 5 \mu \mathrm{m} \\
l= & \\
T= & 4000,180,60 \\
& \text { and } 40 \mathrm{ev}
\end{aligned}
$$

Figure III.3 Effect of temperature on $\left|x_{1}\right|^{2}$ and $\left|y_{1}\right|^{2}$. 


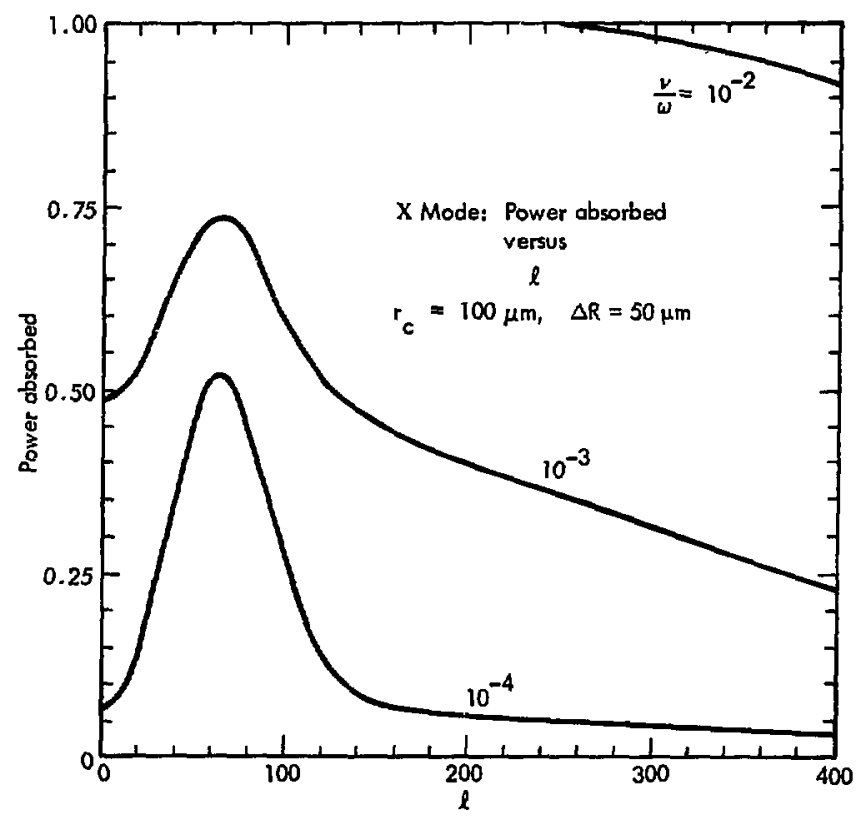

Figure I1I.4 $x$ mode: Power absorbed versus $\ell$. $r_{c}=100 \mathrm{\mu m}, \Delta R=50 \mathrm{\mu m}$. 


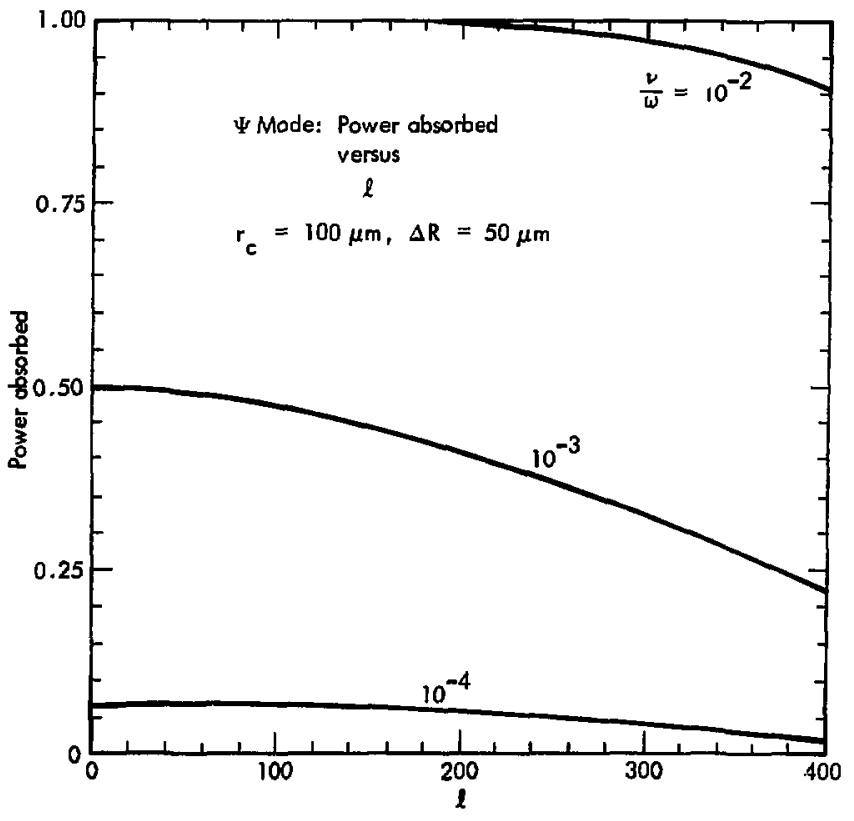

Figure III.5 $\Psi$ mode: Power absorbed versus $\ell$. $r_{c}=100 \mu \mathrm{m}, \Lambda R=50 \mu \mathrm{m}$. 


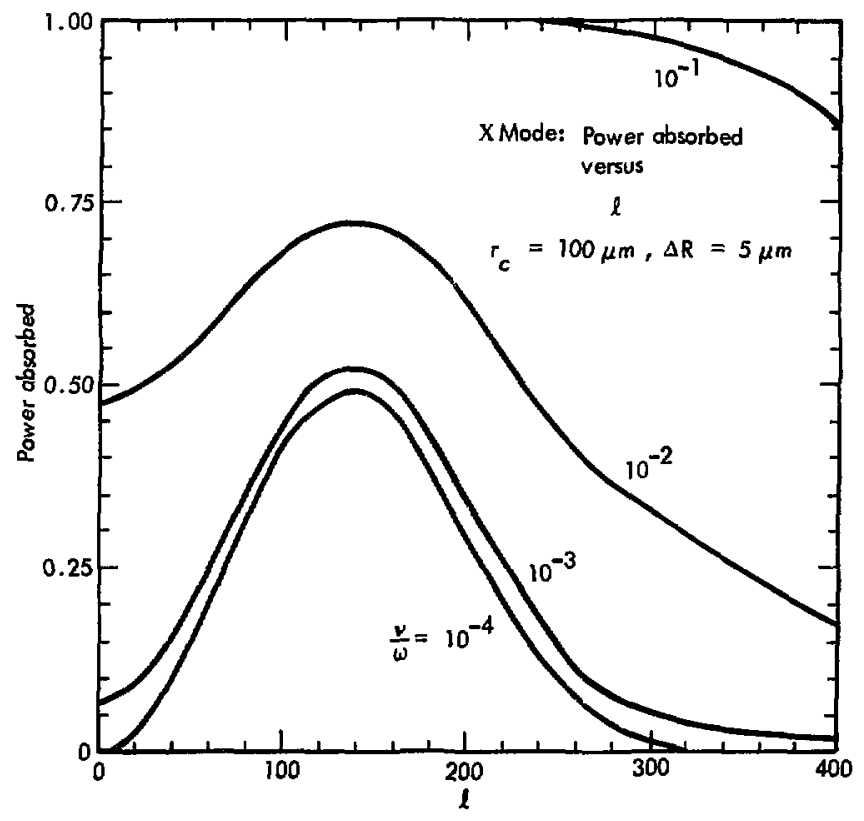

Figure III.6 $x$ mode: Power absorbed versus $\&$ $\hat{r}_{c}=100 \mu \mathrm{m}, \Delta \mathrm{R}=5 \mu \mathrm{m}$. 


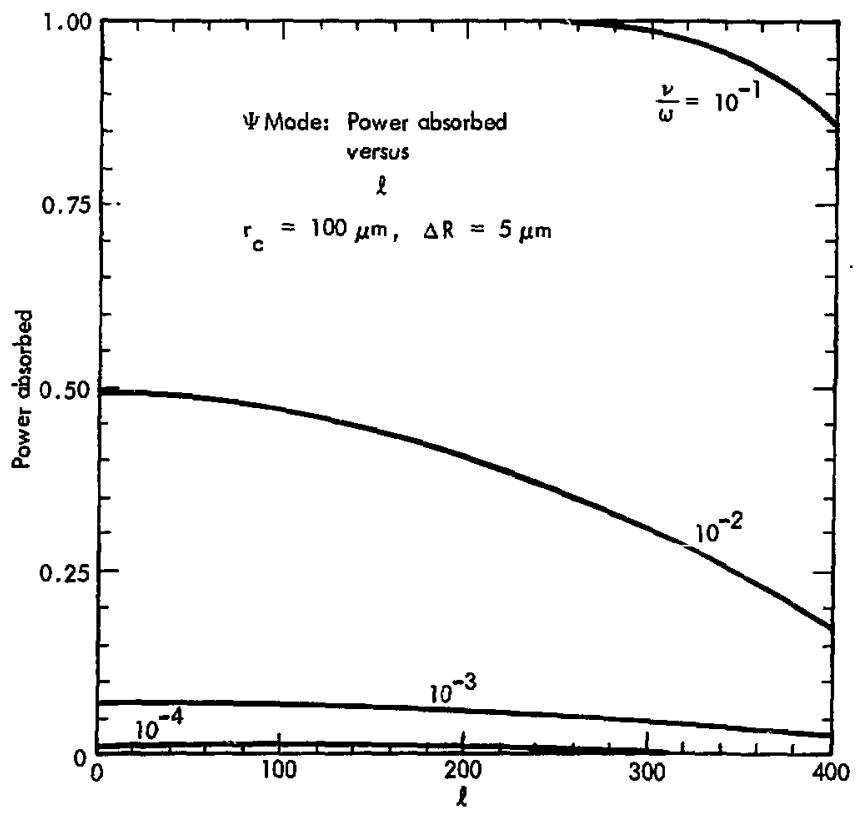

Figure III.7 $\psi$ mode: Power absorbed versus $\&$ $r_{c}=100 \mu \mathrm{m}, \Delta R=5 \mu \mathrm{m}$. 


\section{LASER LIGHT SCATTERING AND ABSORPTION}

\section{A. Introduction}

Thus far we have studied separately new usethods for describing a laser beam in terms of its multipole moments about an arbitrary origin, and for determining the electromagnetic fields in a spherically symmetric plasma by means of a partial wave expansion of the Debye potential functions. In this chapter, these methods sre combined to examine laser light scattering and absorption by spherically symmetric plasmas, which will follow in three sections. First we will analyze the distribution of light scattered by a spherically symmetric plasma which is being illuminated by a single focused laser team, and determine the amount of iight absorbed, and the amount emerging in the forward and the backward directions, disregarding for the moment the spatial distribution of the fields in the plasma. We will consider the effects of (1) plasma translations relative to the beam focus, (2) the $f \#$ of the focusing lens, (3) plasma atmospheric scale length, (4) plasma temperature and (5) spherical aberrations on the incident beam.

Next we will consider the spatial distribution of the fields themselves within the plasma, and following directly from this, the distribution of the energy deposition by the beam, as a function of these parameters.

Finally we will estimate the limitations of the linearized, steady state solutions by calculating the incident beam power for which the 
linearization and steady state assumptions break down. The equations solved here are very good approximations to the full nonlinear set of equations when the beam power is low, and we will determine the approximate power levels at which the various nonlinear terms become important.

IV. B. Total Absorption and Scattered Light Distribution

In Figure IV. I we again illustrate the geometrical definition of $\rho$, the plasma translation. For positive $\rho$ the laser is focused behind the center of the plasma. The fraction of the incident power which is absorbed, scattered (or propagates directly by the plasma) into the forward hemisphere, and scattered into the backward hemisphere (to the left) is plotted in Figure IV. 2 for a plasma with $\Delta R=50 \mu m, r_{c}=100 \mu \mathrm{m}$, $T=850 \mathrm{eV}$ and $\mathrm{f} \#=0.5$. This plot and similar plots which follow consist of two curves. The separation between the $x$-axis and the lower curve is the fraction of the incident light which is absorbed, for that value of $\rho$. The second curve is a plot of the fraction of the incident light beam which emerges in the forward direction, either by diffracting around the plasma or by scattering in the forward direction, and is measured from the top of the graph. The remaining light, which is the fraction of the incident beam which back scatters, is given by the distance between these two curves.

For $\rho$ near zero all the light is either absorbed or backscattered, and small translations to the left or to the right increase the total absorption, because of resonance absorption. Once the incident light multipole distribution is optimal for resonance absorption, further 
translations of the plasma then lead to a diminishing absorption, as the incident power begins to appear in higher multipoles. Some of the light which had been back scattered at the critical surface now begins to scatter into the forward direction and ultimately large fractions of the incident beam miss the plasma altogether.

Looking more closely at the plasma translations to the right ( $\rho$ negative), we see that the absorption is symmetric with respect to $\rho=0$ (for non-aberrated beams), but that the surface of the plasma which is $i 11$ uminated by the laser is vastly different, with the laser focused down to a very small area for $\rho=-100 \mu m$. This causes the fraction of light forward and backscattered to be quite asymetrical with respect to $\rho=0$. Large negative translations also eventually cause forward scattered iight, and light directly propagating into the forward hemisphere.

Before analyzing the spatial resolution of the scattered light, we will consider the effect of plasma temperature on the absorption and scattering, in Figure IV. 3. At lower temperatures the absorption is enhanced for all plasma positions, and resonance absorption is itself decreased in absolute magnitude, because of attenuation of the incident beam prior to reaching thc critical surface. In Figure IV. 4 we plot the same sequence for $\Delta R=5 \mu \mathrm{m}$. The general characteristics of the plot are the same but the overall absorption is considerably less as a result of the diminished wave swelling between $\Delta R=50 \mu \mathrm{m}$ and $\Delta R=5 \mathrm{pm}$. Increasing the $f \#$ of the focusing system has the predictable effect of changing the translational scale, since the illuminated area on the plasma surface changes more gradually with changes in p. Adding 
spherical aberrations to the beam also leads to understandable changes in these plots. For badly aberrated beams, even $p=0$ multipole spectra of the incident beam will have power which is efficiently resonantly absorbed, raising the overall absorption for small values of p. For large spheres (compared to a wavelength) and large translations, the overall effect of aberrations on the absorption is minor. These two effects are illustrated in Figure IV.5.

We plot the forward and backscattered light as functions of the angles $\alpha$ and $\beta, \beta$ being the vertical deflection from the axis of symetry, ranging from $-\pi / 2$ to $t \pi / 2$ and, a likewise, horizontally from the axis of symmetry. All contour plots are symmetrical about the $\alpha=0$ and $\beta=0$ axes, and one can envision the plots as representing the contours on the hemisphere to an observer looking forward (or backward) from the plasma center. Contour values are separated by $10 \%$ of their peak value, and the linear plots to the right and above the contour maps show the intensity on the axes $\alpha=0$ and $\beta=0$, respectively. In the plots of scattered light given in Figures IV.6 through IV.14 we can follow the effects of translation.

In Figure IV.6 through IV.II we plot the backscattered light for situations in which most of the incident light has been intercepted by the plasma and there is no forward scattered light. In Figure IV.6 the light is focused to a small spot on the surface of the plasma, which is essentially planar, and the reflected light scatters backward into a smanl angle. As the focus is moved to the right, we can observe how the backscattered light reflects into larger and larger angles, until in Figure IV. 11 some of the reflected light is forward scattered (very near 
$\pi / 2)$. The effect of the resonance absorption can be seen as follows. For $\rho$ 's for which resonance absorption occurs, the vertically polarized incident light in the horizontal plane is reflected but in the vertical plane is partially absorbed (since the electric field has components along a density gradient only in the verical direction). Therefore, reflected light contours in the vertical direction are depressed, relative to the horizontal. He can see clearly the effect of resonance absorption in Figures IV.6, and IV.9 through IV.12. In Figure IV.7 the phase fronts of the incident light and the plasma surface coincide and the resonance absorption is minimum. Hence the reflected light distribution is symmetric with respect to the axis of the laser.

As we increase $p$ the asymmetry resulting from resonance absorption is easily observed in the backscattered light.

Looking at forward scattered light, we first see light in angles near $\alpha= \pm \pi / 2$ and $\beta= \pm \pi / 2$. This derives from light which has been reflected at the critical surface. As soon as the translations are large enough that unreflected light propagates directly into the forward hemisphere, the diffracted fields caused by this light begin to dominate the forward scattered field distribution. This problen, of the diffracted fields due to a plane wave incident on an annular cpening, is discussed in Appendix 1, and the resulting far field distribution is similar to the Airy pattern caused by a circular hole. As $p$ increases, the thickness of the annular ring increases and the total power diffracted into the Airy pattern increases accordingly. Forward scattered light is plotted in Figures IV.13 and IV.14 on a crude scale, with the details of the forward scattered central spot plotted in Figure IV.15. 
Another important aspect of light scattering in spherical plasmas concerns the effects of refraction on the absorption of light, since refraction in the plasma atmosphere causes the light rays to be bent away from the critical surface, where they are most efficiently absorbed (classically as well as ty resonance absorption). The effects of wave swelling on the absorption and the diffraction of light itself both make it difficult to evaluate the effects due to refraction, because changes in the atmospheric scale length which affect the refractive properties of the plasma have, through wave swelling, an even greater effect on the absorption. Similarly, changes in the translation parameter, which affect refraction by adjusting the angle incident rays make with the plasma surface, also cause changes in the diffraction of light around the plasma, which obscure the refractive effects.

We can estimate refractive effects as follows:

From equation 3.30 , the T.E. waves (ignoring resonance absorption) are cutoff at a radius given by

$$
E\left(r_{0}\right)=\ell_{0}\left(\ell_{0}+1\right) /\left(k r_{0}\right)^{2}
$$

which for a linearly varying $E$ gives

$$
\ell_{0}\left(\ell_{0}+1\right)=\left(k r_{0}\right)^{2}\left(r_{0}-r_{c}\right) / \Delta R
$$

We choose $r_{0}$, with account for wave swelling, by estimating the distance from the critical surface at which injerse bremsstrahlung absorption becomes weak. Referring to Figures III, I and III. 2, and using equation 4.2 to calculate $\ell_{0}$, we find: 


\begin{tabular}{|c|c|}
\hline$\underline{\Delta R}(\mu \mathrm{m})$ & $r_{0}$ (estimated) \\
\hline 5 & $r_{c}+\Delta R / 3$ \\
\hline 50 & $r_{c}+\Delta R / 5$ \\
\hline
\end{tabular}

where $\ell_{0}$ is the lowest multipole which does not penetrate the absorption region. Thus, with this simplified analysis, we would predict that an increase in the atmospheric scale length of $5 \mu \mathrm{m}$ to $50 \mu \mathrm{m}$ would cause an increase in the refractive effects by about 15\%. In Figures IV.16 and IV. 17 we observe the effect of increasing the atmospheric scale length on the forward scattered light. This we al so compare with the geometric optics solution, with no absorption. In both cases the forward scattered light is negligibly affected by the change in scale length, while, as a result of wave swelling, the absorption is considerably changed.

It would perhaps be more meaningful to compare plasmas which have the same mass, rather that identical critical radii, since then the plasma with the longer scale length would have a smaller critical radius and therefore intercept a smaller fraction of the light geometrically, lowering its apparant efficiency for absorption. Such a comparison requires a model for the density variation throughout the plasma and we shall not go further than this.

Another way to address the problem of refraction is through the angular deviation from normality of the rays at the plasma boundary. Rays which deviate far from the normal will not reach the critical surface and are not as strongly absorbed, and refraction effects further bend the rays away from normality. He calculate the allowable deviation as shown in Figure IV.18, where from the previous analysis, we use 
for the impact parameter the factor $r_{b}=\ell_{0} / 1$. All rays which pass within $r_{b}$ of the plasma center are acceptable. This gives the following results:

$\begin{array}{ll}\underline{\Delta R}(\mu \mathrm{m}) & \underline{\theta_{b}} \\ 5 & 32^{\circ} \\ 50 & 19^{\circ}\end{array}$

From this point of view, then, the refractive effects appear to be significantly more important as the scale length increases. This is th. case because, for the same $\ell_{0}$, the plasma with a thicker atmosphere has a more restrictive condition on $\theta_{b}$.

The somewhat arbitrary procedure in the choice of $r_{0}$ can be significantly improved by the following analysis: In Figure III.5 and Figure III.7 we plotted the absorption as a function of temperature and multipole, for various plasma configurations. In these plots wave swelling is fully considered, as these results follow from an analytic integration of the Poynting vector over the surface of the plasma. We define $\ell_{b}$ as the multipole for which absorption is $50 \%$ of the $\ell=1$ absorption, for the $\psi$ mode. This insures that a minimum absorption is attained for all light within a specified deviation from normality at the plasma boundary (which is a function of temperature because the value of the multipole $\ell_{b}$ is a function of temperature). (For some data in the following chart, Figures III.5 and III.7 do not extend to sufficiently large $\ell$, in which cases the raw data are not given. The same is true for temperatures which do not appear in these figures.) 


$\begin{array}{llll}\Delta \underline{\Delta R}(\mu \mathrm{m}) & \underline{I}(\mathrm{eV}) & \underline{\ell_{\mathrm{b}}} & \underline{\theta_{\mathrm{b}}} \\ 50 & 60 & 762 & 54^{\circ} \\ 50 & 180 & 606 & 40^{\circ} \\ 50 & 840 & 367 & 23^{\circ} \\ 50 & 4000 & 329 & 20^{\circ} \\ 5 & 60 & 440 & 42^{\circ} \\ 5 & 180 & 346 & 32^{\circ} \\ 5 & 840 & 312 & 28^{\circ} \\ 5 & 4000 & 308 & 28^{\circ}\end{array}$

These results are basically the same as before, but the effect of swelling is more accurately considered. The conclusions on the required normality of incident light rays are in agreement with the commonly stated criteria (NUCKOLLS (1974), p. 4!2).

IV. C. Fields Inside the Plasma

We have described in the previous section the scattering and absorption by a spherical plasma when illuminated by a focused laser beam. Also of interest is the distribution of fields within the plasma, which determines, among other things, the spatial distribution of the energy dissipation in the plasma and the location and strength of various nonlinear interactions.

Solutions of the radial wave equation for the Debye potentials were given in Chapter III, where we derived the standing wave solutions characteristic of the radial dependence, but the field distribution must also include both the angular dependence of the spherical hamonics and the effect of differentiating the potential functions, as given in Table 3.2 . 
We will discuss here the variations of the electric field quantities on spherical surfaces inside the plasma and the radial dependence of the electric fields and the accompanying energy dissipation.

At low temperatures, the largest electric fields in the plasma are transverse, occurring near the critical surface at the peak of the first standing wave. In Figure IV. 19 we plot contours of $\langle E\rangle^{2}$ on the spherical surface at this radius, for $T=60 \mathrm{eV}$. Again, the plasma configuration is $r_{c}=100 \mu \mathrm{m}, \Delta R=5 \mu \mathrm{m}$ and $\mathrm{f} \#=0.5$. As in earlier contour plots, the coordinates are of angle, and the linear plots to the right and above describe the amplitude of the quantity along the corresponding axis. As expected, the transverse fields retain the symmetry of the incident beam. (The quantities $\langle E \phi\rangle$ and $\langle E e\rangle$ separately do not, however). As the temperature is increased, the radial fields due to resonance absorption become important. In Figure IV.20 we plot $-E_{r}>$ on the critical surface, for $T=850 \mathrm{eV}$. The resonance absorption peaks in lobes to the north and south of the beam center, along the vertical axis, and vanishes along the horizontal axis. (In Figure IV.20 notice that the magnitude of the field on the horizontal axis is down by a factor of $10^{14}$.) In this case the transverse fields are approximately equal in magnitude to the radial fields, as seen in Figure IV.21, a plot of $\langle E\rangle^{2}$ on the critical surface. At still higher temperatures, $T=1350 \mathrm{eV}$, the radial fields become more dominant in the plots of $\langle E\rangle^{2}$, as in Figure IV.22.

We can also make contour plots of the cross section of the field distribution, as in Appendix 2, but the scale of the radial variations compared to the plasina size makes it difficult to read the details of 
any such plots. If we employ a very narrow incident beam the interaction with the plasma will occur on a small section of the plasma surface, and we can then blow up a portion of the contour plot and see the transverse and radial variations on the same scale. We do this by calculating the interaction of an $\$ 70.0$ beam with a plasma whose parameters are the same as above. In Figure IV. 23 we show the region of interaction of the beam on the scale of the entire plasma. In Figure IV. 24 are plotted contours of $\langle E\rangle^{2}$ for $\phi=0$, (vertical plane) for the region shown in the previous plot, and for a plasma temperature of $60 \mathrm{eV}$. For this and following plots, the contours are successively diminished by $50 \%$ from the next higher contcur. In this manner, contours many times smaller than the peak value can be plo'ted an a single pass. Evident in Figure IV. 24 are the standing waves, whose peaks are smaller moving away from the critical surface (which is at $\left.k_{\rho}=628.3\right)$. Also note the transverse behavior, which falls off on the spherical surfaces moving away from the central axis. As we pointed out, this result is for an f 10 focusing system; the effects for faster focusing are similar but not so easily displayed to scale on a contour plot. In this plot, the linear graph to the right plots $\langle E\rangle^{2}$ along the axis $y=0$, and the one on the top is of $\langle E\rangle^{2}$ along the outemost radial used in the contour plot. Figure IV.25 is of $\langle\mathbf{E}\rangle^{2}$ in the plane $\phi=\pi / 2$. It is virtually identical to the previous plot, because at this temperature all absorption is classical and polarization effects not significant.

Now we consider $T=180 \mathrm{eV}$, in figures IV. 26 and IV.27. The decrease in collision frequency has sharpened the standing waves, and 
the effect of resonance absorption on $\langle E\rangle^{2}$ is just beginning to be seen. Natice that there is no effect on the axis of the beam, but contours away from the axis are beginning to distort at the critical surface. The plot along the radial (at $k y \approx 55$.) also shows the influence of the plasma waves. As expected, there is no effect at $\phi=\pi / 2$.

Finally at $T=850 \mathrm{eV}$, in Figures $I V .28$ and $I V .29$, we see strong resonance absorption fields at the critical surface both in the linear plot along the outer radial and the heavy black line in the resonance absorption region, representing the presence of a large number of contour lines rising very steeply. Again, along the axis and at $\phi=\pi / 2$ no effects of resonance absorption are seen.

We can determine the energy deposition of the beam in the following manner: the energy deposition at a point in the plasma is proportional to $v(r)<E>^{2}$ so on a sphere of constant radius, the variation of the energy dissipated conforms to the plots of $\langle E\rangle^{2}$ already discussed. These results showed how, for low temperatures, the energy deposition has the symmetry of the incident beam, and as the temperatures increase, asymmetrical absorption due to plasma waves (resonance absorption) becomes dominant.

In the radial direction we can simply determine the rate of energy dissipation inside a given radius by integrating the norma 7 Poynting vector over the sphere described by that radius. These plots, of energy deposition inside a specified radius, are shown for an atmospheric thickness of $5 \mu \mathrm{m}$ and $k \rho$ of 0 and 100 in Figures IV. 30 and IV.31. 
We see the effects of the standing waves in the added fraction of absorption occurring as we advance across each successive wave. At very high temperatures, virtually all the absorption is due to resonance absorption, which occurs at the critical surface. This is observed in Figure IV.30, which shows substantial resonance absorption in the near step funcition rise of absorption at $r_{c}$.

As the temperature is lowered the absorption occurs further from the critical surface and the resonance absorption is lowered in magnitude, because the wave is classically attenuated before it reaches the critical surface.

IV. D. Self Consistent Analysis of Approximations

Thus far in Chapter IV we have tacitly assummed that our solutions are valid for a wide range of input parameters. In fact, breakdown of the cold plasma and linearizing approximations places limits on the incident beam power and plasma temperatures for which the equations can be used. In this section we will determine the beam power and temperature for which the various approximations fail.

First, we consider in more detail the cold plasma approximation. We have ignored terms of the order

$$
p=\frac{\frac{r \theta}{m c^{2}}|\nabla \nabla \cdot E|}{k^{2 v} \frac{v}{\omega}|E|}
$$

This term is largest at the critical surface where we can approximate

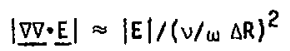


So we consider the quantity

$$
p=\frac{y \theta}{m c^{2}} /\left[(k \Delta R)^{2}(v / \omega)^{3}\right]
$$

Using equation 3.4 , and $\gamma=1$.

$$
p=\left(\frac{1}{k \Delta \mathrm{R}}\right)^{2}\left(\frac{T}{62.7}\right)^{11 / 2}
$$

where $T$ is the plasma temperature in eV

Then, for $p=1$

$$
T_{p W}=62.7(k \Delta R)^{4 / 11}
$$

which is the temperature at which plasma wave effects are equivalent to collisionai effects at the critical surface.

Returning to equation 4.5 , we define an effective collision frequency due to plasma waves,

$$
\frac{v_{\mathrm{pw}}}{\omega}=\left[\frac{\gamma \theta}{\mathrm{mc}^{2}(k \Delta R)^{2}} \frac{1}{(1 / 3}\right.
$$

which is the same as GINZBURG (1964) p.227.

In the following plats of Power versus Temperaiure, we denate results for $T>T_{p w}$ with dashed lines.

Our analysis of the linearizing approximations proceeds as follows: We choose a laser-lens-plasma configuration for analysis, and for a suecified temperature, calculate the fields inside the plasma. Then, looking at the largest field amplitude, plasma wave amplitude, and radial gradient of $\left\langle\mathrm{E}_{r}\right\rangle^{2}$ we determine by scaling the incident beam 
power, the power for which the following conditions hold:

$$
\begin{aligned}
& P_{\text {or }}: v_{\text {or }}=v_{\text {th }} \\
& P_{\text {ot }}: v_{\text {ot }}=v_{\text {th }} \\
& P_{n}: n_{1}=n_{0} \\
& P_{\nabla E^{2}}: \nabla\left(\frac{E^{2}}{8 \pi}\right)=\nabla n k T
\end{aligned}
$$

Where $v_{\text {or }}=$ escillation velocity of electrons due to radial electric field.

$$
v_{\mathrm{ot}}=\text { oscillation velocity of electrons due to transverse }
$$

electric fields

$$
v_{\text {th }}=\text { thermal velocity of electrons }
$$

The last condition above for $P_{\nabla E^{2}}$ (as it turns out, the most easily violated) is the situation in which self steepening of the density gradient will occur (DEGROOT and TULL (1975)). Here the radial component of the ponderamotive force due to the plasma wave is as large as the force due to the pressure gradient, and being asymmetric, steepens the density gradient more in some places than in others. The subsequent breakdown of spherical symetry invalidates our solutions. This condition does not apply for times short compared to the time it takes the ions to move, which is on the order of several hundred light periods, $\approx 10^{-12} \mathrm{~s}$.

These four quantities are plotted as a function of temperature in Figures IV.32-35 for two atmospheric thicknesses and for two translations. The translations are chosen to minimize and maximize the 
effects of resonance absorption, to establish an upper and a lower limit to the effect of the plasma waves.

For minimum resonance absorption, we consider the case in which the beam focus and the plasma center coincide. The maximum resonance absorption occurs for a translation slightly off center and we choose a positive $\rho$, so that the maximum area is illuminated by the beam. fris other plasma-laser positioning, which necessarily involves an intermediate amount of power being resonantly absorbed, will produce fields such that the Power versus Temperature curves 1 ie roughly between the two given here. (For the situation in which the laser is focused one or two radii behind the center of the plasma, the incident power will be spread over a larger area of the sphere, in which case these results may be somewhat pessimistic. We cannot make that particular interesting calculation because of computer storage limitations).

We also consider two geometries, $\Delta \dot{R}=50 \mu \mathrm{m}$ and $\Delta R=5 \mu \mathrm{m}$ with $r_{c}=100 \mu m$. All results are for $f \# 0.5$.

The results are in Figures IV.32 through IV.35.

At minimum resonance absorption, the transverse electron oscillation velocity exceeds the electron thermal velocity at $10^{11}-10^{12}$ $\mathrm{W}$ for low plasma temperatures. As $\mathrm{T}$ increases, the thermal velocity grows faster than the oscillation velocity but the electric field gradients at the critical surface grow faster still. The weak resonance absorption for the minimum resonance absorption calculation is still ultimately responsible for the large field gradient which limits these solutions, for $T$ i $300 \mathrm{eV}$. 
When resonance absorption is maximized, $P_{\nabla E} 2$ limits the solutions at a11 temperatures.

The linearization approximations given by $P_{n}$ and $P_{\text {or }}$ in no instance limit our solutions.

In our solutions, for $T>T_{p w}$, the amplitude of the plasma wave scales as $\left(\frac{v}{w}\right)^{-1}$, and the radial gradient of $\left\langle E_{r}\right\rangle^{2}$ as $\left(\frac{v}{w}\right)^{-3}$; in fact, these numbers should be much smaller, when correctly treated without the cald plasma approximation, because the effect of the temperature dependent term is to limit the size of the electrostatic wave at the critical surface. In Figure IV. 36 ve have plotted the power versus temperature curve when the plasma wave effects are approximately included by using $u_{\mathrm{pw}}$ for $v$ in calculating $\nabla E^{2}$. As $v_{\mathrm{pw}}$ increases with $T, \nabla E^{2}$ decreases so the power required to self steepen increases. The difference between this curve and the calculated and approximate results ignoring plasma waves is large and any analysis in which the magnitude of the fields or their derivatives near the critical surface is important should be used with caution. In Chapter $V$ we discuss methods for more accurately considering the plasma wave effects.

We have also studied other f\#'s and plasma sizes. The ponderamotive force increaces as the intensity which itself scales as $r_{c}{ }^{-2}$ (when we focus the laser beam such that a large surface area is being irradiated). Therefore the self steepening force is decreased by $10^{2}$ for a $1000 \mu \mathrm{m}$ radiur plasma. The fields scale as $I^{1 / 2}$ so for a $1000 \mu m$ plasma, $P_{o r}, P_{o t}$, and $P_{n}$ increase a factor of 10 . In this situation the linear theory is valid for present day laser fusion sources 
$\left(\sqrt{3} 10^{12} \mathrm{~W}\right)$ and for plasmas colder than 200-500 eV. The effect of changing $f \#$ is easily predictable since a larger f\# increases the intensity at the critical surface for the same amount of incident power, and thereby lowers the threshold for the nonlinear effects. 


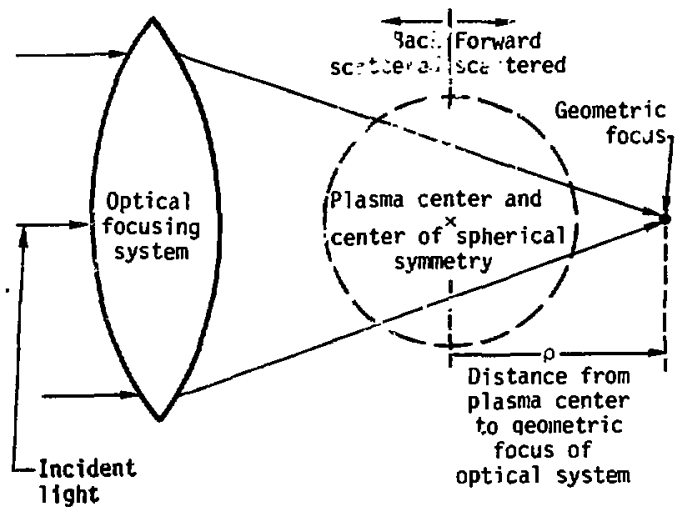

Figure IV. 1 Definition of $\rho$, distance from plasma center to geometric focus of optical system. 


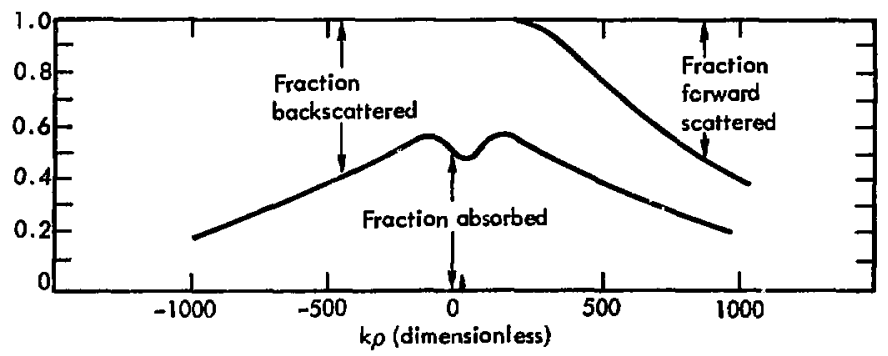

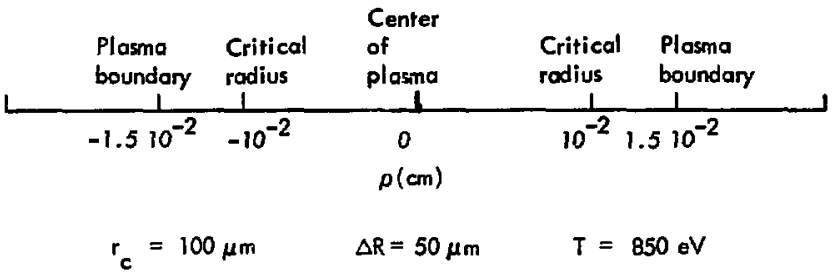

Figure IV.2 Light scattered and absorbed versus position of laser focus, $f \#=0.5$. 

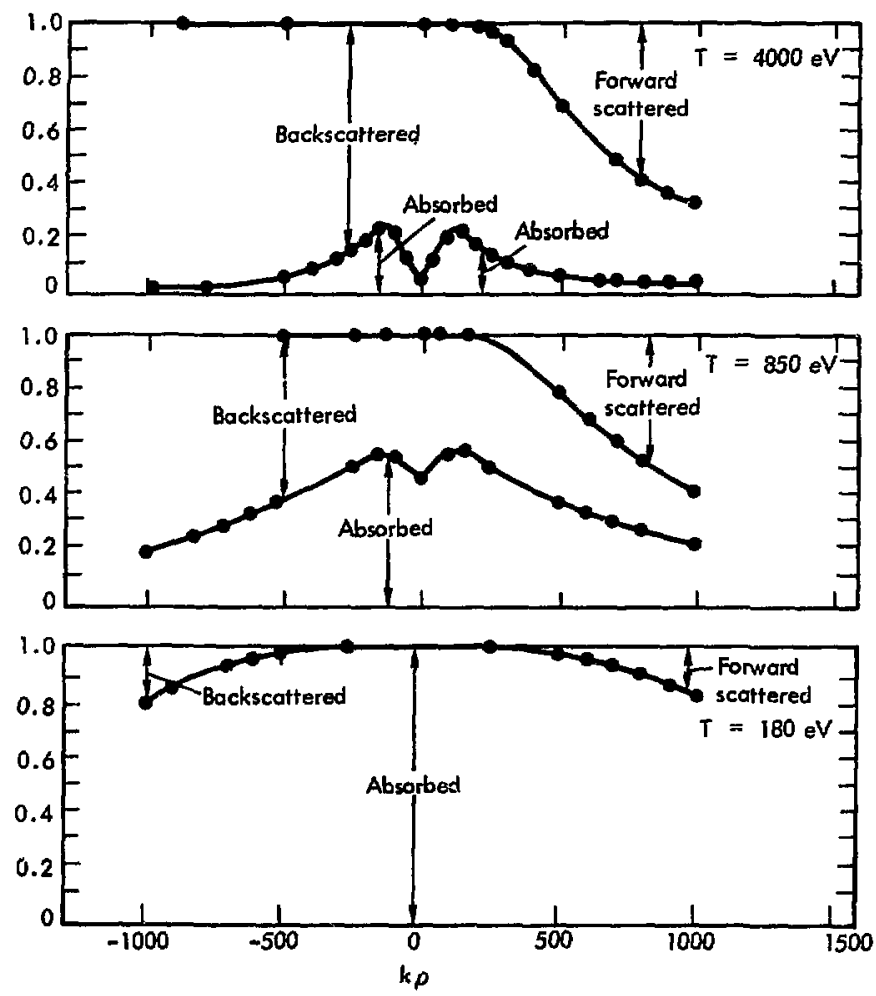

Figure IV.3 Light scattered and absorbed versus position of laser focus, $f \#=0.5, r_{c}=100 \mu \mathrm{m}, \mathrm{AR}=$ $50 \mathrm{\mu m}$, temperature $=4000,850$ and $180 \mathrm{eV}$. 

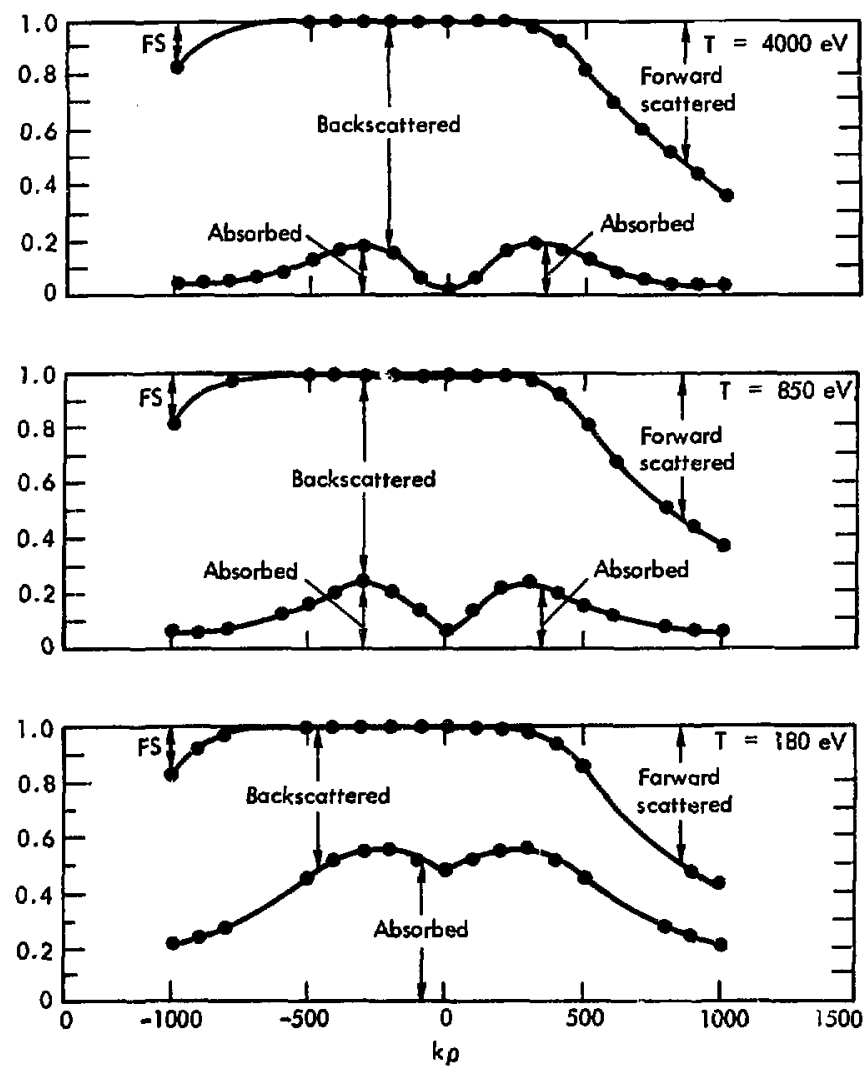

Figure IV.4 Light scattered and absorbed versus position of laser focus, $f \|=0.5, r_{c}=100 \mu \mathrm{m}, A R=5 \mu \mathrm{m}$, temperature $=4000,850$ and $180 \mathrm{eV}$. 

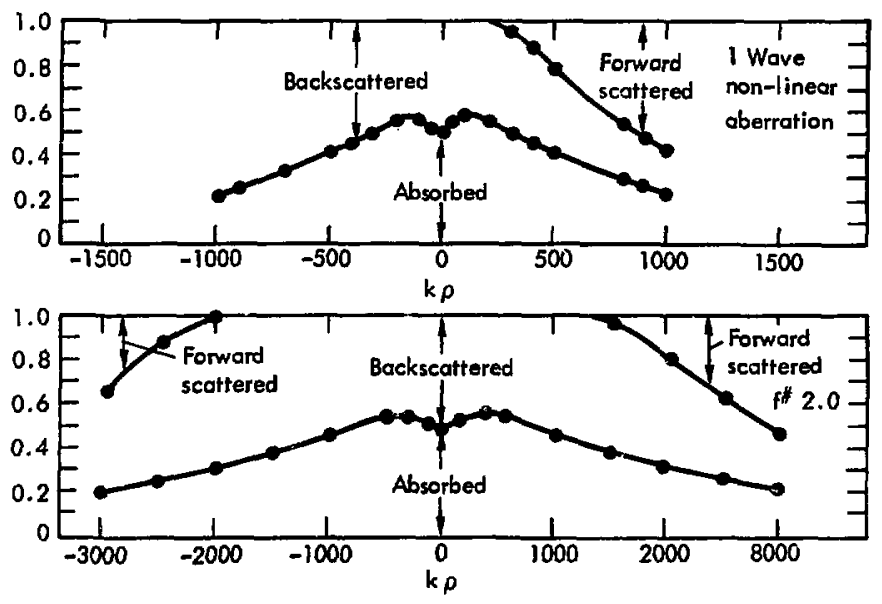

Figure IV.5 Light scattered and absorbed versus position of laser focus, $r_{c}=100 \mu \mathrm{m}, \Delta R=50 \mathrm{\mu m}, T=$ $850 \mathrm{eV}$. (a) $f \|^{c}=0.5, w=1$ wave (b) $f \#=2.0$, $w=0$. 

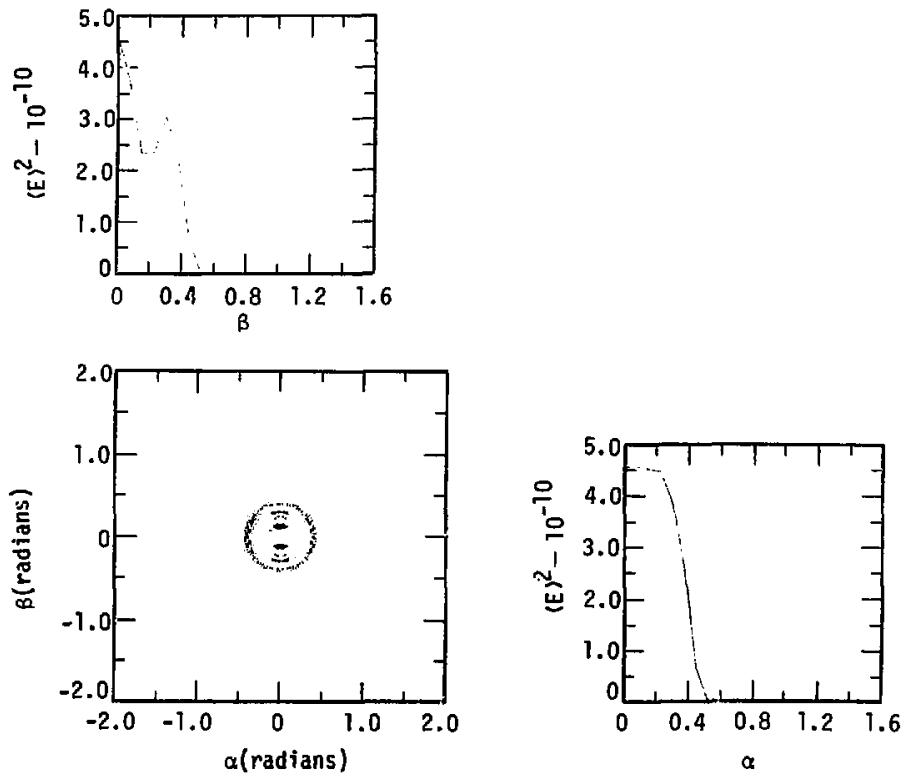

Figure IV, 6 Backscattered light distribution, $k \rho=-500$. 

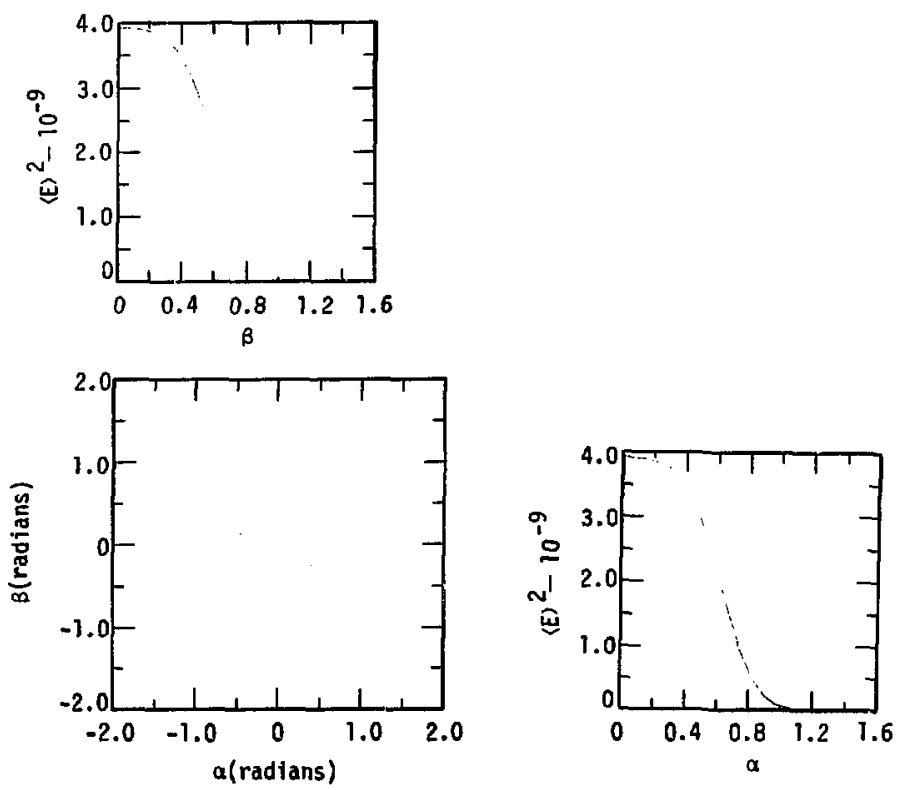

Figure IV.7 Backscattered light distribution, $k \rho=0$ 

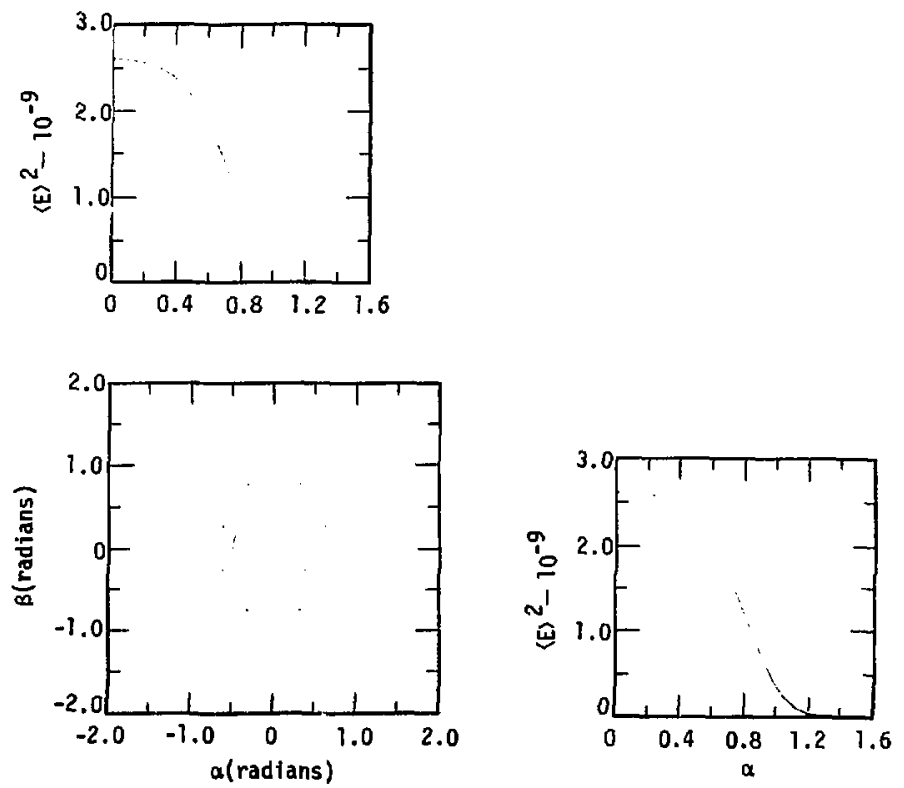

Figure IV. 8 Backscattered light distribution, $k \rho=100$. 

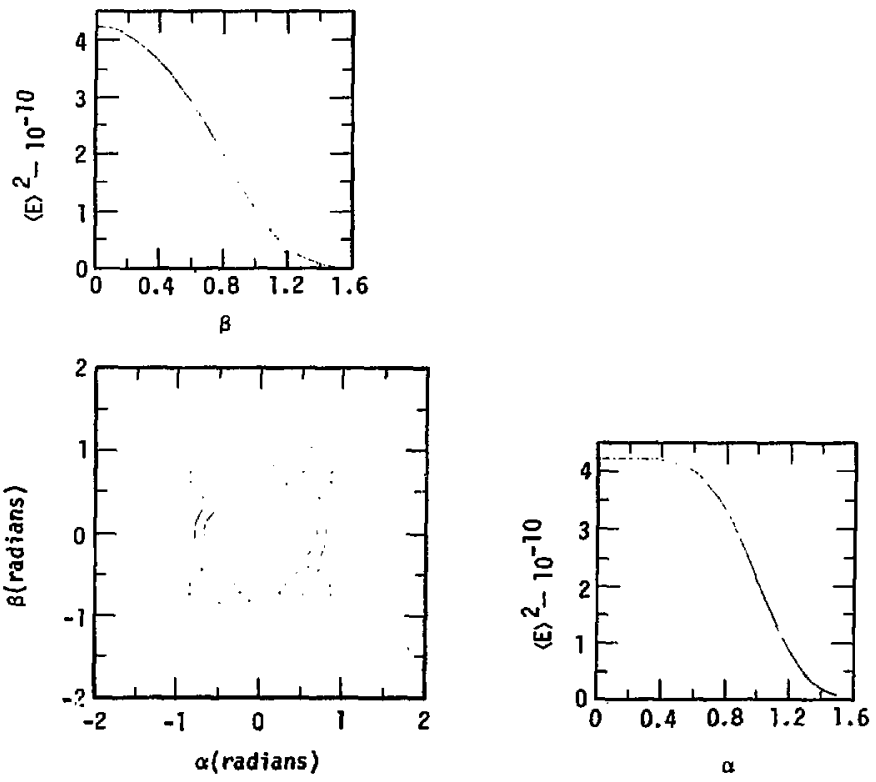

Figure IV.9 Backscattered light distribution, $k \rho=200$. 

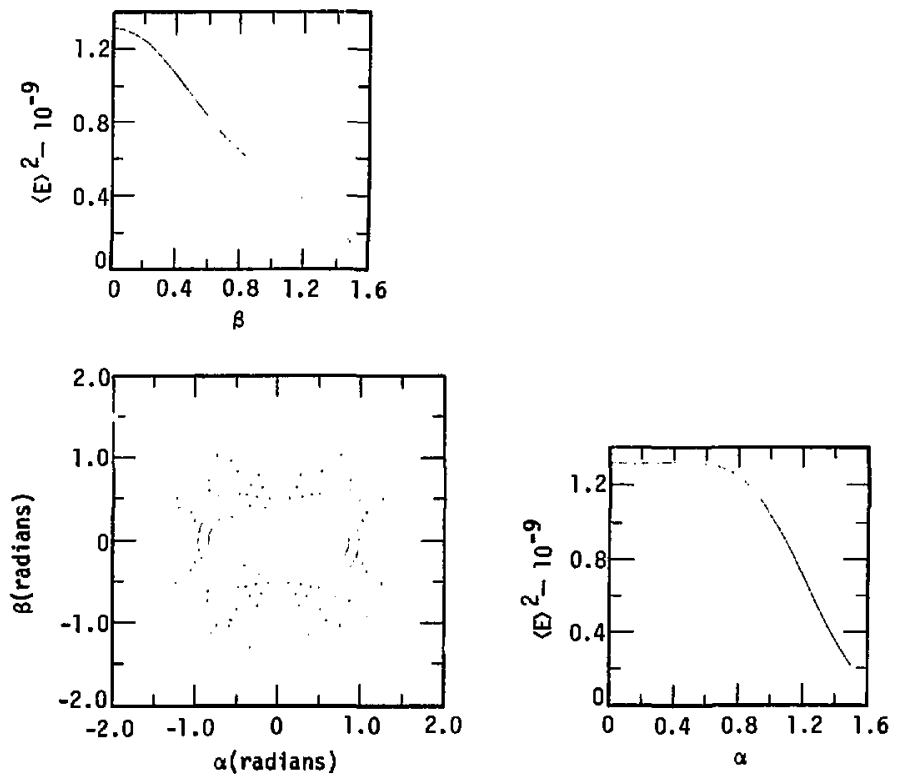

Figure IV.10 Backscattered light distribution, $k_{p}=300$. 

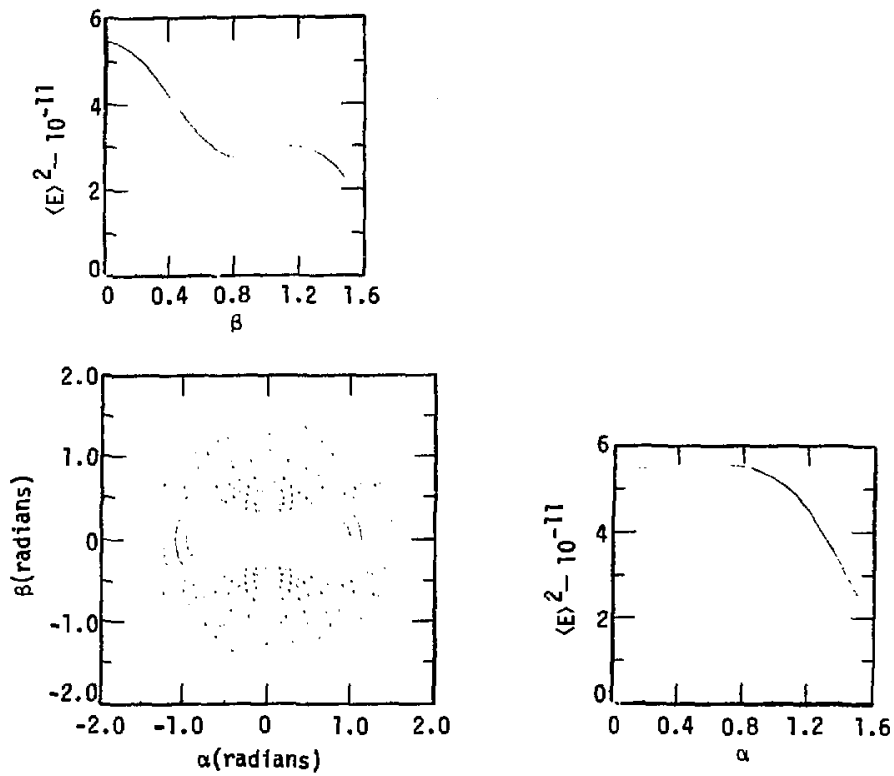

Figure IX.11 Backscattered light distribution, $k \rho=400$. 

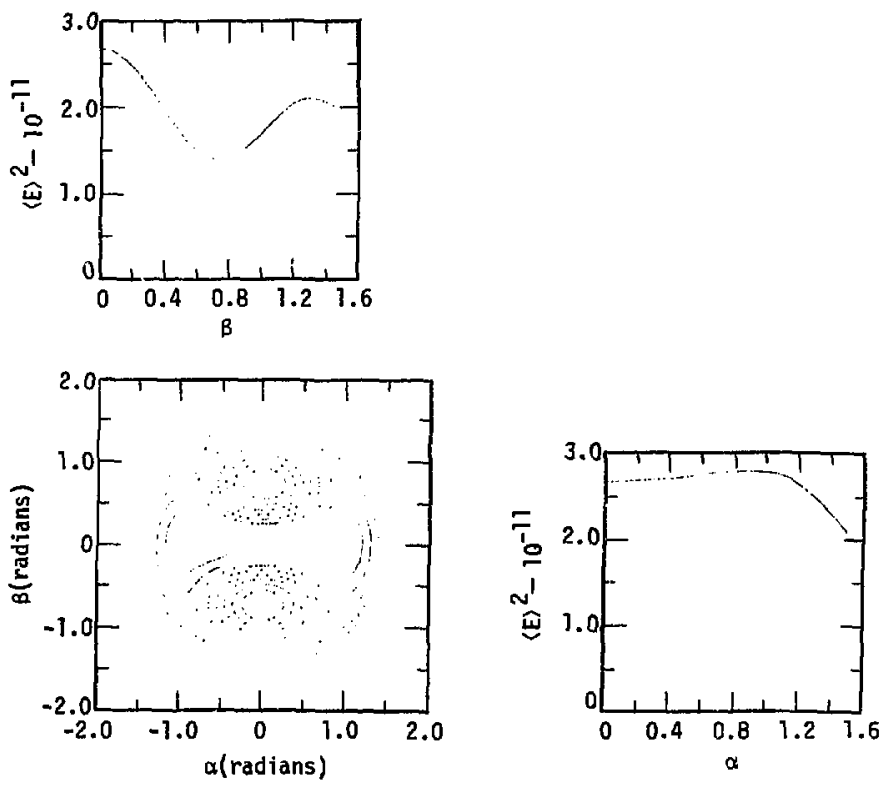

Figure IV.12 Backscattered light distribution, $k_{0}=500$. 

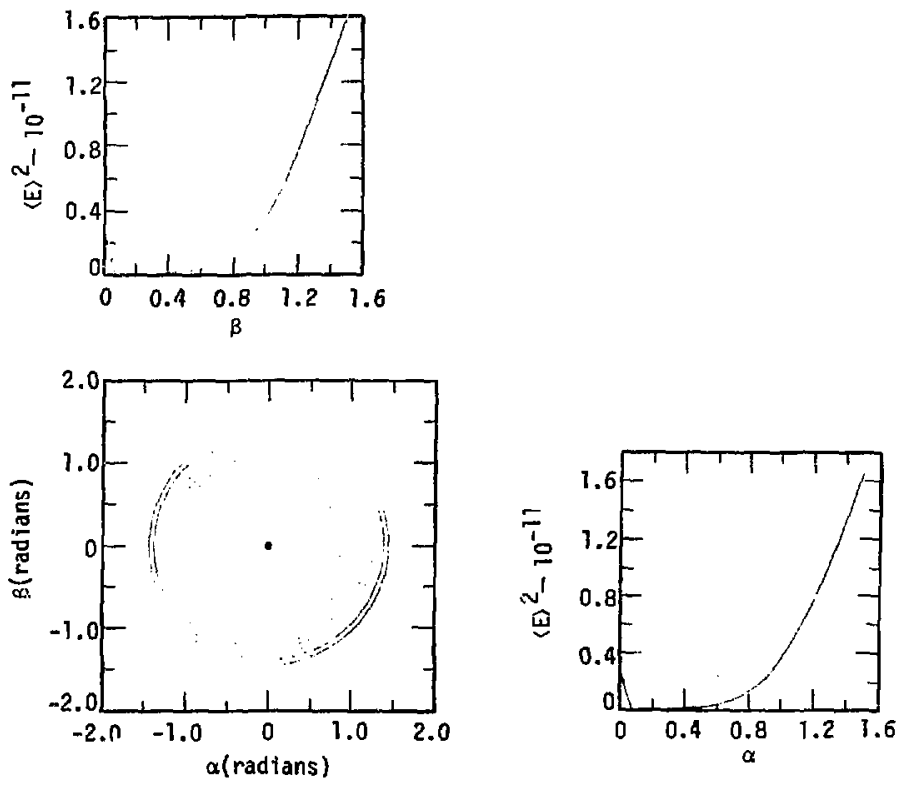

Figure IV.13 Forward scattered Tight distribution, $k \rho=500$. 

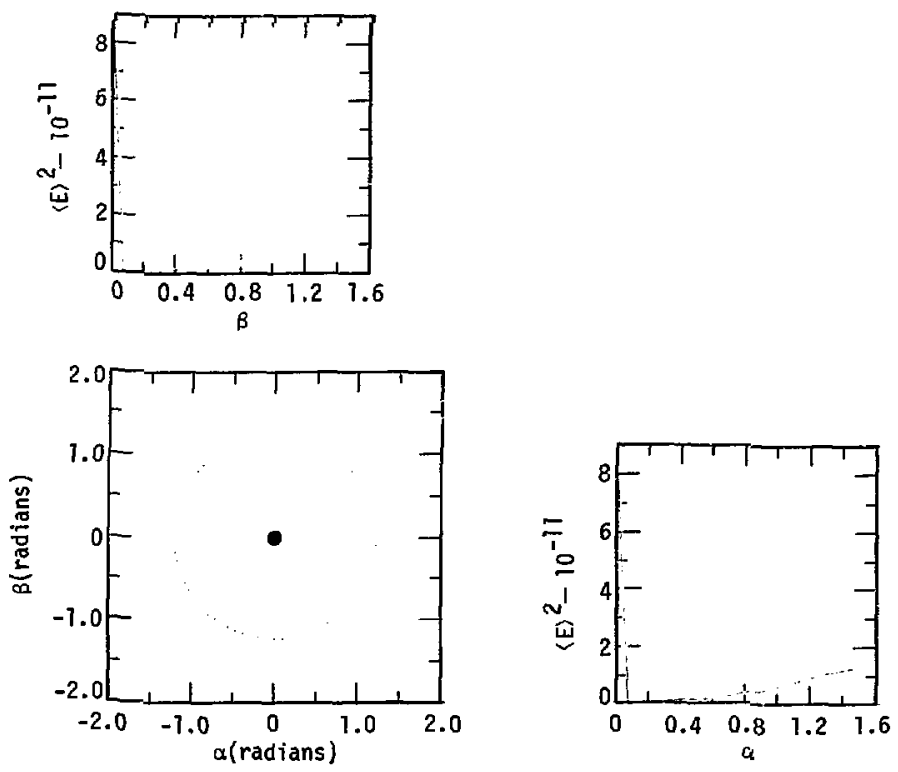

Figure IV.14 Forward scattered light distribution, $k p=600$. 


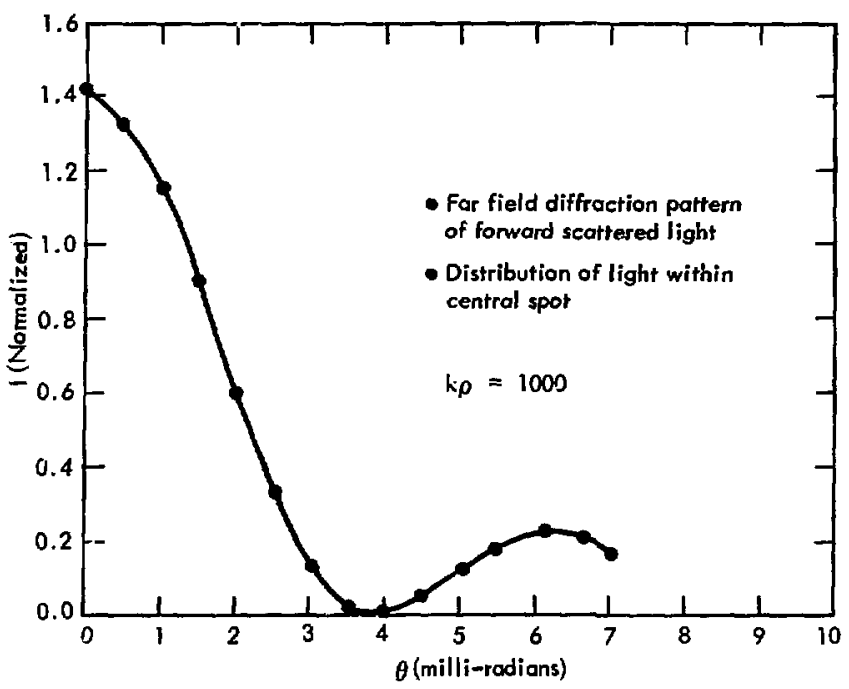

Figure IV.15 forward scattered light distribution, variation in central spot, $k \rho=1000$. 
Absorption and scattering for $\Delta R=50 \mu m, 5 \mu m$ and geometrical solution with no absorption

(geometrica? solution is given by.....) $\quad T=850 \mathrm{eV}$

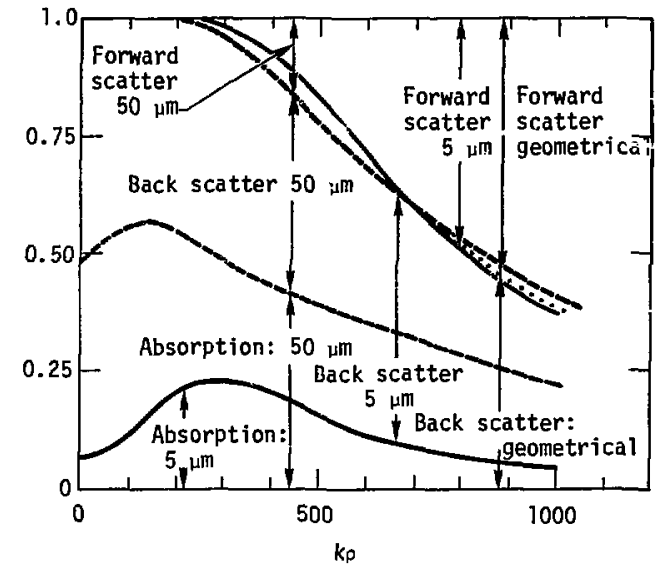

Figure IV.16 Absorption and forward and backscattered light versus $k \rho$ for $\Delta R=50 \mu \mathrm{m}$ and $5 \mu \mathrm{m}$ and geometrical solution, $T=850 \mathrm{ev}$. 


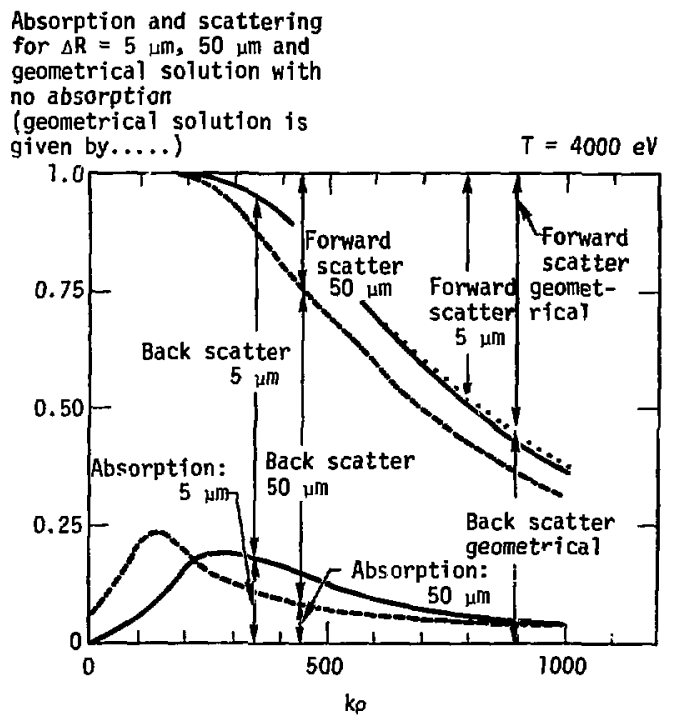

Figure IV. 17 Absorption and forward and backscattered 1 ight versus $k p$ for $\Delta R=50 \mu m$ and $5 \mu m$ and geometrical solution, $T=4000 \mathrm{eV}$. 


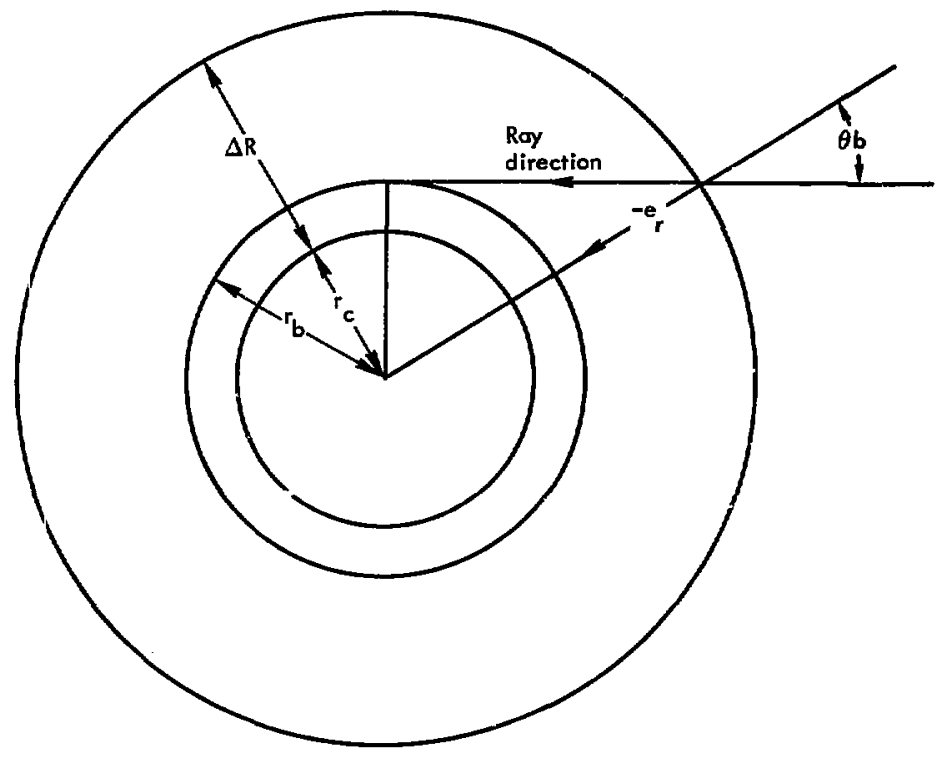

Definition of $\theta_{b}$, allowable deviotion from normality of incident ray

$$
\theta_{b}=\sin ^{-1}\left(\frac{r_{b}}{r_{c}+\Delta R}\right)
$$

Figure IV.18 Illustration of the quantities

$r_{c}$ : radius of critical surface,

$\Delta \mathbb{R}:$ atomospheric thickness

$r_{b}$ : impact parameter

$\theta_{b}$ : ray deviation from nomality 

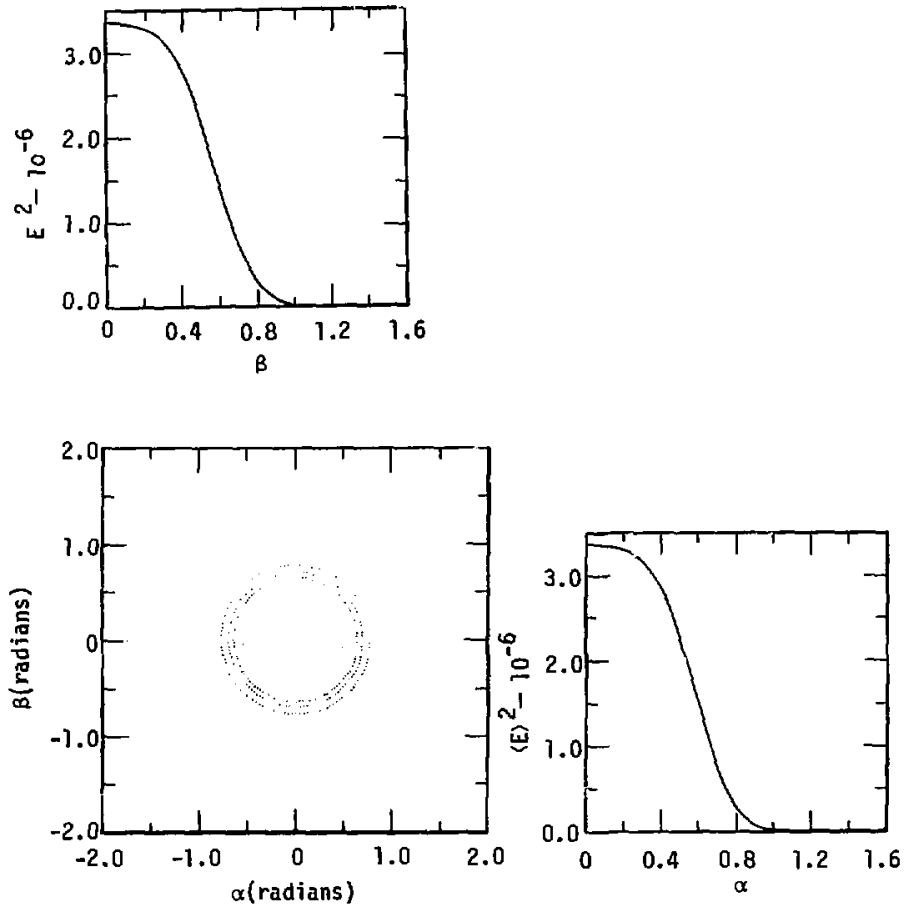

Figure IV.19 $E>^{2}$ an spherical surface at peak of largest standing wave, $r_{c}=100 \mu \mathrm{m}, \Delta R=5 \mu \mathrm{m}, f \#=$ $0.5, T=60 \mathrm{eV}$. 

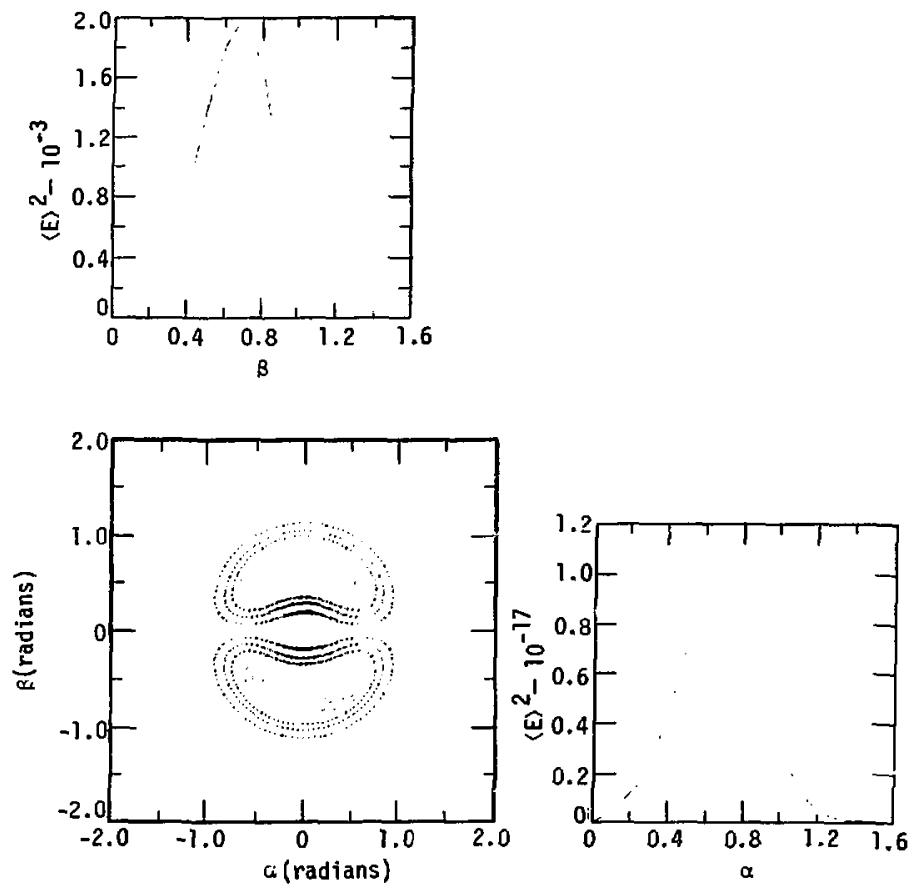

Figure IV.20 $\left\langle E_{r}\right\rangle$ on critical surface, $r_{c}=100 \mu \mathrm{m}, \Delta R=$ $\sim \quad 5 \mathrm{jm}, f \mathrm{ft}=0.5, T=850 \mathrm{eV}$. 

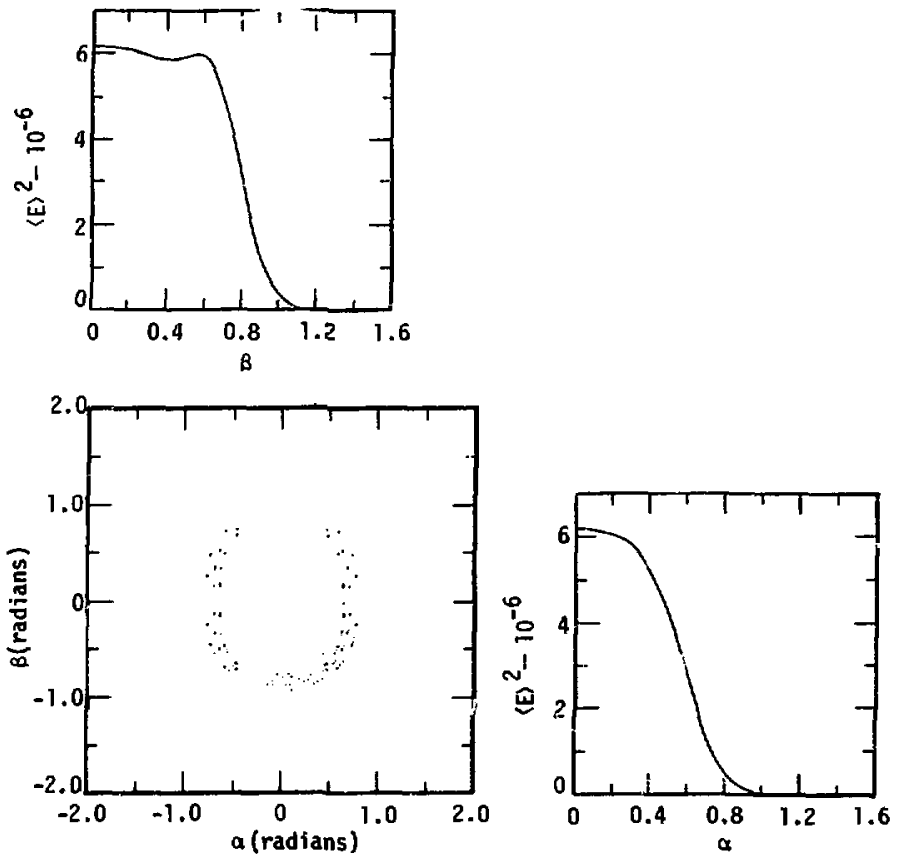
Figure IV.21 $E\rangle^{2}$ on critical surface, $r_{c}=100 \mu \mathrm{m}, \Delta R=$
$5 \mu \mathrm{m}, f \mathrm{f}=0.5, T=850 \mathrm{ev}$. 

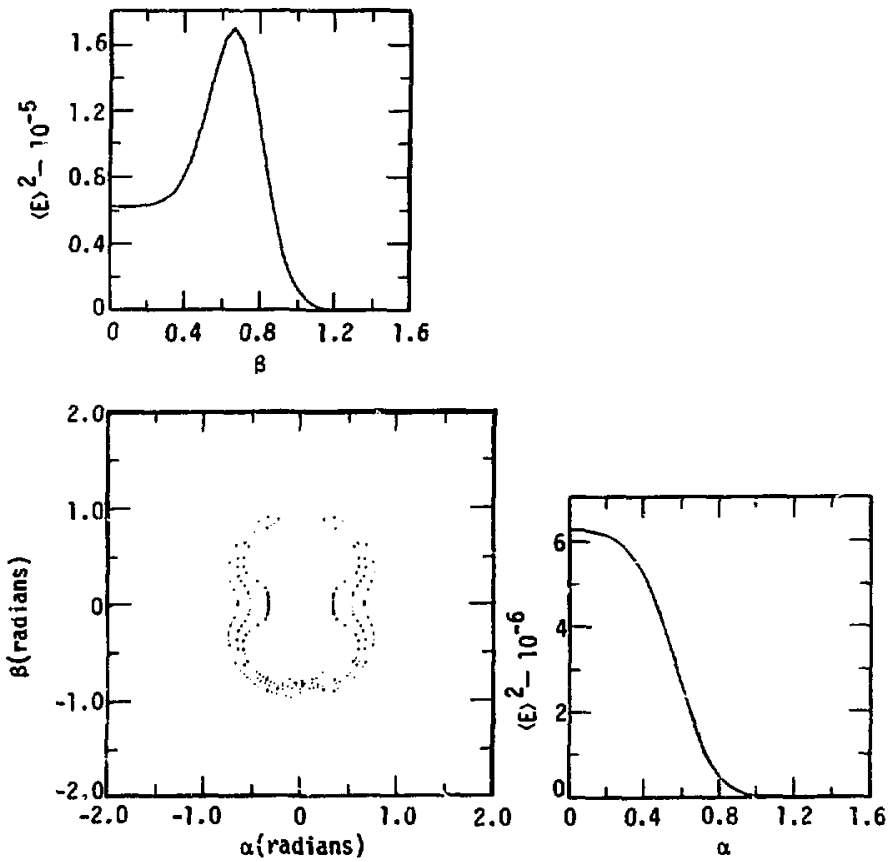

Figure IV.22 $E^{2}$ on critical surface, $r_{C}=100 \mu \mathrm{m}, \Delta R=$ $5 \mathrm{~km}, \mathrm{fH}=0.5, \mathrm{~T}=1350 \mathrm{eV}$. 


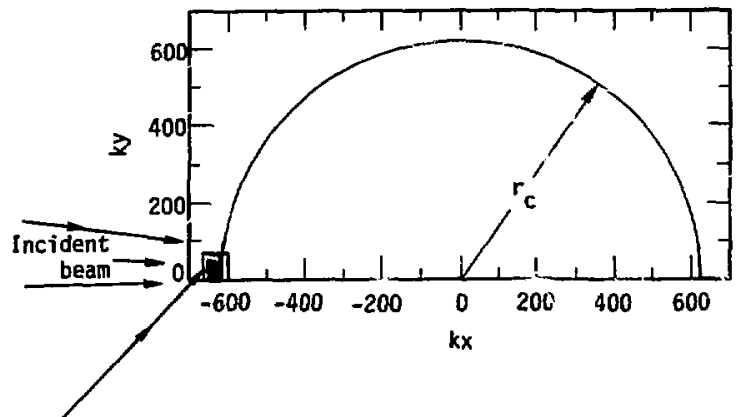

$\angle$ Region blown up in Figures IV.24 through IV.29.

Figure IV.23 Region of beam interaction blown up $;,$ following plots: $f \#=10, r_{c}=100 \mu \mathrm{m}, \Delta R=5 \mu \mathrm{m}$. 

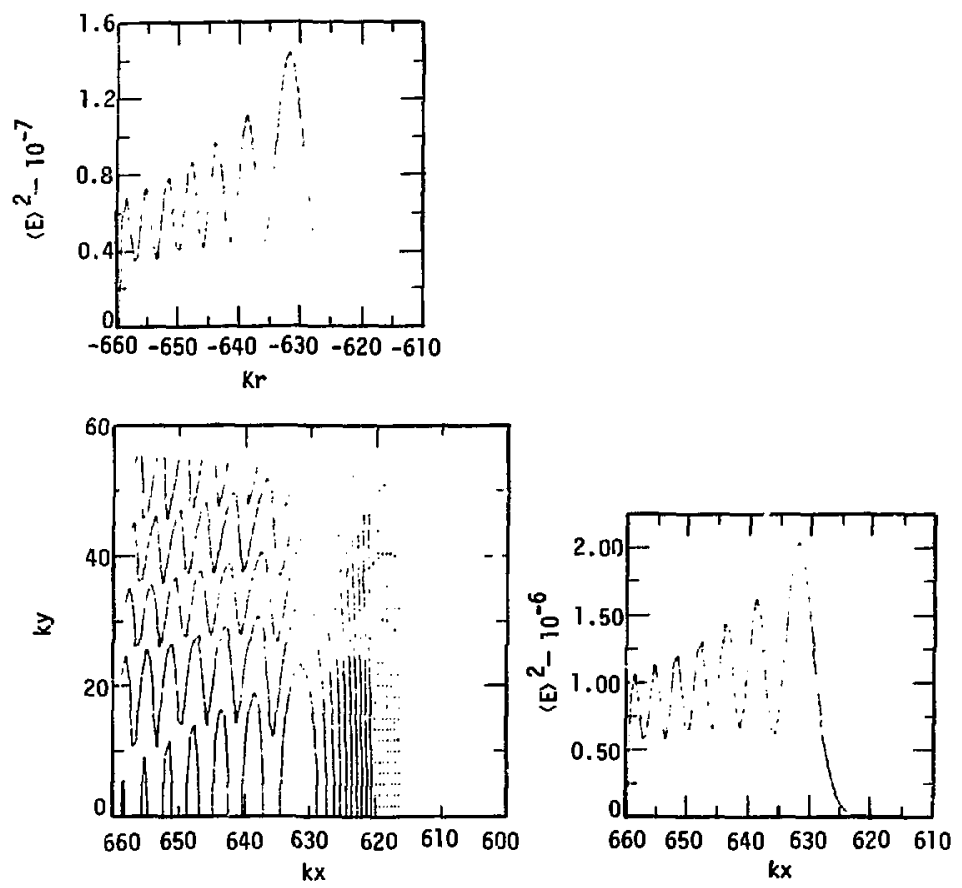

Figure IV.24 $\angle\rangle^{2}$ in plane $\phi=0, f \#=10, r_{C}=100 \mu \pi_{,}$ $\Delta R=5 \mu \mathrm{m}, T=60 \mathrm{eV}$. 

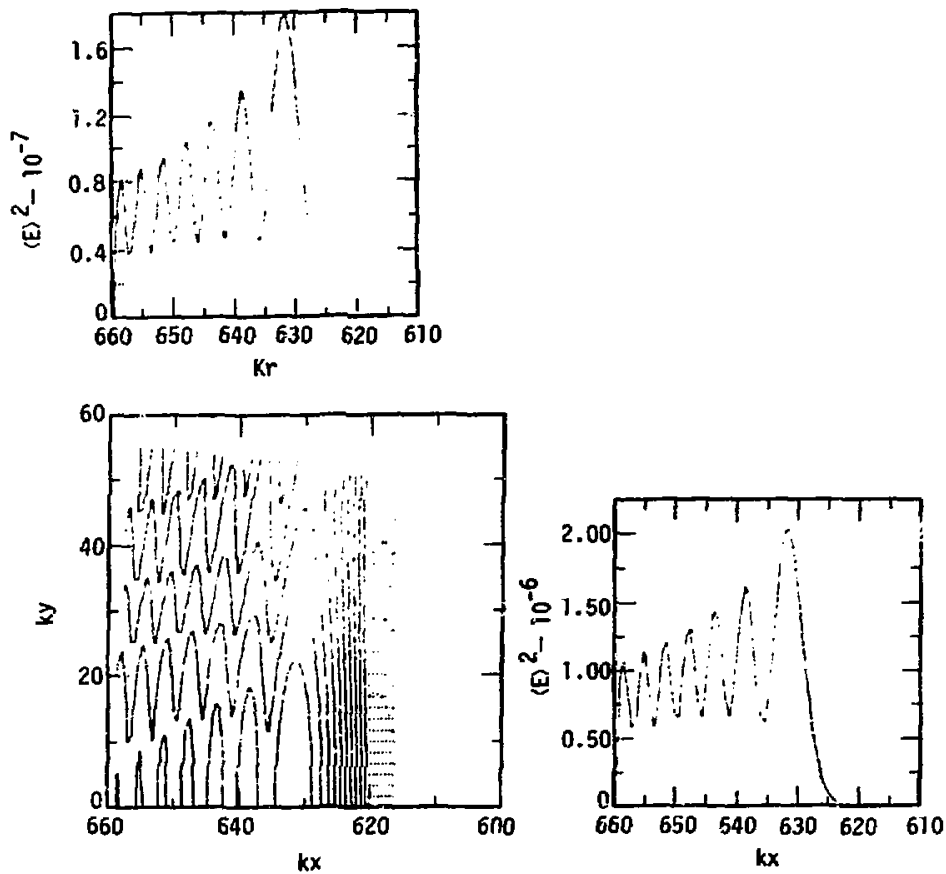

Figure IV.25 $E\rangle^{2}$ in plane $\phi=\pi / 2, f^{H}=10, r_{C}=100 \mu \mathrm{m}$, $\Delta R=5 \mu \mathrm{m}, T=60 \mathrm{eV}$. 

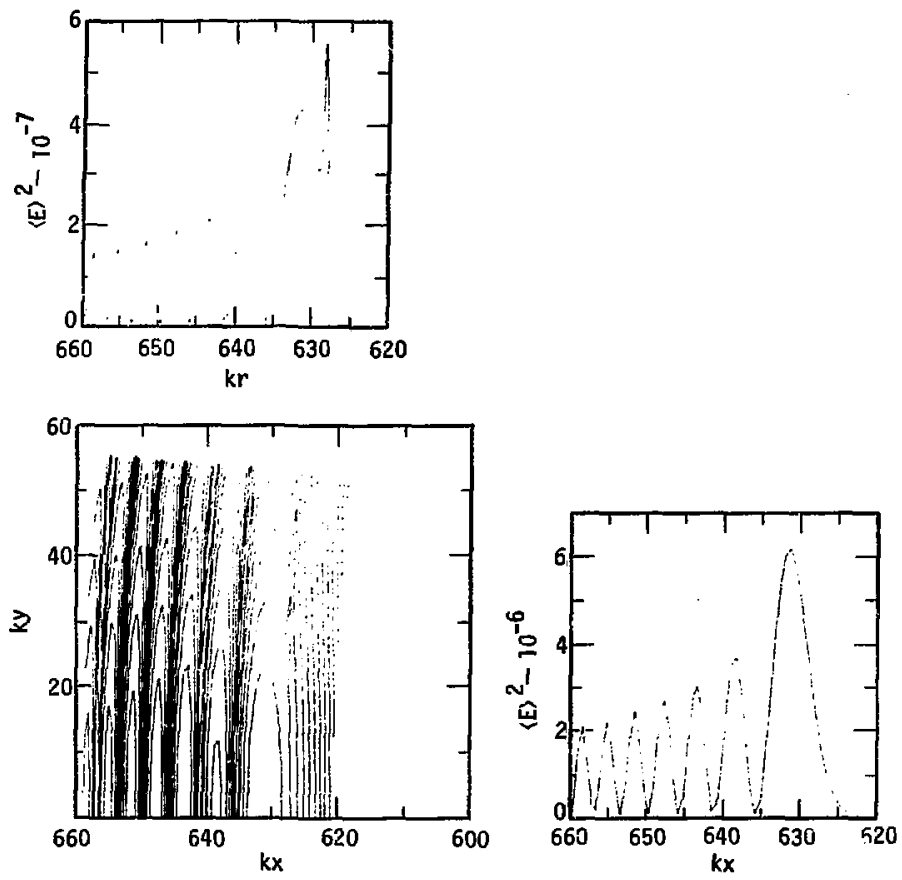

Fjgure IV.26 $\varangle>^{2}$ in plane $\phi=0, f \#=10, r_{C}=100 \mu \mathrm{m}$, $A R=5 \mu \mathrm{m}, T=180 \mathrm{eV}$. 

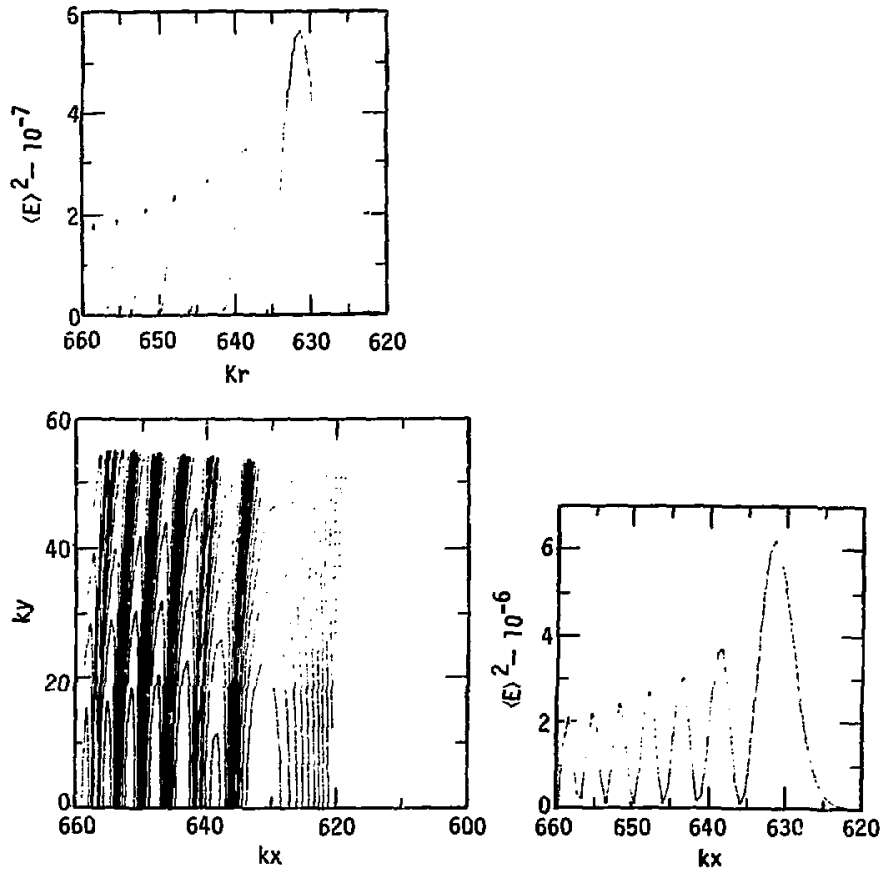

Figure IV.27 $\langle E\rangle^{2}$ in plane $\phi=\pi / 2, f \#=10, r_{c}=100 \mathrm{\mu m}$, $\Delta R=5 \mu \mathrm{m}, T=180 \mathrm{eV}$. 

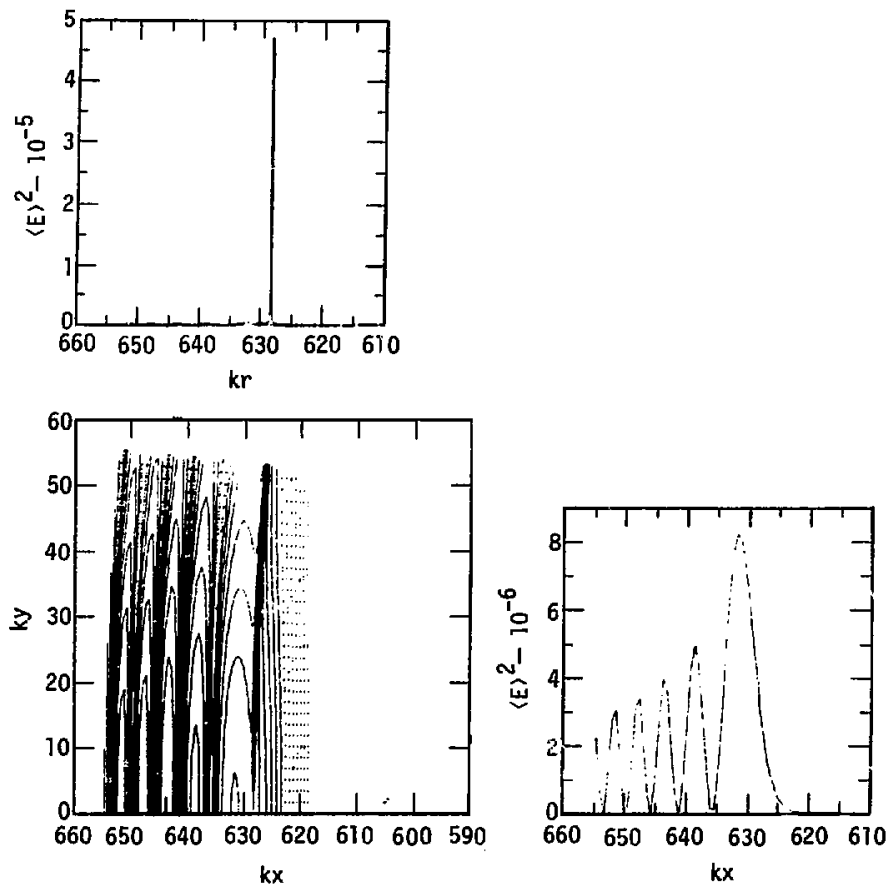

Figure IV.28 $E>^{2}$ in plane $\phi=0, f=10, r_{C}=100 \mu \mathrm{m}$, $\Delta R=5 \mu \mathrm{m}, \mathrm{T}=\mathrm{B} 50 \mathrm{eV}$. 

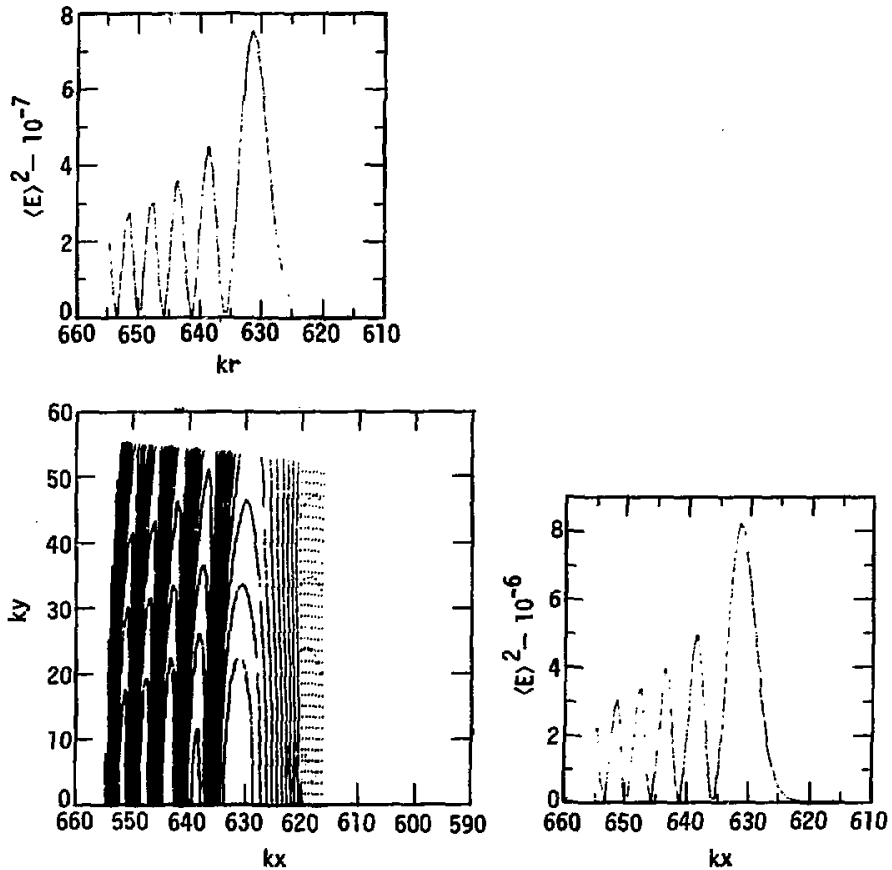

Figure IV.29 $E>^{2}$ in plane $\phi=\pi / 2, f \#=10, r_{c}=100 \mu \mathrm{m}$, $\Delta R=5 \mu \mathrm{m}, T=850 \mathrm{eV}$. 


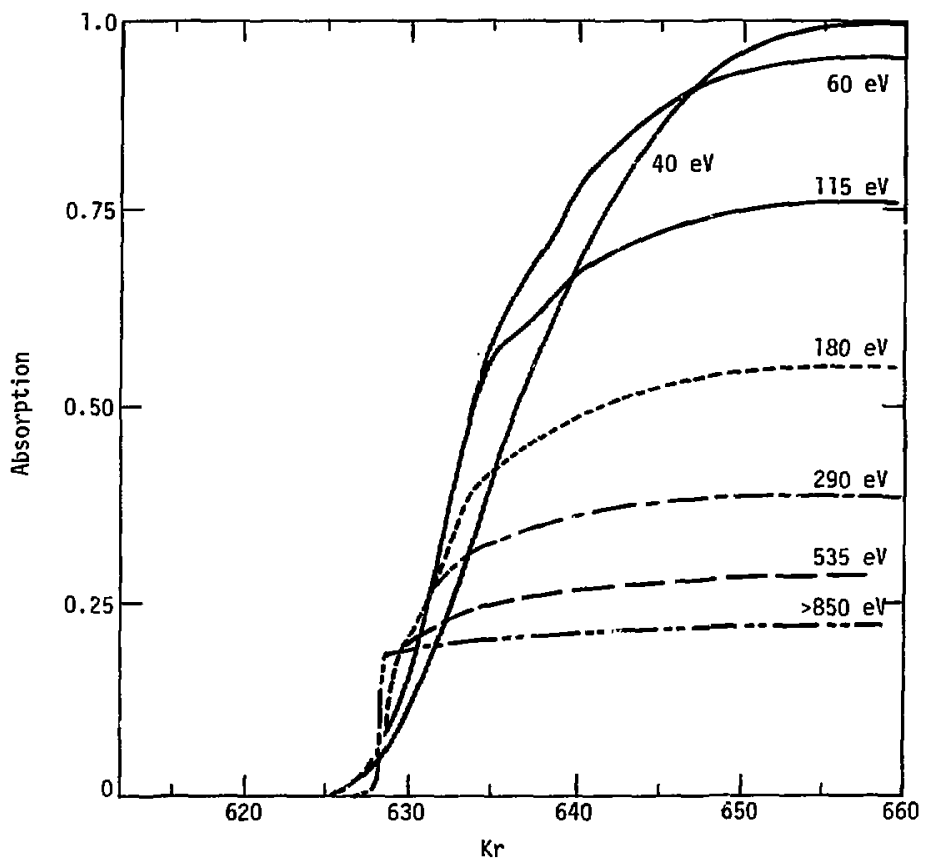

Figure IV.30 Radial dependence of the absorption for various temperatures, $k \rho=100, r_{c}=100 \mu m$, $\Delta R=5 \mu \mathrm{m}$. 
105

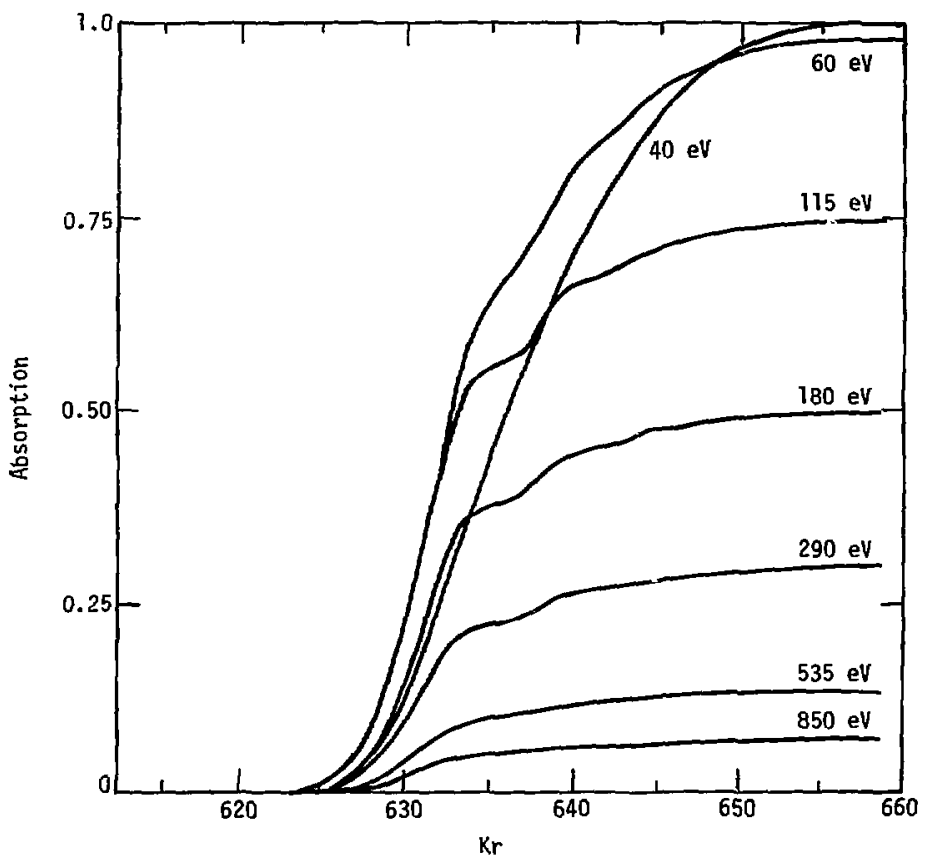

Figure IV.31 Radial dependence of the absorption for various temperatures, $k \rho=0, r_{c}=100 \mu \mathrm{m}$, $\Delta R=5 \mathrm{um}$. 


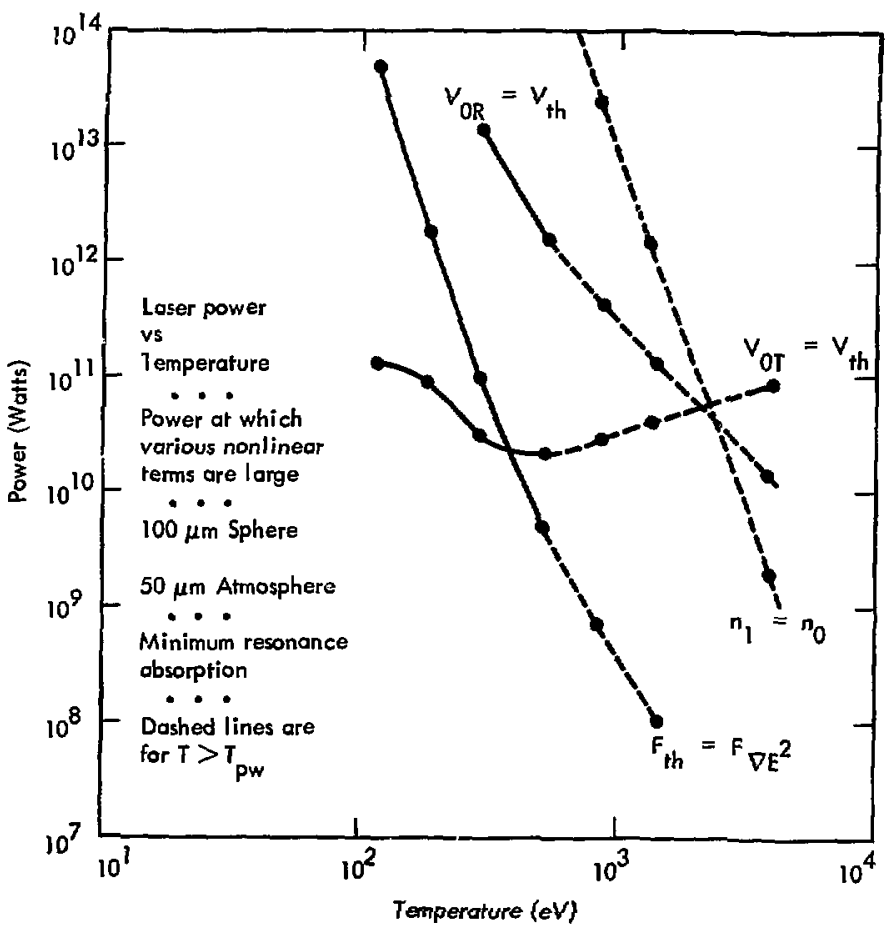

:igure IV.32 Laser power versus temperature, nower at which various nonlinear terms are large, $f \neq=0.5$, $r_{c}=100 \mu m, \Delta R=50 \mu m$, minimum resonance
absorption. 


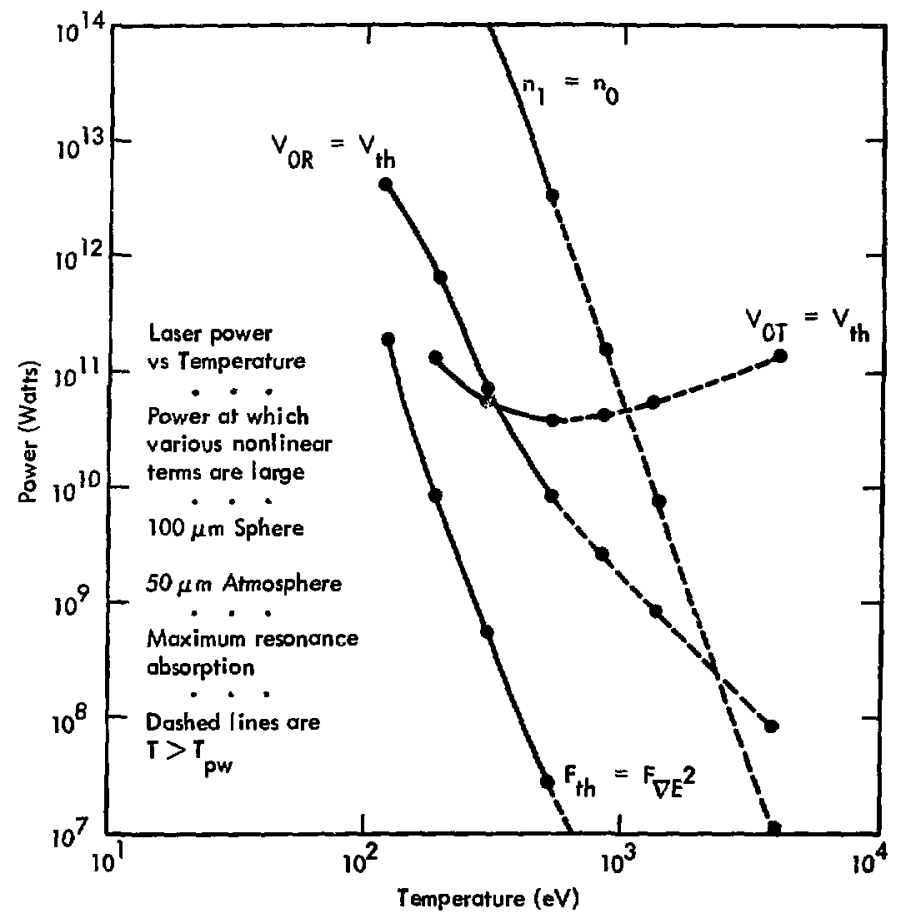

Figure IV.33 Laser power versus temperature, power at which various nonlinear terms are large, $f=0.5$. $r_{c}=100 \mu \mathrm{m}, A R=50 \mathrm{\mu ln}$, maximum resonance absorption. 


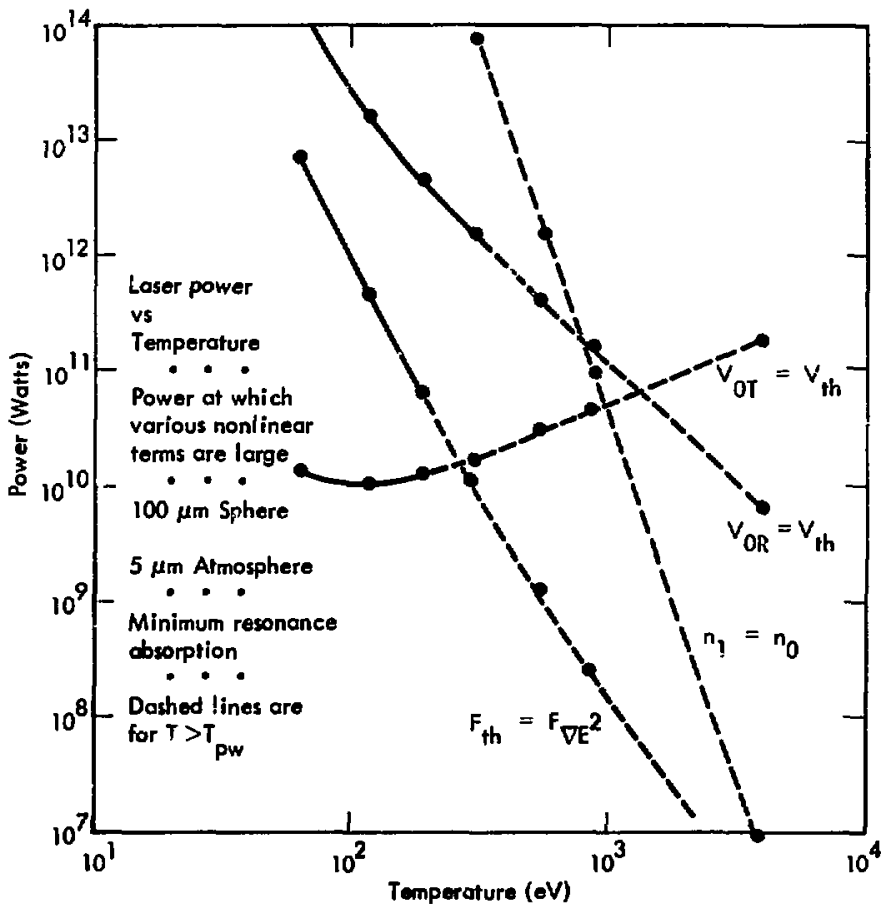

Figure IV.34 Laser power versus temperature, parer at which varfous nonlinear terms are large. $f=0.5$, $r_{\mathrm{c}}=100 \mathrm{um} . \Delta R=5 \mathrm{\mu m}$, minimum resonance absorption. 


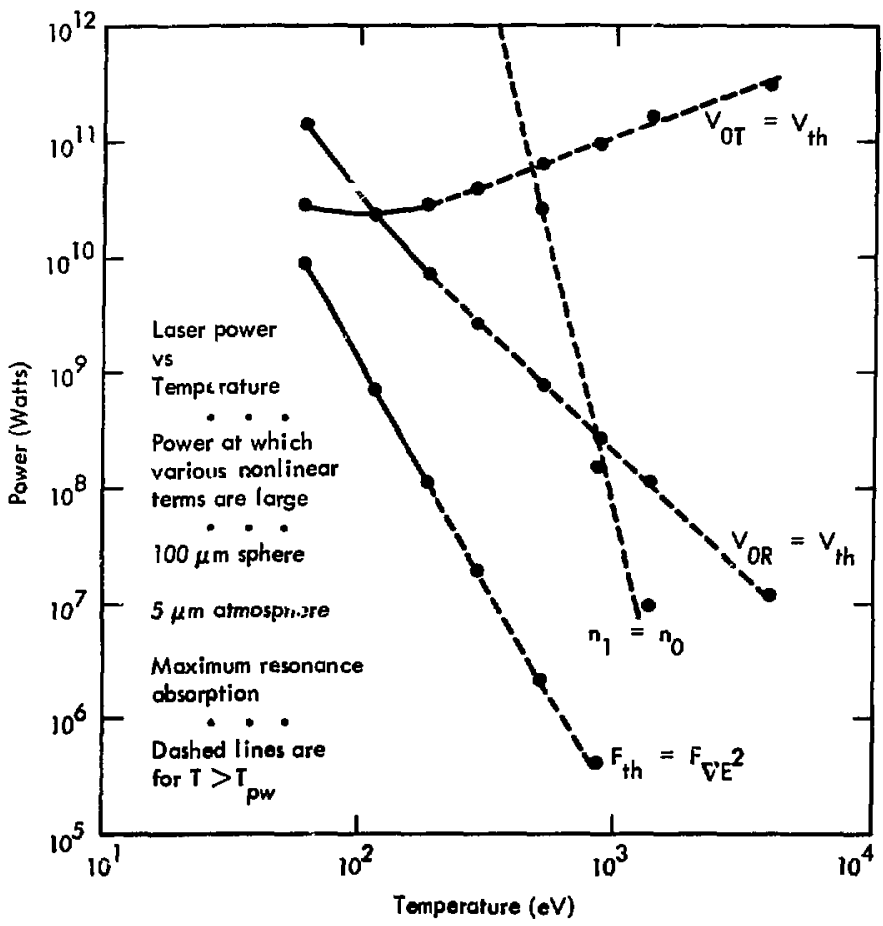

Figure IV.35 Laser power versus temperature, power at which various nonl inear terms are large, $f . \vec{*}=0.5$, $r_{c}=100 \mu \mathrm{m}, \Delta R=5 \mu \mathrm{m}$, maximum resonance absorption. 


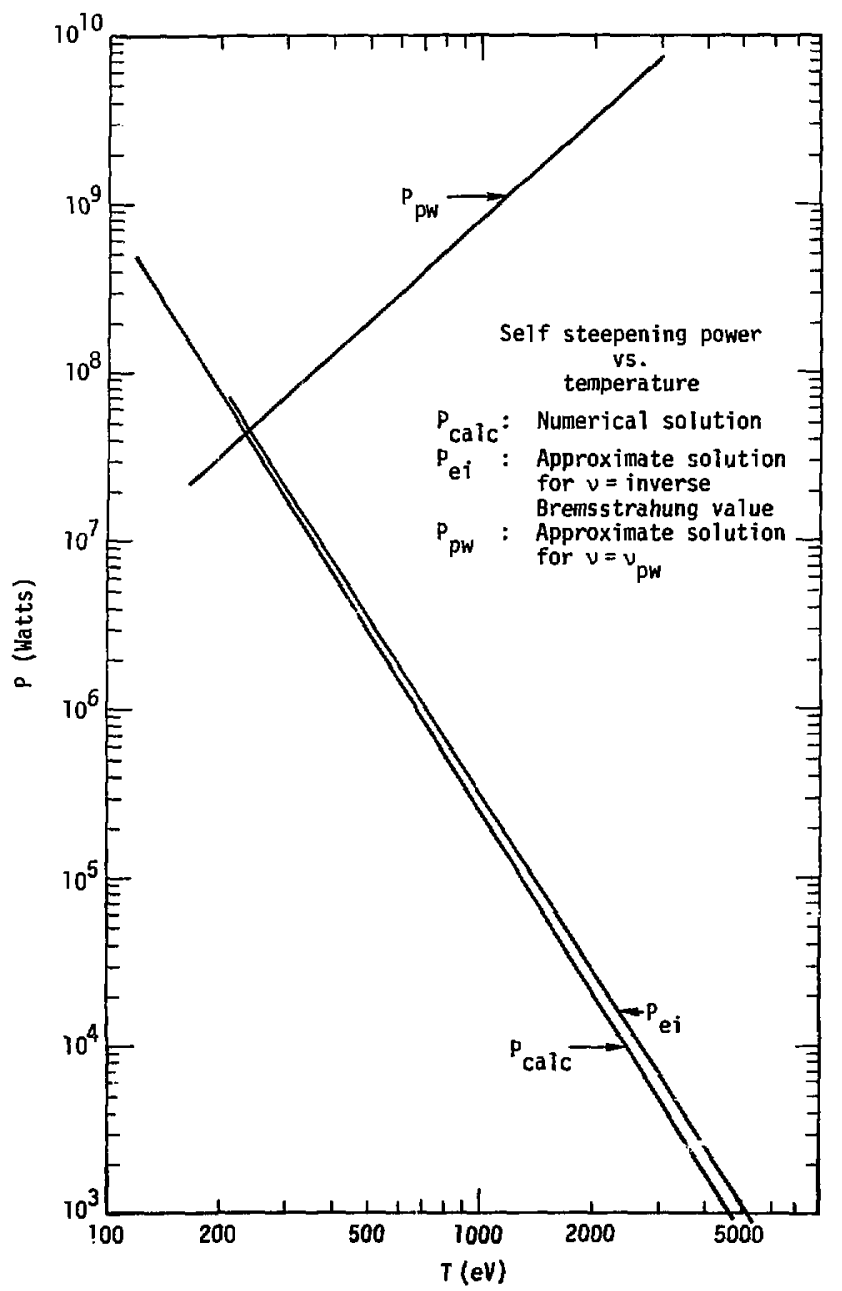

Figure IV.36 Self steepening power versus temperditure. 


\section{SUMMARY AND CONCLUSIONS}

We have developed and implemented a practical method for cslculating the effects of the interaction between a focused laser beam and a dense, spherically symmetric plasma. These calculations have been done for one micron light and linear density profiles in the plasma, with variable scale length, and for plasma radii of 100 micrometers. Data presented here serve mainly to demonstrate the type of results available, without addressing in detail any one specific problem in the laser-plasma coupling. The solutions here are oltained by solving the complete set of Maxwell's equations in steady state and therefore are an impro'ement over ray tracing solutions, which fail to correctly treat polarization effects, such as resonance absorption. Exitation of plasma waves by the longitudinal fields in the focal volume which are a result of vector diffraction (BOININ and HOLF (1965)) are also exactly treated. This method cannot be thought of as a replacenent for ray trace codes, however, because it cannot be applied to plasmas without spherical symmetry. In this chapter we will disruss the applications which this technique is suited to analyze, and discuss additional modifications which could improve its usefulness as a tool in studying loser-plasma interactions.

Afplications fall roughly into two categories and we can discuss these in terms of Figures IV.32 through IV.35. For low temperatures and low incident beain power the equations solved here accurately describe the physics of the interaction, limited only by the anset of 
hydradynamic effects which alter the spherical symmetry. For these power and temperature conditions, which basically insure that classical absorption dominate in the energy loss to the beam, this technique can be used to study details of the absorption such as the effect of varying density gradients and plasma sizes or to analyze alternate target designs. The solutions become inaccurate because of deviations from spherical symmetry which follow from hydrodynamic motion.

To attain the high compression needed for efficient energy production, laser fusion systems must employ multiple lasers or complex optics to provide nearly uniform irradiation of the plasma. (NUCKOLIS, W00D, THIESSEN and ZIMMERMAN (1972)). To model this configuration, one could couple one dimensional or multidimensional hydrodynamic codes to these electromagnetic solutions, because the plasma will remain spherically symmetric even as it moves. Under these circumstances, the curve described by $\mathrm{P}_{\mathrm{PE}}{ }^{2}$ in Figures IV.32 through IV.35 does not apply, and the powertemperature regime for which the theory developed here applies is greatly expanded. At higher temperatures, as resonance absorption beccmes important, the spherical symietry may be lost, but this can be monitered in the calculations of the density profile.

The first category for applying the techniques is in the powertemperature regime for which the physics is well approximated by the equations we solve; the second is the regime for which these approximations begin to break down. Here solutions given by the linearized 
steady state equations are useful as a means of estimating the non] inear terms to first order. Because the onset of the nonlinear effects occurs at the temperature for which resonance absorption begins to dominate the absorption, however, it is important that details of the plasma wave be accurately caiculated. In the method described in this report, plasma wave amplitudes are inaccurate for $T>T_{p w}$, because we have dropped the temperature dependent terms (except for the parameter $v(T)$ ) from Equations 3.3. The plasma wave at the critical surface, without the danping provided by these terms, will continue to grow as the temperature is increased. The most simple improvement that can be made is to modify the spatial dependence of the collision frequency so that, at the critical surface, the field components will be damped according to $\nu_{\mathrm{pw}}$ rather than $\nu$. This introduces an error, because oniy the electrostatic fields should be damped with the higher value, but for small $v$, the electromagnetic component of the field at $r_{c}$ is small compared to the electrostatic component anyway.

The ideal and most accurate : eatment is to solve Equations 3.3 without further approximation.

With these improvements, this technique (in some cases with modifications to include pressure terms as well) can be used to analyze the onset of nonlinear phenomena, such as spontaneous magnetic sield generation (THOMPSON, MAX and ESTABROOK (1975)) and filamentation (LANGDON and LASINSKI (1975) and VALEO and ESTABROOK (1975)) or hydrodynamic instabilities such as Rayleigh-Taylor (LINDL and MEAD (1975)). (We have not attempted to provide an exhaustive bibliography on these nonlinear effects in a laser irradiated plasma, and only mention 
recent work relating to laser fusion applications). In all cases the influence of the incident electromagnetic field in either driving the instabilities or in providing the perturbation to start them (through nonumiform heating or nonunformities in the beam itself, such as hot spots), can be analyzed.

In sumnary, we have described a technique by which the interaction of a focused laser beam with a dense, spherically symmetric plasma can be studied. We have analyzed the scattering and absorption of a laser beam as the laser and plasma parameters are adjusted and as the plasma is moved relative to the laser beam focus, and we have studied the spatial variation of the absorption and the standing electromagnetic waves. The limitations of the theory, as established by linearization and other approximations, have been estimated in a self-consistent fashion as a function of the plasma temperature and incident laser beam power, and we identify improvements which, if implemented, extend the range of validity of the solutions. Finally we discuss further applications of the existing technique and potential applications for modified versions which include hydrodynamic effects. 


\section{ACKNOWLEDGMENTS}

The assistance of many individuals was essential to the successful completion of the research reported in this thesis. Foremost among then is A. J. Glass, whose direction throtghout all phases of this project made this achievement possible. Also deserving special notice is $\mathrm{J}$. S. DeGroot, who has made numerous invaluable suggestions in the later stages of this efiort, particularly in the preparation of Chapter IV. I am pleased to acknowledge fruitful discussions concerning the techniques developed in Chapter III with J. T. Hunt and the assistance of R. F. Tooper in providing several key references. My gratitude is extended to the Fannie and John Hertz Foundation, which has supported my graduate study, to the staff of the Department of Applied Science, Day is, Livermore, for its continual support in administrative matters, and to the Lawrence Livermore Laboratory, for the use of facilities during this period of research. The difficult and exhausting task of typing the manuscript was expertly performed by B. B. Pierce. 


\section{APPENDIX I}

Diffraction of Light by Circular and Annular Openings

A standard problem treated in nearly all textbooks on optics and electromagnetic theory is the Fraunhofer diffraction cf piane electromagnetic waves by circular openings. With the procedure developed in Chapter [I, we can numerically solve for the Fraunhofer diffraction patterns of beams having axial symmetry, both circular and annular. We will make comparisons with exact solutions using a slightly modified version of the computer code used throughout this study, as a means of verifying the validity of the multipole expansion technique and the computer procedures implementing it.

The well known solution for the Fraunhofer diffraction pattern of the circular opening is

$$
\frac{I(r)}{I_{0}}=\left[2 \frac{d_{1}(r)}{r}\right]^{2}
$$

Where $I$ is the beam intensity and $J_{1}$ is a Bessel function of order 1. The annular opening gives the result

$$
\frac{I(r)}{I_{0}}=\frac{1}{\left(1-\varepsilon^{2}\right)^{2}}\left[\frac{2 J_{1}(r)}{r}-\varepsilon^{2}\left(2 \frac{J_{1}(r r)}{c r}\right)\right]^{2}
$$

in which the area which is being illuminated is specified by

$$
\varepsilon a \leqslant r \leqslant a
$$

and $a$ is the outer radius of the hole. 
The circular hole is the special case $\varepsilon=0$. (BORN and WOLF (1975), p. 417).

In Figure Al.1, we give results for comparison with those of Born and Wolf, for $\varepsilon=0 ., 0.5$, and 6.9 , where we approximate the plane wave by taking $N$ in equation 2.23.b to be 100. The annular structure is obtained by subtracting a second beam with a smaller $\theta_{\mathrm{m}}$ from the first prior to projection onto the spherical harmonics. 


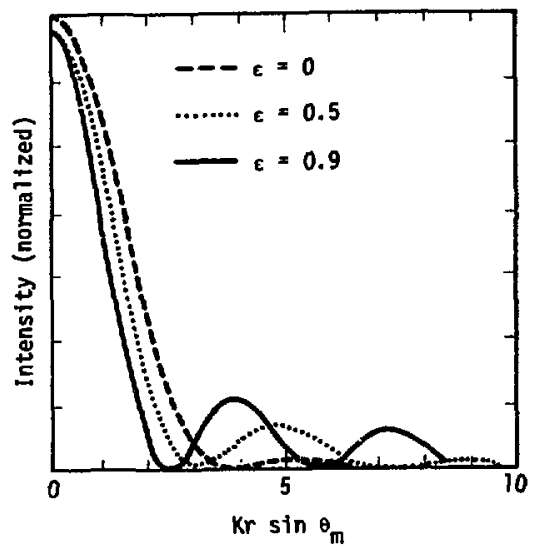

Figure Al.l Numerical solutions of the Fraunhofer diffraction patterns of a plane wave normally incident onto ctrcular and annular openings. 
APPEHDIX !I

Vector flelds in the focal volume of a Converging Lens

The vectoral nature of the diffraction pattem in the focal volume of a converging lens has been analyzed in a series of papers by MOLF (1959), RICLARDS and hOLF (1959), BOIVIM and HDLF (1965), and BOIVIH, OOH, and HOLF (1967). In these papers the diffracted fields are determined for a variety of parameters by the numerical evaluation of a one-dignensional integral for each point in the focal volume. We can also calculate the diffracted fields by specifying a plasma target with a negligible clectron density, in which case the fields are effectively in free soace. The orocedure for calculating the fields is entirely different from that of Wolf. hut exactly the same as we use when calculating the fields in the presence of alasma, and consequently, Wolf's results provide an excellent, source for determininn the accuracy of the implementation of the procedures described in Chapters II and II?. As Holf uses a square radial beam profile, we aporoximate the incident beam by using $N=100$, in equation 2.23.b.

He give the result for $\langle \$\rangle$, the time average Poynting vactor and for $\angle E:^{2}$, the electric energy density, in tho olanes $\phi=0$., and $\phi=90^{\circ}$ for an $f \neq 0.5$ focusing system, for comparison with results given in $B W$ and $B D W$, above.

Our plots differ slightly, as Holf uses the transverse $(v=$ $k r \sin \theta_{m}$ ) and longitudinal ( $u=k z \sin ^{2} 0_{m}$ ) scale factors, while our coordinates are simply $\mathrm{kr}$ and $\mathrm{kz}$. He have also compared neak and valley values with some of Holf's nublished results and find the agreement to be exact. 
Maximum value is at $Z=0, r=0$

Contour values diminish from maximum in steps of $50 \%$.

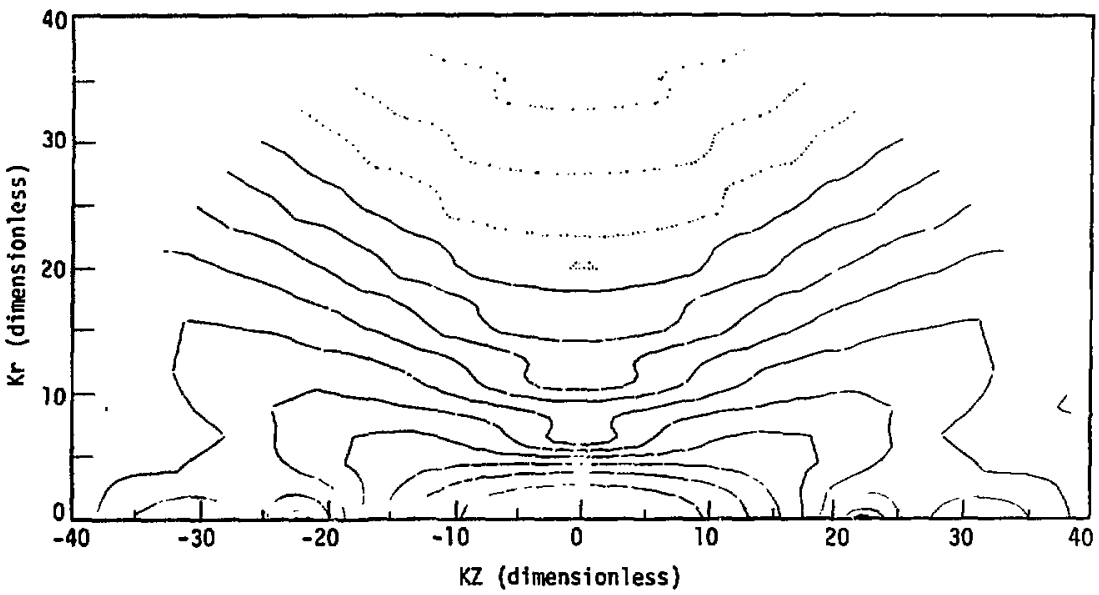

Figure A2.1 Electric energy density in focal volume, $\phi=0$. 
Maximun value is at $Z=0, r=0$

Contour values diminish from maxinum in

steps of $50 \%$.

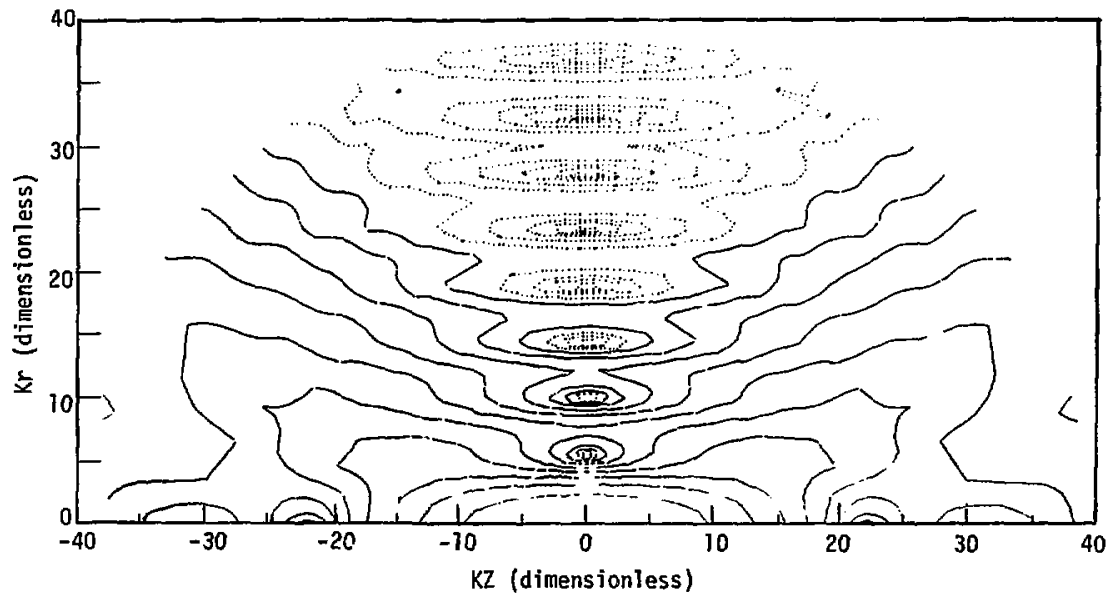

Figure A2.2 $|S|$, time ayeraged Poynting vector in focal 
Maximum value is at $Z=0, r=0$ Contour values diminish from maximum in steps of $50 \%$.

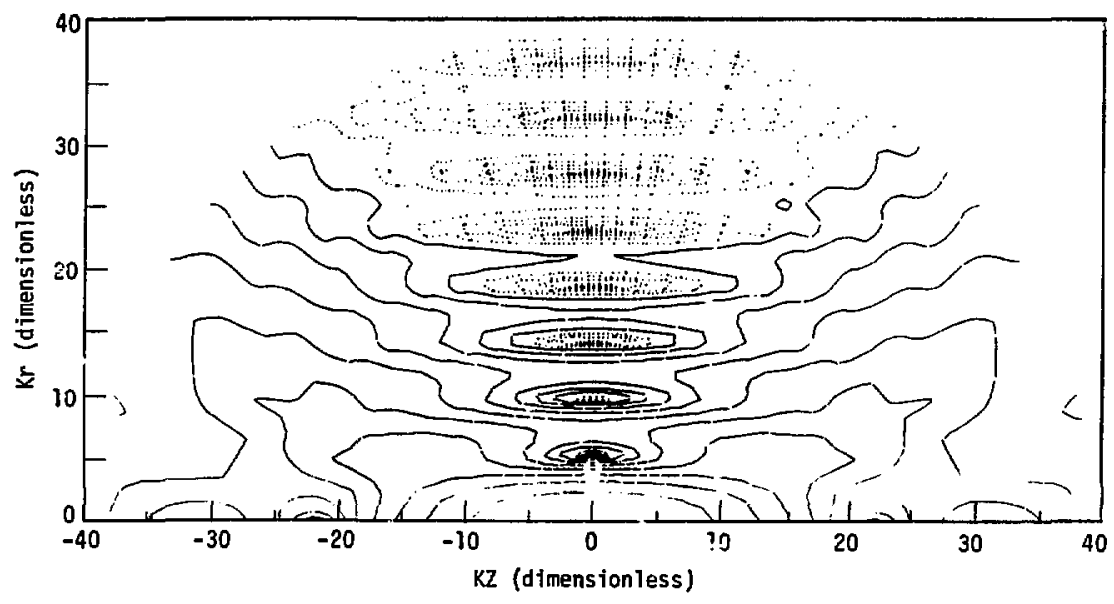

Figure A2.3 Electric energy density in focal volume, $\phi=90^{\circ}$. 
Maximum value is at $z=0, r=0$

Contour values diminish frcm rioximun in

steps of $50 \%$.

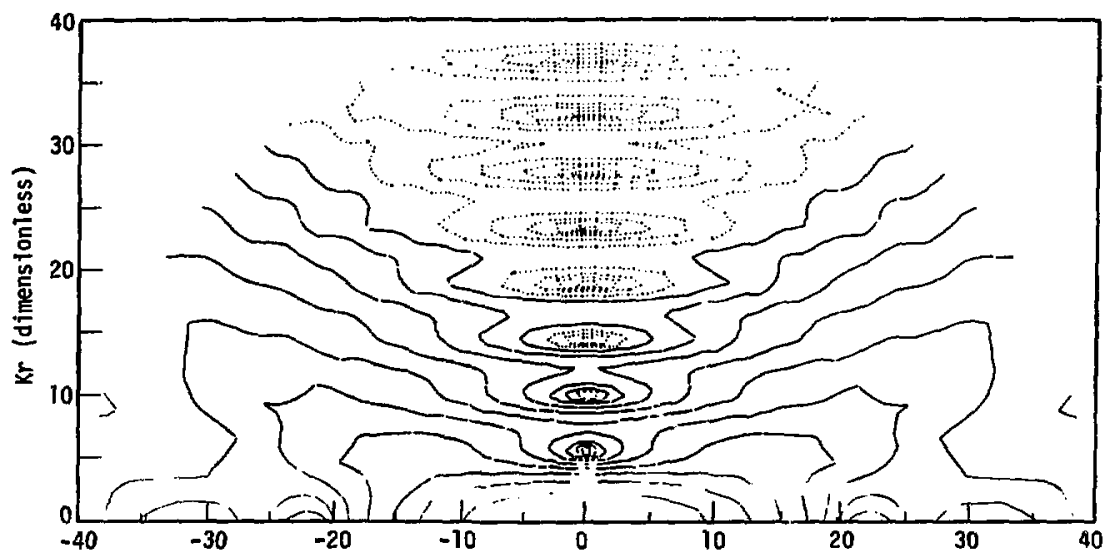

Figure A2.4 JS|, time averaged Poynting vector in focal volume, $\phi=90^{\circ}$. 


\section{APPENDIX III}

\section{Spherical Bessel Functions}

We have described in Chapter III a method for salving the waye eciuation in a spherically symetric medium, which, for $c=1$, has exact solutions consisting of the product of a spherical Bessel function and a spherical harmonic function. In Appendix 2 we showed that the free space solutions of the wave equation agreed with the RICHARIS and HOLF (1959) theory, when sunmed over the incident multipole spectrum. He will now look at the individual multipole solutions, to verify that in free space the radial functions $x_{Q}$ and $y_{2}$ are spherical Bessel functions. We use the same computational procedure when evaluating $x_{\ell}$ and $y_{\ell}$ in the plasma but here we take s to be unity. The quantities $\left|x_{Q}\right|^{2}$ and $\left|y_{\ell}\right|^{2}$ are plotted tor $\ell=1,2$, and 3 in Fig!? ${ }^{2}$ A3.1. $\left|x_{\ell}\right|^{2}$ and $\left|y_{\ell}\right|^{2}$ are identical to 4 significant figures. Referring to ABRAMOWITZ and STEGUN (1965), both $\left|x_{\ell}\right|^{2}$ and $\left|y_{l}\right|^{2}$ are identical to $\left|j_{\ell}\right|^{2}$, which we demonstrate explicitly by comparing the calculated zerops with tabulated values, in Table A3.1. The calculated zeroes are made to depart fron the correct values by enlarging the grid used in the calculations, although reasonably accurate zeroes are determined even at 10 grid points per free space wavelength. 
TABLE A 3.1

$$
x \text { position of } \pi^{\text {th }} \text { zero of } j_{l}
$$

l $\mathbf{m}$

$x$ (micrometers)

$$
\text { exact }
$$

$100 \quad 20 \quad 10 \quad 5$

(grid points per free space wavelength)

11.71515

$\begin{array}{llll}.7152 & .7160 & .7191 & .7328\end{array}$

$12 \quad 1.2295$

$\begin{array}{lll}1.230 & 1.230 \quad 1.231\end{array}$

$21 \quad .97728$

$.9173 \quad .9180$

.9188

$\begin{array}{lll}1.448 & 1.448 & 1.449\end{array}$

221.4475

$1.112 \quad 1.112 \quad 1.114$

311.1122

1.112

1.114 


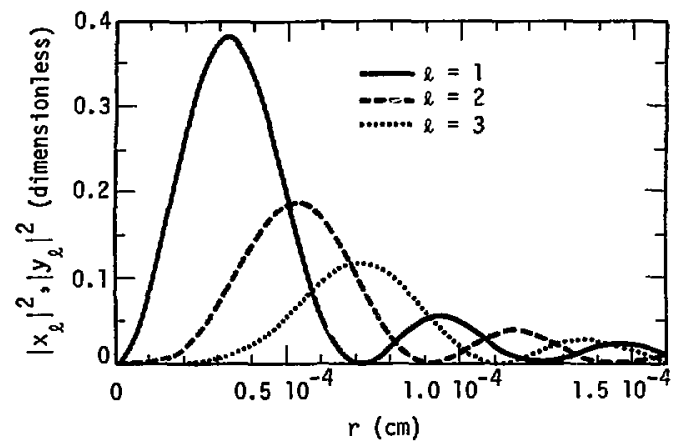

Figure A3.1 $\left|x_{\ell}\right|^{2}$ and $\left|y_{\ell}\right|^{2}$ for $E=1, \ell=1,2$, and 3 . 


\section{APPENOIX IV}

\section{Airy Function Solutions}

The radial component of the transverse electric mode satisfies the equation

$$
\frac{d^{2} r y_{\ell}}{d r^{2}}+\left[k^{2} \varepsilon-\frac{\ell(\ell+1)}{r^{2}}\right] r y_{\ell}=0
$$

which for $\varepsilon$ linearly dependent on $r$,

$$
\varepsilon=\frac{r-r c}{\Delta R}
$$

and using $w=r y_{\ell}$

gives

$$
\frac{d^{2} w}{d r^{2}}+\frac{k^{2}}{\Delta R}\left[r-r_{c}-\frac{\ell(\ell+1) \Delta R}{(k r)^{2}}\right] w=0 .
$$

Defining

$$
p=\left(\frac{k^{2}}{\Delta R}\right)^{1 / 3}\left[r-r_{c}-\frac{\ell(\ell+]) \Delta R}{(k r)^{2}}\right]
$$

we have

$$
\frac{d^{2} w}{d p^{2}}+p w \simeq 0
$$


when

$$
\Delta R \geqslant r_{c}, \ell \ll k r
$$

A4.6 has as solutions, the Airy functions

$$
w=A i(p) \text {. }
$$

Rather than compare our radial solutions with Airy functions, we will compare our results with those calculated by WHITE and CHEN (1974), p. 568, for the same parameters, in Figure A4.?.

Again we consider the effect of coarser grid spacing on the solutions, looking at $100,20,10$, and 5 grid points per free space wavelength. In this instance, the reduction of the number of grid points does not greatly affect the solution because automatic regridding is built into the computer procedure used in Chapter III, to insure that accurate solutions of the TM equation are maintained when marching through the singularity at the critical surface, and the identical grids are used for the TE solution here. 

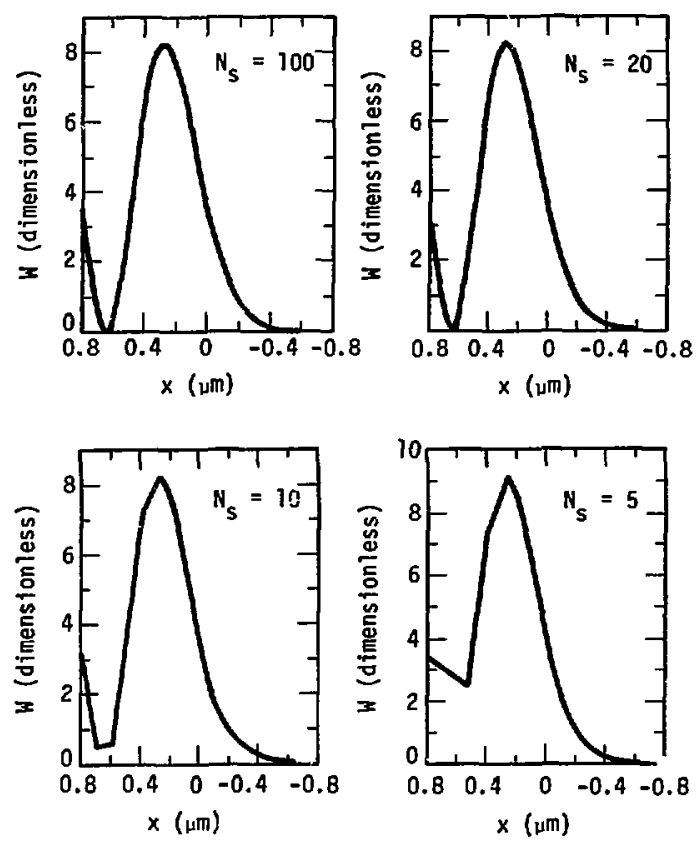

Figure A4.1 Numerical solution for comparison with Airy function. $N_{s}$ is number of grid points per freespace wavelength. 
APPENOIX $V$

Absorption With Linear Gradients

In Appendix IV we showed that for $\varepsilon$ linearly dependent on the radius, and when

$$
r_{c} \gg \frac{\ell(\ell+1) \Delta R}{(K r)^{2}}
$$

the product of the radius by the $r$ dependent component of the TE potential function satisfied the Airy equation, and we compared our solutions with comparable results from WHITE and CHEN (1974). Similarly, the $r$ dependent component of the $T H$ potential function satisfies the same equation as the transverse magnetic wave in Cartesian geometry, and we verify our computational procedure in this instance by calculating the resonance absorption as a function of $\ell$, and making plots to compare with the results of FORSELUND et al (1975). We find virtually exact agreement (Figure 45.1 ) when the condition A5.1 is satisfied. 


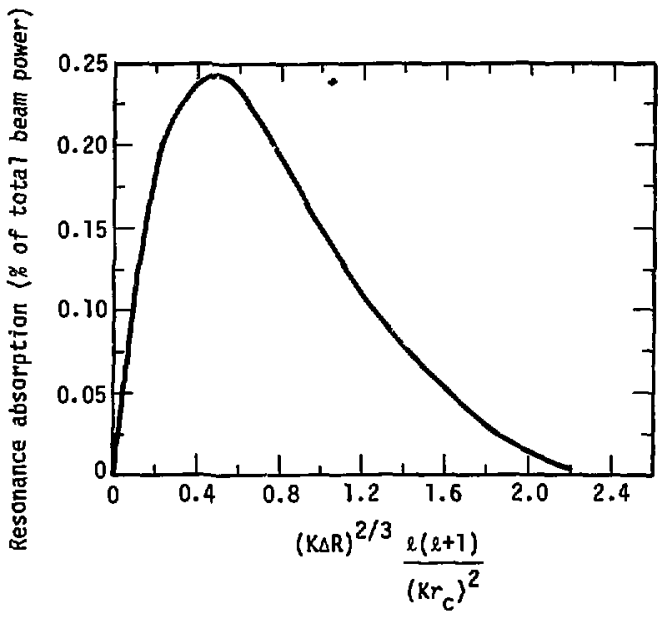

Figure A5.1 Resonance absorption in a medium with a linearly varying dielectric constant. 


\section{BIP'.IOGRAPHY}

Abramowitz, M. and J. Stegun, Handbook of Mathematical Functions

U.S. Government Printing Office, Washington DC, (1964)

Arnush, Donald, IEEE Trans. A.P. 12, 86 (1964)

Boivin, A., and E. Holf, , ilys. Rev, 138, 1561 (1965)

Boivin, A., J. Dow and E, Wolf, J. 0pt. Soc. Am. 57, 1171 (1967)

Born, M. and E. Holf, Principles of Optics (Pergamon Press, Inc. New York, 5th ed., 1975)

Budden, K. G., Radio Waves in the Ionosphere (Cambridge University Press, London, 1961)

Dawson, J., P. Kaw and B. Green, Phys. Fluids 12, 875 (1969)

DeGrsot, J. S. and J. E. Tu11, Phys. Fluids 18, 679 (1975)

Denisov, N. G., Soviet Phys. JETP 4, 544 (1957)

Forselund, D. H., J. M. Kindel, Kenneth Lee, E. L. Lindman and R. L. Morse, Phys. Rev. 11, 679 (1975)

Freidberg, J. P., R. H. Mitche11, R. L. Morse, and L. I. Rudsinski, Phys. Rev. Lett. 28, 795 (1972)

Ginzburg, V. L., The Propagation of Electromagnetic yaves in Plasmas, (Pergaman, New York, T964)

Glaze, J. A, D. R. Speck and J. T. Hunt, "Geometrical Theory of Nonlinear Phase Distortion of Intense Laser Beams", UCRL-76834 (1975) to be published

Jackson, J. D., Classical Electrodynamics (John Wiley \& Sons, Inc., NY, 1962)

Langdon, A. B. and B. F. Lasinski, nhys. Rev. Lett. 34, 934 (1975)

Lind1, J. D. and W. C. Mead, Phys. Rev. Lett. 34, 1273 (1975)

Luneburg, R. K., Mathematical Theory of Optics (University of California Press, Berkeley and Los Angeles, 1966)

Nuckolls, J., L. Wood, A. Thiessen and G. Zimmerman, Nature 239, 139 (1972) 
Nuckulls, John H., "Laser Induced Implosion and Thermonuclear Burn", in Laser Interaction and Related Plasma Phenomena, ed. Helmut $\mathrm{j}$. Schwarz and Heinrich Hora (P1enum Press, NY, 1974)

Piliya, A. D., Soviei Phys. Tech. Phys. 11, 609 (1966)

Richards, B. and E. Wolf, Proc. Roy. Soc. (London), 1253, 358 (1959)

Thompson, J. J., C. E. Max and K. G. Estabrook, Phys. Rev. Lett. 35, $663(1975)$

Valeo, E. J. and K. G. Estabrook, Phys. Rev. Lett. 34, 1008 (1975)

White, R. B. and F. F. Chen, Plasma Phys. 16, 505 (1974)

Wolf, E., Proc. Roy. Soc. (London), A253, 349 (1959)

Wyatt, P.J. Phys. Rev. 127, 1837 (1962) 